\title{
Generic Metrics, Irreducible Rank-One PU(2) Monopoles, and Transversality
}

\author{
PAUl M.N. FEeHAN ${ }^{1}$
}

\section{Introduction.}

Our main purpose in this article is to prove that the moduli space of solutions to the $\mathrm{PU}(2)$ monopole equations is a smooth manifold of the expected dimension for simple, generic parameters such as (and including) the Riemannian metric on the given four-manifold: see Theorem 1.3 in $\S 1.2$. In [16] we proved transversality using an extension of the holonomy-perturbation methods of Donaldson, Floer, and Taubes [12], [13], [22], [68], together with the existence of an Uhlenbeck compactification for the perturbed moduli space. In [18], [19] we discuss applications of these results to the PU(2) monopole program for proving the equivalence between Donaldson and Seiberg-Witten invariants conjectured in [74], [47] (see, for example, [18], [52], [53], [54], [56], [59]). However, it is an important and interesting question to see whether there are simpler alternatives to the holonomy perturbations and this is the issue we address here.

The idea that one should be able to use $\mathrm{PU}(2)$ monopoles to prove Witten's conjecture concerning the relation between the two types of fourmanifold invariants was proposed by Pidstrigach and Tyurin in 1994; see [16], [19], [18], [52], [53], [54], [56], [59], [71] for work on this program due independently to the author and Leness, Pidstrigach and Tyurin, and Okonek and Teleman. The idea itself can be informally described quite quickly, the key point being that the moduli space of $\mathrm{PU}(2)$ monopoles contains the moduli space of anti-self-dual connections together with copies of the various Seiberg-Witten moduli spaces, these forming singular loci in the higher-dimensional moduli space of $\mathrm{PU}(2)$ monopoles. In principle, then, one should be able to use intersection theory on this higher-dimensional moduli space to relate the two kinds of invariants. In practice, despite the

\footnotetext{
${ }^{1}$ Supported in part by an NSF Mathematical Sciences Postdoctoral Fellowship under grant DMS 9306061 and by NSF grant DMS-9704174
} 
simplicity of this basic idea, the difficulties surrounding its implementation are daunting. For this program to succeed one needs to know that the moduli space of $\mathrm{PU}(2)$ monopoles - away from the anti-self-dual and SeibergWitten points - is a smooth manifold of the expected dimension. This ensures that these exceptional points are the only singularities and that the $\mathrm{PU}(2)$ monopole moduli space forms a smooth (though non-compact, because of bubbling) cobordism between the links of the singularities.

For PU(2) monopoles, we would ideally like an analogue of the FreedUhlenbeck generic metrics theorem for the anti-self-dual equation [15], [23] or the generic parameter result introduced by Witten for the Seiberg-Witten equations [37], [74]. However, results of this kind for the PU(2) monopole equations appear to be much harder to prove. One of the outcomes of our joint work with Leness [16] was a proof that one could nonetheless obtain a useful transversality result via holonomy perturbations by extending related ideas of Donaldson and Taubes [12], [13], [68]. Such holonomy perturbations are important when considering three-manifold versions of the monopole equations. While all of the intersection theory calculations on the moduli space of $\mathrm{PU}(2)$ monopoles described in [19] could be carried out using holonomy perturbations, the generic-parameter result (Theorem 1.3) established in the present article represents a very significant simplification and has been the basis of our continuing work on the project to mathematically verify Witten's conjecture [19], [20], [21]. Our generic-parameter approach (see $\S 1.3$ for an outline) makes essential use of certain unique continuation properties for reducible solutions to the $\mathrm{PU}(2)$ monopole equations which we developed in our earlier work [16], although not the holonomy perturbations themselves.

Issues of transversality also appear to play a significant role in the ongoing work of Kronheimer and Mrowka to complete a three-dimensional analogue of the Pidstrigach-Tyurin program and use $\mathrm{PU}(2)$ monopoles to prove "Property P" for knots, via a comparison of Yang-Mills and Seiberg-Witten Floer homologies [36], [40]. Moving outside the realm of low-dimensional manifolds per se, a technical issue which plagued the definition of GromovWitten invariants for general symplectic manifolds concerned the absence of generic-parameter transversality results for boundary components of the compactification. For semi-positive symplectic manifolds such transversality difficulties do not arise [61]. The problem was eventually addressed via an important differential-geometric extension of certain algebraic excess intersection theory methods - by Fukaya and Ono [25], Li and Tian [43], Liu and Tian [44], Ruan [60], and Siebert [64], using a variety of different approaches. A solution was also announced by Hofer and Salamon. In the case 
of $\mathrm{PU}(2)$ monopoles it is already a difficult problem to obtain transversality away from the exceptional solutions, even when no bubbling has occurred. In general, it is not possible to ensure that the loci of exceptional solutions are unobstructed, so we must still use differential-geometric excess intersection theory techniques [19] broadly similar to those of [43], [60] and going back to ideas of [6], [13], [15], [49], [66].

\subsection{PU(2) monopoles.}

Throughout this article, $X$ denotes a closed, connected, oriented, smooth four-manifold. In order to state our results, we briefly recall the description of the moduli space of $\mathrm{PU}(2)$ monopoles from [16], [18]. We give $X$ a Riemannian metric and consider Hermitian two-plane bundles $E$ over $X$ whose determinant line bundles det $E$ are isomorphic to a fixed Hermitian line bundle endowed with a fixed $C^{\infty}$, unitary connection $A_{e}$. Let $\left(\rho, W^{+}, W^{-}\right)$be a $\operatorname{spin}^{c}$ structure on $X$, where $\rho: T^{*} X \rightarrow \operatorname{Hom}_{\mathbb{C}}\left(W^{+}, W^{-}\right)$is the Clifford map, and the Hermitian four-plane bundle $W:=W^{+} \oplus W^{-}$is endowed with a $C^{\infty}$, unitary connection. The unitary connection on $W$ uniquely determines a Riemannian connection on $T^{*} X$, via the Clifford map $\rho$, and a unitary connection on $\operatorname{det} W^{+}$; conversely, a choice of Riemannian connection on $T^{*} X$ and unitary connection on $\operatorname{det} W^{+}$induce a unitary connection on $W$. The connection on $W$ is called $\operatorname{spin}^{c}$ if it induces the Levi-Civita connection on $T^{*} X$ for the given Riemannian metric.

Let $k \geq 2$ be an integer and let $\mathcal{A}_{E}$ be the space of $L_{k}^{2}$ connections $A$ on the $\mathrm{U}(2)$ bundle $E$ all inducing the fixed determinant connection $A_{e}$ on $\operatorname{det} E$. Equivalently, following [38, $\S 2(\mathrm{i})]$, we may view $\mathcal{A}_{E}$ as the space of $L_{k}^{2}$ connections $A$ on the $\mathrm{PU}(2)=\mathrm{SO}(3)$ bundle $\mathfrak{s u}(E)$. We shall pass back and forth between these viewpoints, via the fixed connection on $\operatorname{det} E$, relying on the context to make the distinction clear. Given a unitary connection $A$ on $E$ with curvature $F_{A} \in L_{k-1}^{2}\left(\Lambda^{2} \otimes \mathfrak{u}(E)\right)$, then $\left(F_{A}^{+}\right)_{0} \in L_{k-1}^{2}\left(\Lambda^{+} \otimes \mathfrak{s u}(E)\right)$ denotes the traceless part of its self-dual component. Equivalently, if $A$ is a connection on $\mathfrak{s u}(E)$ with curvature $F_{A} \in L_{k-1}^{2}\left(\Lambda^{2} \otimes \mathfrak{s o}(\mathfrak{s u}(E))\right)$, then $\operatorname{ad}^{-1}\left(F_{A}^{+}\right) \in L_{k-1}^{2}\left(\Lambda^{+} \otimes \mathfrak{s u}(E)\right)$ is its self-dual component, viewed as a section of $\Lambda^{+} \otimes \mathfrak{s u}(E)$ via the isomorphism ad $: \mathfrak{s u}(E) \rightarrow \mathfrak{s o}(\mathfrak{s u}(E))$. When no confusion can arise, the isomorphism ad $: \mathfrak{s u}(E) \rightarrow \mathfrak{s o}(\mathfrak{s u}(E))$ will be implicit and so we regard $F_{A}$ as a section of $\Lambda^{+} \otimes \mathfrak{s u}(E)$ when $A$ is a connection on $\mathfrak{s u}(E)$.

For an $L_{k}^{2}$ section $\Phi$ of $W^{+} \otimes E$, let $\Phi^{*}$ be its pointwise Hermitian dual and let $\left(\Phi \otimes \Phi^{*}\right)_{00}$ be the component of the Hermitian endomorphism $\Phi \otimes \Phi^{*}$ of 
$W^{+} \otimes E$ which lies in $\mathfrak{s u}\left(W^{+}\right) \otimes \mathfrak{s u}(E)$. The Clifford multiplication $\rho$ defines an isomorphism $\rho: \Lambda^{+} \rightarrow \mathfrak{s u}\left(W^{+}\right)$and thus an isomorphism $\rho=\rho \otimes \operatorname{id}_{\mathfrak{s u}(E)}$ of $\Lambda^{+} \otimes \mathfrak{s u}(E)$ with $\mathfrak{s u}\left(W^{+}\right) \otimes \mathfrak{s u}(E)$.

Let $\mathcal{G}_{E}$ be the Hilbert Lie group of $L_{k+1}^{2}$ unitary gauge transformations of $E$ with determinant one. It is often convenient to take quotients by a slightly larger symmetry group than $\mathcal{G}_{E}$ when discussing pairs, so let $S_{Z}^{1}$ denote the center of $\mathrm{U}(2)$ and set ${ }^{\circ} \mathcal{G}_{E}:=S_{Z}^{1} \times_{\left\{ \pm \mathrm{id}_{E}\right\}} \mathcal{G}_{E}$, which we may view as the group of $L_{k+1}^{2}$ unitary gauge transformations of $E$ with constant determinant. The stabilizer of a unitary connection on $E$ in ${ }^{\circ} \mathcal{G}_{E}$ always contains the center $S_{Z}^{1} \subset \mathrm{U}(2)$.

We call an $L_{k}^{2}$ pair $(A, \Phi)$ in the pre-configuration space,

$$
\tilde{\mathcal{C}}_{W, E}:=\mathcal{A}_{E} \times L_{k}^{2}\left(W^{+} \otimes E\right),
$$

a $\mathrm{PU}(2)$ monopole if $\mathfrak{S}(A, \Phi)=0$, where the ${ }^{\circ} \mathcal{G}_{E^{-}}$-equivariant map $\mathfrak{S}$ : $\tilde{\mathcal{C}}_{W, E} \rightarrow L_{k}^{2}\left(\Lambda^{+} \otimes \mathfrak{s u}(E)\right) \oplus L_{k}^{2}\left(W^{-} \otimes E\right)$ is defined by

$$
\mathfrak{S}(A, \Phi):=\left(\begin{array}{c}
F_{A}^{+}-\tau \rho^{-1}\left(\Phi \otimes \Phi^{*}\right)_{00} \\
\mathcal{D}_{A} \Phi+\rho(\vartheta) \Phi
\end{array}\right)
$$

where $\mathcal{D}_{A}: L_{k}^{2}\left(W^{+} \otimes E\right) \rightarrow L_{k-1}^{2}\left(W^{-} \otimes E\right)$ is the Dirac operator, while $\tau \in C^{\infty}\left(\operatorname{GL}\left(\Lambda^{+}\right)\right)$and $\vartheta \in \Omega^{1}(X, \mathbb{C})$ are perturbation parameters. We let $M_{W, E}:=\mathfrak{S}^{-1}(0)$ be the moduli space of solutions cut out of the configuration space,

$$
\mathcal{C}_{W, E}:=\tilde{\mathcal{C}}_{W, E} /{ }^{\circ} \mathcal{G}_{E}
$$

by the section (1.1), where $u \in{ }^{\circ} \mathcal{G}_{E}$ acts by $u(A, \Phi):=\left(u_{*} A, u \Phi\right)$.

As customary, we say that an $\mathrm{SO}(3)$ connection $A$ on $\mathfrak{s u}(E)$ is irreducible if its stabilizer in $\mathcal{G}_{E}$ is $\left\{ \pm \operatorname{id}_{E}\right\}$, corresponding to the center of $\mathrm{SU}(2)$, and reducible otherwise; we say that a pair $(A, \Phi)$ on $\left(\mathfrak{s u}(E), W^{+} \otimes E\right)$ is irreducible (respectively, reducible) if the connection $A$ is irreducible (respectively, reducible). We let $\mathcal{C}_{W, E}^{*} \subset \mathcal{C}_{W, E}$ be the open subspace of gauge-equivalence classes of irreducible pairs. We say that a section $\Phi$ of $W^{+} \otimes E$ has rank $r$ if, when considered as a section of $\operatorname{Hom}_{\mathbb{C}}\left(W^{+, *}, E\right)$, the section $\Phi(x)$ has complex rank less than or equal to $r$ at every point $x \in X$, with equality at some point; we say that a pair $(A, \Phi)$ on $\left(\mathfrak{s u}(E), W^{+} \otimes E\right)$ has $\operatorname{rank} r$ if the section $\Phi$ has rank $r$; if $(A, \Phi)$ has rank zero, that is $\Phi \equiv 0$ on $X$, we call $(A, \Phi)$ a zero-section pair. We let $\mathcal{C}_{W, E}^{0} \subset \mathcal{C}_{W, E}$ be the open subspace of gauge-equivalence classes of non-zero-section pairs and recall that $\mathcal{C}_{W, E}^{*, 0}:=\mathcal{C}_{W, E}^{*} \cap \mathcal{C}_{W, E}^{0}$ is a Hausdorff, Hilbert manifold [16, Proposition 2.8] represented by pairs with stabilizer $\left\{\operatorname{id}_{E}\right\}$ in ${ }^{\circ} \mathcal{G}_{E}$. Let $M_{W, E}^{*, 0}=M_{W, E} \cap \mathcal{C}_{W, E}^{*, 0}$ 
be the open subspace of the moduli space $M_{W, E}$ represented by irreducible, non-zero-section $\mathrm{PU}(2)$ monopoles.

If $(A, \Phi)$ is a $\mathrm{PU}(2)$ monopole then (see [16, Lemma 5.21])

$(A, \Phi)$ reducible $\Rightarrow(A, \Phi)$ has rank less than or equal to one.

However, it is an important observation, due to Teleman [54], [71], that

$$
(A, \Phi) \text { reducible } \nLeftarrow(A, \Phi) \text { rank one. }
$$

Indeed, counterexamples are easily constructed (at least for the unperturbed $\mathrm{PU}(2)$ monopole equations) when $X$ is a Kähler manifold with its canonical $\operatorname{spin}^{c}$ structure [54], [71] (see Appendix A.1). That is, a reducible PU(2) monopole necessarily has rank less than or equal to one but in general, there exist irreducible, rank-one $\mathrm{PU}(2)$ monopoles. If $\Phi \equiv 0$, then the $\mathrm{PU}(2)$ monopole equations (1.1) just imply that $A$ is an anti-self-dual connection on $\mathfrak{s u}(E)$; the locus of reducible PU(2) monopoles in $M_{W, E}$ can be identified with a union of Seiberg-Witten moduli spaces.

As we shall see in $\S 2$ it is not too difficult to prove that $M_{W, E}^{*, 0}$ is a smooth manifold of the expected dimension away from the locus of irreducible, rankone solutions using the perturbation parameters $(\tau, \vartheta)$ alone. However, as irreducible, rank-one solutions to (1.1) may be present in $M_{W, E}^{*, 0}$, it seems impossible to prove that the entire space $M_{W, E}^{*, 0}$ is a smooth manifold of the expected dimension using these parameters alone. A similar problem arises in the proof of transversality for the ' $\operatorname{spin}^{c}-\mathrm{ASD}^{-}$equations given in [58, Proposition I.3.5]; a version of these equations can be obtained from the equations (1.1) by omitting the quadratic term $\tau \rho^{-1}\left(\Phi \otimes \Phi^{*}\right)_{00}$. In the proof of [58, Proposition I.3.5] it is claimed that if $D_{A} \Phi=0$ and $\Phi$ is rank one, then $A$ is reducible [p. 277]: Teleman's counterexample shows that this claim is incorrect and he points out an error in their argument [71].

On the other hand, the fact that the counterexamples of [71] occur when $X$ is a complex, Kähler surface suggests that irreducible, rank-one solutions might not be present in the moduli spaces $M_{W, E}^{*, 0}$ for generic Riemannian metrics and compatible Clifford maps, so this is a feature of the equations (1.1) which we shall explore further in this article.

\subsection{Statement of results.}

We can now state our Uhlenbeck compactness and transversality results for the moduli space of $\mathrm{PU}(2)$ monopoles with the perturbations discussed in the preceding section. As we remarked earlier, applications of these results 
to the $\mathrm{PU}(2)$ monopole program for proving the equivalence of Donaldson and Seiberg-Witten invariants are described in [18], [19].

Theorem 1.1. Let $X$ be a closed, oriented, smooth four-manifold with $C^{\infty}$ Riemannian metric, spin structure $\left(\rho, W^{+}, W^{-}\right)$with spin ${ }^{c}$ connection on $W=W^{+} \oplus W^{-}$, and a Hermitian two-plane bundle $E$ with unitary connection on $\operatorname{det} E$. Then there is a positive integer $N_{p}$, depending at most on the curvatures of the fixed connections on $W$ and $\operatorname{det} E$ together with $c_{2}(E)$, such that for all $N \geq N_{p}$ the topological space $\bar{M}_{W, E}$ is second countable, Hausdorff, compact, and given by the closure of $M_{W, E}$ in $\cup_{\ell=0}^{N}\left(M_{W, E_{-\ell}} \times \operatorname{Sym}^{\ell}(X)\right.$ with respect to the Uhlenbeck topology, where $E_{-\ell}$ is a Hermitian two-plane bundle over $X$ with $\operatorname{det} E_{-\ell}=\operatorname{det} E$ and $c_{2}\left(E_{-\ell}\right)=c_{2}(E)-\ell$ for each integer $\ell \geq 0$.

Theorem 1.1 is simply a special case of the more general result proved in [16] for the moduli space of solutions to the perturbed PU(2) monopole equations in the presence of holonomy perturbations, so no separate proof is required. We include the statement here since it is more accessible in the absence of holonomy perturbations and because we appeal to it in [19], [20], [21].

Remark 1.2. The existence of an Uhlenbeck compactification for the moduli space of solutions to the unperturbed $\mathrm{PU}(2)$ monopole equations (1.1) was announced by Pidstrigach [56] and an argument was outlined in [59]. A. similar argument for the equations (1.1) (without perturbations) was outlined by Okonek and Teleman in [53]. An independent proof of Uhlenbeck compactness for (1.1) and other perturbations of these equations is also given in [71].

The perturbation parameters $(\tau, \vartheta)$ occurring in the statement of: Theorem 1.3 below appear explicitly in the $\mathrm{PU}(2)$ monopole equations (1.1) and are described further in $\S 2.1$. The perturbation parameter $f \in C^{\infty}\left(\mathrm{GL}\left(T^{*} X\right)\right)$ is a variation of the Clifford map $\rho: T^{*} X \rightarrow$ $\operatorname{Hom}_{\mathbb{C}}\left(W^{+}, W^{-}\right)$and of the Riemannian metric $g$ on $T^{*} X$ by an automorphism of $T^{*} X$ and is described further in $\S 3$; the perturbed PU(2) monopole equations with the three perturbation parameters $(f, \tau, \vartheta)$ are given in equation (3.12). Let $M_{E}^{\text {asd }}$ denote the moduli space of anti-self-dual $\mathrm{SO}(3)$ connections on $\mathfrak{s u}(E)$.

Theorem 1.3. Let $X$ be a closed, oriented, smooth four-manifold with $C^{\infty}$ Riemannian metric $g$, spin ${ }^{c}$ structure $\left(\rho, W^{+}, W^{-}\right)$with unitary connec- 
tion on $\operatorname{det} W^{+}$, and a Hermitian line bundle $\operatorname{det} E$ with unitary connection. Then there is a first-category subset $\mathcal{P}_{\mathrm{fc}}^{\infty}$ of the Fréchet space $\mathcal{P}^{\infty}$ of $C^{\infty}$ perturbation parameters $(f, \tau, \vartheta)$ such that for all $(f, \tau, \vartheta)$ in $\mathcal{P}^{\infty}-\mathcal{P}_{\mathrm{fc}}^{\infty}$ the following holds: For each parameter $(f, \tau, \vartheta)$ in $\mathcal{P}^{\infty}-\mathcal{P}_{\mathrm{fc}}^{\infty}$ and Hermitian two-plane bundle $E$ over $X$, the moduli space $M_{W, E}^{*, 0}(f, \tau, \vartheta)$ of $\mathrm{PU}(2)$ monopoles is a smooth manifold of the expected dimension,

$$
\begin{aligned}
\operatorname{dim} M_{W, E}^{*, 0}(f, \tau, \vartheta)= & \operatorname{dim} M_{E}^{*, \text { asd }}+2 \operatorname{ind}_{\mathbb{C}} \mathcal{D}_{A}-1 \\
= & -2 p_{1}(\mathfrak{s u}(E))-\frac{3}{2}(e(X)+\sigma(X)) \\
& +\frac{1}{2} p_{1}(\mathfrak{s u}(E))+\frac{1}{2}\left(\left(c_{1}\left(W^{+}\right)+c_{1}(E)\right)^{2}-\sigma(X)\right)-1 .
\end{aligned}
$$

As explained further in $\S 3.1$, the Levi-Civita connection on $T^{*} X$ for the metric $f^{*} g$, Clifford map $\rho \circ f$, and unitary connection on $\operatorname{det} W^{+}$define a $\operatorname{spin}^{c}$ connection on $W$. The rest of our article is devoted to proving Theorem 1.3.

\subsection{Outline of the proof and of the remainder of the article.}

We prove Theorem 1.3 in two steps, both of which have analogues in the case of the moduli space of anti-self-dual $\mathrm{SO}(3)$ connections over a four-manifold $X$ with $b^{+}(X)>0$ (although that constraint is not required here):

1. For generic parameters $(\tau, \vartheta)$ (and any parameter $f$ ), we show that the moduli space $M_{W, E}^{*, \mathrm{~h}}(f, \tau, \vartheta)$ of $\mathrm{PU}(2)$ monopoles $(A, \Phi)$ with $A$ irreducible and $\Phi$ rank two is a smooth manifold of the expected dimension.

2. For generic parameters $(f, \vartheta)$ (and any parameter $\tau$ ), we show that the moduli space $M_{W, E}^{*, 0}(f, \tau, \vartheta)$ of PU(2) monopoles $(A, \Phi)$ with $A$ irreducible and $\Phi \not \equiv 0$ contains no pairs $(A, \Phi)$ with $\Phi$ having rank one, that is, $M_{W, E}^{*, 0}(f, \tau, \vartheta)=M_{W, E}^{*, h}(f, \tau, \vartheta)$.

The first result, Theorem 2.2, is proved in $\S 2$ using the Sard-Smale theorem [65] and Aronszajn's unique continuation theorem [5], together with some linear algebra calculations reminiscent of those arising in the proof of the Freed-Uhlenbeck generic metrics theorem [23]. The second result, Theorem 4.1 , is considerably more difficult and is proved in $\S 4$ using an infinitedimensional analogue, for the family of perturbed Dirac operators $\mathcal{D}_{A, f, \vartheta}$, of the well-known 'period map' argument for the family of operators $d^{+, g}[11$, $\S \mathrm{VI}]$, [15, Corollary 4.3.15]; see also [23, Corollary 3.21], [67, Appendix B]. Combining these two results yields Theorem 1.3. In the case of the moduli 
space of $\mathrm{SO}(3)$ anti-self-dual connections, the first step is accomplished by the Freed-Uhlenbeck generic metrics theorem: that is, for generic metrics, $M_{E}^{\text {asd,* }}(g)$ is a smooth manifold of the expected dimension [15, Corollary 4.3.18], [23, Theorem 3.17]. The second step is achieved by the (finitedimensional) period-map argument: for generic metrics and $b^{+}(X)>0$, the moduli space $M_{E}^{\text {asd }}(g)$ contains no reducible connections [11, $\left.\S \mathrm{VI}\right],[15$, Corollary 4.3.15].

The detailed proof of Theorem 1.3 is, of course, more complicated than the preceding synopsis can convey, so we indicate how the remainder of this article is organized. Section 2 contains the proof that the moduli space of irreducible, rank-two PU(2) monopoles is regular. In $\S 2.1$ we define a parametrized moduli space of $\mathrm{PU}(2)$ monopoles and, assuming this is regular away from the loci of reducible or lower-rank $\mathrm{PU}(2)$ monopoles, we use the Sard-Smale theorem to show that the individual moduli spaces of irreducible, rank-two $\mathrm{PU}(2)$ monopoles are regular for generic parameters. The heart of the first step, then, is given in $\S 2.2$, where we show that the parametrized moduli space of irreducible, rank-two $\mathrm{PU}(2)$ monopoles is regular. Section 3 describes the perturbations of the Dirac operator: in $\S 3.1 \mathrm{we}$ compute the differential of the resulting family of Dirac operators and in $\S 3.2$ we define the $\mathrm{PU}(2)$ monopole equations and associated parametrized moduli space with the full set of perturbations. Section 4 contains the proof that, for generic parameters, the moduli spaces of irreducible $\mathrm{PU}(2)$ monopoles contain no rank-one pairs. Sections 4.1, 4.2, and 4.3 describe the loci of irreducible, rank-one $\mathrm{PU}(2)$ monopoles in terms of incidence correspondences - with infinite codimension - in certain Banach flag and Grassman manifolds, by analogy with standard finite-dimensional constructions of algebraic geometry [27]. Section 4.1 describes general incidence correspondences using Banach Grassman manifolds and $\S 4.2$ reinterprets these correspondences using spaces of Fredholm operators, which are more suitable for our purposes. In $\S 4.3$ we define the loci of irreducible, rank-one PU(2) monopoles in terms of these incidence correspondences. These rank-one loci are in turn detected with aid of the Dirac-operator period map, which is defined and whose differential is shown to have the requisite surjectivity properties in $\S 4.4$. Section 4.5 contains a proof of the parametric transversality theorem: the result is well-known, but we include the proof as no single reference contains the precise statement (and proof) we need. Finally, in $\S 4.6$, we complete the proof that the moduli spaces of irreducible $\mathrm{PU}(2)$ monopoles contain no rank-one pairs for generic parameters: we apply the parametric transversality theorem to show, in essence, that the loci of irreducible, rank-one PU(2) monopoles must have infinite codimension in their respective moduli spaces 
for generic parameters and so these loci are empty. The stratification of the space of Fredholm operators (by kernel dimension) [34], employed in $\S 4.6$, was also used by Maier in [45]. At the beginning of $\S 4.6$ we first give an outline of the main argument completing the proof of Theorem 4.1, as the detailed argument is rather long and technical.

Theorem 4.1 serves the auxiliary purpose of giving another method of completing the gap in the transversality argument used by PidstrigachTyurin in their definition of the $\operatorname{spin}^{c}$ polynomial invariants $[58, \S I]$; the same end is achieved via the holonomy perturbations of [16]. We briefly describe this application and Teleman's counterexample in the Appendix, together with some facts we need from linear algebra.

\subsection{Other approaches to transversality.}

As we remarked at the beginning of the Introduction, versions of Theorem 1.1 and 1.3 were proved by the author and Leness in [16] for the PU(2) monopole equations (1.1) with additional holonomy perturbations; see [18] for a more concise account of this method. The possible presence or absence of irreducible, rank-one solutions to the PU(2) monopole equations considered in [16] makes no difference to the argument there, as the perturbations are strong enough to yield transversality without a separate analysis of the locus of irreducible, rank-one solutions. A preliminary version [17] of the article [16] relied on the incorrect assertion of [58, p. 277] described above and used only the perturbation parameters $(\tau, \vartheta)$. Transversality results for the $\mathrm{PU}(2)$ monopole equations, with perturbation parameters including $(\tau, \vartheta)$ and the Riemannian metric $g$ on $T^{*} X$, were conjectured by Pidstrigach and Tyurin in [56], [59].

Teleman has explored another approach to the transversality problem, quite different from those of [16], [59], using certain ad hoc perturbations of the principal symbol of the linearization of the $\mathrm{PU}(2)$ monopole equations [71]. Like the PU(2) monopole equations (1.1), the equations of [71] employ the perturbation parameters $(\tau, \vartheta)$, together with a term of the form $\sum_{i} \rho^{-1} \tau_{i} \nabla_{A, Y_{i}}\left(\Phi \otimes \Phi^{*}\right)_{00}$ in the curvature equation in (1.1), where $\left\{Y_{i}\right\}$ is a finite set of vector fields spanning $T X$ at every point of $X$ and the coefficients $\tau_{i}$ are in $\Omega^{0}\left(\mathfrak{g l}\left(\mathfrak{s u}\left(W^{+}\right)\right)\right)$. The approach of [71] illustrates the significance of principal-symbol perturbations. However, as noted in the introduction to [72], it appears to be difficult or impossible to show that solutions to the $\mathrm{PU}(2)$ monopole equations of [71] which are reducible on an open subset of $X$ are necessarily reducible over all of $X$, as required by the transversality argument in [71]. Indeed, Proposition 3.1.2 in [71] assumes incorrectly 
that that the Agmon-Nirenberg unique continuation theorem for an ordinary differential equation on a Hilbert space [3] applies to the PU(2) monopole equations of [71], as an examination of the hypotheses of [3] reveals; the Agmon-Nirenberg theorem is used by Donaldson-Kronheimer in their proof of the corresponding unique continuation property for solutions to the antiself-dual equation and by the author and Leness for our proof of a restricted unique continuation property for the holonomy-perturbed $\mathrm{PU}(2)$ monopole equations of [16]. Varying the Riemannian metric $g$ on $T^{*} X$ and the Clifford map $\rho: T^{*} X \rightarrow \operatorname{Hom}_{\mathbb{C}}\left(W^{+}, W^{-}\right)$by automorphisms $f$ of $T^{*} X$, as we do in $\S 3$, perturbs the principal symbol of the Dirac operator; the key application of this perturbation occurs in the proof of Proposition 4.10, where we show that a certain partial differential of the Dirac-operator period map is surjective. Variations of the Dirac operator with respect to the Riemannian metric have been described by Bourguignon and Gauduchon in [7]. Their technique, with some enhancements, was recently used by Maier to prove certain generic metrics results for Dirac operators on low-dimensional spin or $\operatorname{spin}^{c}$ manifolds [45]. The variational formulas of [7], [45] were helpful for the development of our present article, though the variation of the Dirac operator we construct in $\S 3$ is quite different from that of [7]. The unique continuation property for reducible solutions to the anti-self-dual equation is an important ingredient in the proof of the Freed-Uhlenbeck generic metrics theorem given in [15] (see their Lemma 4.3.21); it is proved for the $\mathrm{PU}(2)$ monopole equations (1.1) by the author and Leness in [16] (see Theorem 4.11 in 4.4 here for a precise statement) and plays an important role in the proof of Theorem 1.3 here as well.

The present article was completed by August 1997, at which time it was widely circulated and submitted to a print journal. Shortly after this, the author received a preprint [72] from A. Teleman addressing, independently, the issue of transversality for the $\mathrm{PU}(2)$ monopole equations with perturbations similar to those we employ here.

\section{Acknowledgements.}

It is a pleasure to thank Thomas Leness for his generous support during the preparation of this article. We are very grateful to Tom Mrowka and Peter Ozsváth for their encouragement and many helpful comments. We warmly thank Clifford Taubes for his help and encouragement over the years; we also thank him and Dieter Kotschick for bringing the work of Stefan Maier concerning generic metrics results for Dirac operators to our attention and 
thank Stefan for sending us the preprint version of [45]. The preprint [71] of Andrei Teleman played a crucial role in the development of our article by pinpointing the gap in [58]. We would like to thank the Mathematics Departments at Harvard and Ohio State University and the National Science Foundation for their generous sponsorship during the preparation of this article.

\section{Transversality on the complement of the loci of lower-rank $\mathrm{PU}(2)$ monopoles.}

Let $\mathcal{C}_{W, E}^{*, \natural} \subset \mathcal{C}_{W, E}$ be the open subspace given by gauge-equivalence classes of pairs $(A, \Phi)$ with $A$ irreducible and $\Phi$ rank two, and set

$$
M_{W, E}^{*, \natural}:=M_{W, E} \cap \mathcal{C}_{W, E}^{*, \natural}
$$

Our goal in this section is to show that $M_{W, E}^{*, h}(\tau, \vartheta)$ is a regular manifold of the expected dimension for generic $C^{\infty}$ parameters $(\tau, \vartheta)$ : this result, Theorem 2.2, is proved in $\S 2.1$ under the assumption that the parametrized moduli space is regular while the latter result, Theorem 2.1, is proved in $\S 2.2$.

\subsection{The parametrized moduli space.}

Our argument relies, as with most standard applications in gauge theory [15], [23], [37], [74], on the Sard-Smale theorem for a Fredholm map of Banach manifolds [65].

We shall need to be precise here and throughout our article about the sense in which a parameter is 'generic'. A subset $S$ of a topological space $\mathcal{P}$ is called a set of the first category ${ }^{2}$ if its complement $\mathcal{P}-S$ is a countable intersection of dense open sets or, equivalently, if $S$ is a countable union of closed subsets of $\mathcal{P}$ with empty interior; if $\mathcal{P}$ is a complete metric space, then Baire's theorem implies that $\mathcal{P}-S$ is dense in $\mathcal{P}$ [62]. In our applications, $\mathcal{P}$ will either be a Banach or Fréchet manifold (with a complete metric), so $\mathcal{P}-S$ will always be ciense if $S$ is a first-category subset.

Throughout this section we use a Banach manifold of $C^{r}$ perturbation

\footnotetext{
${ }^{2} \mathrm{~A}$ second-category subset is not necessarily the complement of a first-category subset - although the term is sometimes inaccurately used that way: it is simply a subset which is not of the first category [62]. To eliminate any possible confusion, we avoid using the term here.
} 
parameters (with $r$ large) given by

$$
\mathcal{P}:=C^{r}\left(\mathrm{GL}\left(\Lambda^{+}\right)\right) \oplus C^{r}\left(\Lambda^{1} \otimes \mathbb{C}\right) .
$$

We could, of course, also include the space $C^{r}\left(\mathrm{GL}\left(T^{*} X\right)\right)$ of perturbations of the Riemannian metric on $X$ and of the Clifford map in our parameter space (2.1), as the latter perturbations are required for our period-map argument in $\S 4$. However, these additional parameters are not used to prove the main result of this section (Theorem 2.2), so we omit them for the sake of clarity. In the same vein, the space $C^{r}\left(\mathrm{GL}\left(\Lambda^{+}\right)\right)$of perturbation parameters $\tau$ is not required in our proof of the main result of $\S 4$ (Theorem 4.1), so in that section we omit the space $C^{r}\left(\mathrm{GL}\left(\Lambda^{+}\right)\right)$from the definition of the Banach manifold of perturbation parameters used there, again for reasons of clarity. To conclude the proof of our main transversality result (Theorem 1.3), though, we can clearly assume that the same space of perturbations (containing the three types of parameters) has been used throughout sections 2 and 4.

We define a ${ }^{\circ} \mathcal{G}_{E^{-}}$equivariant map

$$
\underline{\mathfrak{S}}:=\left(\underline{\mathfrak{S}}_{1}, \underline{\mathfrak{S}}_{2}\right): \mathcal{P} \times \tilde{\mathcal{C}}_{W, E} \rightarrow L_{k-1}^{2}\left(\Lambda^{+} \otimes(\mathfrak{s u}(E)) \oplus L_{k-1}^{2}\left(W^{-} \otimes E\right)\right.
$$

by setting

$$
\underline{\mathfrak{S}}(\tau, \vartheta, A, \Phi)=\left(\begin{array}{c}
\underline{\mathfrak{S}}_{1}(\tau, \vartheta, A, \Phi) \\
\underline{\mathfrak{S}}_{2}(\tau, \vartheta, A, \Phi)
\end{array}\right):=\left(\begin{array}{c}
F_{A}^{+}-\tau \rho^{-1}\left(\Phi \otimes \Phi^{*}\right)_{00} \\
\mathcal{D}_{A} \Phi+\rho(\vartheta) \Phi
\end{array}\right),
$$

where $(A, \Phi)$ is a pair on $\left(\mathfrak{s u}(E), W^{+} \otimes E\right)$ and the isomorphism ad $: \mathfrak{s u}(E) \simeq$ $\mathfrak{s o}(\mathfrak{s u}(E))$ is implicit, ${ }^{\circ} \mathcal{G}_{E}$ acts trivially on the space of perturbations $\mathcal{P}$, and so $\underline{\mathfrak{S}}^{-1}(0) /{ }^{\circ} \mathcal{G}_{E}$ is a subset of $\mathcal{P} \times \mathcal{C}_{W, E}$. We let $\mathfrak{M}_{W, E}$ denote the parametrized moduli space $\underline{\mathfrak{S}}^{-1}(0) /{ }^{\circ} \mathcal{G}_{E}$ and let $\mathfrak{M}_{W, E}^{*, \mathfrak{h}}:=\mathfrak{M}_{W, E} \cap\left(\mathcal{P} \times \mathcal{C}_{W, E}^{*, h}\right)$. We fix a $C^{\infty}$ Riemannian metric $g$ on $T^{*} X$, noting that it is not varied in this section.

The ${ }^{\circ} \mathcal{G}_{E}$-equivariant map $\underline{\mathfrak{S}}$ defines a section of a Banach vector bundle $\underline{\mathfrak{V}}$ over $\mathcal{P} \times \mathcal{C}_{W, E}^{*, \mathfrak{G}}$ with total space

$$
\underline{\mathfrak{V}}:=\mathcal{P} \times \tilde{\mathcal{C}}_{W, E}^{*, \mathfrak{\natural}} \times{ }_{\circ} \mathcal{G}_{E}\left(L_{k-1}^{2}\left(\Lambda^{+} \otimes \mathfrak{s u}(E)\right) \oplus L_{k-1}^{2}\left(W^{-} \otimes E\right)\right),
$$

so $\mathfrak{S}:=\underline{\mathfrak{S}}(\tau, \vartheta, \cdot)$ is a section over $\mathcal{C}_{W, E}^{*, h}$ of the Banach vector bundle $\mathfrak{V}:=$ $\left.\mathfrak{V}\right|_{(\tau, \vartheta)}$. In particular, the parametrized moduli space $\mathfrak{M}_{W, E}^{*, \mathfrak{G}}$ is the zero set of the section $\underline{\mathfrak{S}}$ of the vector bundle $\underline{\mathfrak{V}}$ over $\mathcal{P} \times \mathcal{C}_{W, E}^{*, \mathfrak{h}}$.

Theorem 2.1. The zero set in $\mathcal{P} \times \mathcal{C}_{W, E}^{*, \mathfrak{h}}$ of the section $\underline{\mathfrak{S}}$ is regular and, in particular, the moduli space $\mathfrak{M}_{W, E}^{*, \downarrow}$ is a smooth Banach submanifold of $\mathcal{P} \times \mathcal{C}_{W, E}^{*, \natural}$. 
To preserve continuity, we defer the proof of Theorem 2.1 to $\S 2.2$. The differential $D \underline{\mathfrak{S}}:=(D \underline{\mathfrak{S}})_{[\tau, \vartheta, A, \Phi]}$ of the section $\underline{\mathfrak{S}}$ at a point $[\tau, \vartheta, A, \Phi]$ in $\mathcal{P} \times \mathcal{C}_{W, E}^{*, h}$ is given by

$$
D \underline{\mathfrak{S}}(\delta \tau, \delta \vartheta, a, \phi)=\left(\begin{array}{l}
D \underline{\mathfrak{S}}_{1}(\delta \tau, \delta \vartheta, a, \phi) \\
D \underline{\mathfrak{S}}_{2}(\delta \tau, \delta \vartheta, a, \phi)
\end{array}\right)
$$

where $(a, \phi) \in \operatorname{Ker} d_{A, \Phi}^{0, *} \subset L_{k}^{2}\left(\Lambda^{1} \otimes \mathfrak{s u}(E)\right) \oplus L_{k}^{2}\left(W^{+} \otimes E\right)$ represents a vector in the tangent space $\left(T \mathcal{C}_{W, E}^{*, \natural}\right)_{[A, \Phi]}=T_{[A, \Phi]} \mathcal{C}_{W, E}^{*, \natural}$ and $(\delta \tau, \delta \vartheta) \in \mathcal{P}$. Here, $d_{A, \Phi}^{0, *}$ is the $L^{2}$ adjoint of the differential

$$
d_{A, \Phi}^{0}: L_{k+1}^{2}(\mathfrak{s u}(E)) \oplus i \mathbb{R}_{Z} \rightarrow L_{k}^{2}(\mathfrak{s u}(E)) \oplus L_{k}^{2}\left(W^{+} \otimes E\right)
$$

of the map ${ }^{\circ} \mathcal{G}_{E} \rightarrow \tilde{\mathcal{C}}_{W, E}, u \mapsto u(A, \Phi)$, at the identity.

The differentials in (2.3) are given explicitly by

$$
\begin{aligned}
D \underline{\mathfrak{S}}_{1}(\delta \tau, \delta \vartheta, a, \phi)= & d_{A}^{+} a-(\delta \tau) \tau \rho^{-1}\left(\Phi \otimes \Phi^{*}\right)_{00} \\
& -\tau \rho^{-1}\left(\phi \otimes \Phi^{*}+\Phi \otimes \phi^{*}\right)_{00} \\
D \underline{\mathfrak{S}}_{2}(\delta \tau, \delta \vartheta, a, \phi)= & \mathcal{D}_{A} \phi+\rho(\vartheta) \phi+\rho(a) \Phi+\rho(\delta \vartheta) \Phi .
\end{aligned}
$$

We have $D \underline{\mathfrak{S}}\left(\cdot, d_{A, \Phi}^{0} \zeta\right)=0$ for all $\zeta \in L_{k+1}^{2}(\mathfrak{s u}(E)) \oplus i \mathbb{R}_{Z}$ since $\underline{\mathfrak{S}}$ is ${ }^{\circ} \mathcal{G}_{E^{-}}$ equivariant. By the regularity results of $[16, \S 3]$ we may assume, without loss of generality, that the pair $(A, \Phi)$ in $\tilde{\mathcal{C}}_{W, E}^{*, \mathrm{~h}}$ is a $C^{r}$ representative for the point $[A, \Phi]$ in the zero set $\mathfrak{S}^{-1}(0) \subset \mathcal{C}_{W, E}^{*, \natural}$. Since the tangent space $\left(T \mathcal{C}_{W, E}^{*, \natural}\right)_{[A, \Phi]}$ may be identified with $\operatorname{Ker} d_{A, \Phi}^{0, *}$ by the slice result [16, Proposition 2.8], we have

$$
D \underline{\mathfrak{S}}(0,0, a, \phi):=d_{A, \Phi}^{1}(a, \phi)=\left(d_{A, \Phi}^{0, *}+d_{A, \Phi}^{1}\right)(a, \phi),
$$

for $(a, \phi) \in \operatorname{Ker} d_{A, \Phi}^{0, *}$, so the differential $\left.D \underline{\mathfrak{S}}\right|_{\{0\} \times T \mathcal{C}_{W, E}^{*, \natural}}$ is Fredholm, where $\{0\} \times T \mathcal{C}_{W, E}^{0, *}=T\left(\{\tau, \vartheta\} \times \mathcal{C}_{W, E}^{*, h}\right)$. We recall that $d_{A, \Phi}^{0}$ and $d_{A, \Phi}^{1}$ are the two differentials in the elliptic deformation complex for the PU 2) monopole equations (1.1). Thus, $\underline{\mathfrak{S}}$ is a Fredholm section when restricted to the fixedparameter fibers $\{\tau, \vartheta\} \times \mathcal{C}_{W, E}^{*, \downarrow} \subset \mathcal{P} \times \mathcal{C}_{W, E}^{*, \natural}$ and so the Sard-Smale theorem (in the form of Proposition 4.3.11 in [15]) implies that there is a first-category subset $\mathcal{P}_{\mathrm{fc}} \subset \mathcal{P}$ such that the zero sets in $\mathcal{C}_{W, E}^{*, \natural}$ of the sections $\mathfrak{S}:=\mathfrak{S}(\tau, \vartheta, \cdot)$ are regular for all perturbations $(\tau, \vartheta) \in \mathcal{P}-\mathcal{P}_{\mathrm{fc}}$. Now

$$
M_{W, E}^{*, \natural}(\tau, \vartheta)=\mathfrak{S}^{-1}(0) \cap \mathcal{C}_{W, E}^{*, \natural}
$$


and so for generic $C^{r}$ parameters $(\tau, \vartheta)$, the moduli space $M_{W, E}^{*, h}(\tau, \vartheta)$ is a smooth manifold. Finally, the argument of $[16, \S 5.1 .2]$ implies that the parameters $(g, \tau, \vartheta)$ can be assumed without loss of generality to be $C^{\infty}$ if $X$ is a smooth manifold. Let

$$
\mathcal{P}^{\infty}:=C^{\infty}\left(\mathrm{GL}\left(\Lambda^{+}\right)\right) \times C^{\infty}\left(\Lambda^{1} \otimes \mathbb{C}\right),
$$

denote the Fréchet manifold of $C^{\infty}$ perturbation parameters. The expected dimension of $M_{W, E}^{*, \downarrow}(\tau, \vartheta)$ is given by Proposition 2.28 in [16] and is obtained by computing the index of the elliptic deformation complex for the $\mathrm{PU}(2)$ monopole equations (1.1). In summary, we have:

Theorem 2.2. Let $X$ be a closed, oriented, smooth four-manifold with $C^{\infty}$ Riemannian metric. Then there is a first-category subset $\mathcal{P}_{\mathrm{fc}}^{\infty} \subset \mathcal{P}^{\infty}$ such that for all $(\tau, \vartheta)$ in $\mathcal{P}^{\infty}-\mathcal{P}_{\mathrm{fc}}^{\infty}$ the following holds: The zero set of the section $\mathfrak{S}:=\underline{\mathfrak{S}}(\tau, \vartheta, \cdot)$ is regular and the moduli space $M_{W, E}^{*, 4}(\tau, \vartheta)=\mathfrak{S}^{-1}(0) \cap \mathcal{C}_{W, E}^{*, 4}$ is a smooth submanifold of of the expected dimension.

\subsection{Smoothness of the parametrized moduli space.}

We prove Theorem 2.1 in this section by showing that the ${ }^{\circ} \mathcal{G}_{E}$-equivariant $\operatorname{map} \underline{\mathfrak{S}}: \mathcal{P} \times \tilde{\mathcal{C}}_{W, E}^{*, \underline{h}} \rightarrow L_{k-1}^{2}\left(\Lambda^{+} \otimes \mathfrak{s u}(E)\right) \oplus L_{k-1}^{2}\left(W^{-} \otimes E\right)$ vanishes transversely and so the parametrized moduli space $\mathfrak{M}_{W, E}^{*, h}=\underline{\mathfrak{S}}^{-1}(0) /{ }^{\circ} \mathcal{G}_{E}$ is a smooth Banach manifold. We may suppose without loss of generality that $(\tau, \vartheta, A, \Phi)$ is a $C^{r}$ representative for a point in $\underline{\mathfrak{S}}^{-1}(0)$ and denote $D \underline{\mathfrak{S}}:=(D \underline{\mathfrak{S}})_{\tau, \vartheta, A, \Phi}$ for convenience.

Note that $\operatorname{Ran} D \underline{\mathfrak{S}}_{2}$ is a real subspace of $L_{k-1}^{2}\left(W^{-} \otimes E\right)$ according to (2.5): indeed, $D \underline{\mathfrak{S}}_{2}(\delta \tau, 0, a, 0)$ spans a real subspace as $a$ varies, while $D \underline{\mathfrak{S}}_{2}(\delta \tau, \delta \vartheta, 0, \phi)$ spans a complex subspace as $\delta \vartheta \phi$. Hence,

$$
\operatorname{Ran}(D \underline{\mathfrak{s}}) \varsubsetneqq L_{k-1}^{2}\left(\Lambda^{+} \otimes \mathfrak{s u}(E)\right) \oplus L_{k-1}^{2}\left(W^{-} \otimes E\right)
$$

if and only if there exists a non-zero pair $(v, \psi)$ such that for all $(\delta \tau, \delta \vartheta, a, \phi)$ we have

$$
\begin{aligned}
& (D \underline{\mathfrak{S}}(\delta \tau, \delta \vartheta, a, \phi),(v, \psi))_{L^{2}(X)} \\
& =\left(D \underline{\mathfrak{S}}_{1}(\delta \tau, \delta \vartheta, a, \phi), v\right)_{L^{2}(X)} \\
& +\operatorname{Re}\left(D \underline{\mathfrak{S}}_{2}(\delta \tau, \delta \vartheta, a, \phi), \psi\right)_{L^{2}(X)}=0 .
\end{aligned}
$$

Suppose that $(v, \psi) \in L_{k-1}^{2}\left(\Lambda^{+} \otimes \mathfrak{s u}(E)\right) \oplus L_{k-1}^{2}\left(W^{-} \otimes E\right)$ is real $L^{2}$ orthogonal to $\operatorname{Ran} D \underline{\mathfrak{S}}$, so $(v, \psi)$ lies in $\operatorname{Ker}(D \underline{\mathfrak{S}})^{*}$ by $(2.6)$. Then elliptic 
regularity for the Laplacian $D \underline{\mathfrak{S}}(D \underline{\mathfrak{S}})^{*}$, with $C^{r-1}$ coefficients, implies that $(v, \psi)$ is in $C^{r+1}[16, \S 3]$. Moreover, Aronszajn's theorem [5, Remark 3, p. 248], [31, Theorem 1.8] implies that pairs $(v, \psi)$ in the kernel of $D \underline{\mathfrak{S}}(D \underline{\mathfrak{S}})^{*}$ have the unique continuation property [16, Lemma 5.9].

We begin with a simple observation from linear algebra; though elementary, it has an important application in the Freed-Uhlenbeck proof of the generic metrics theorem for the anti-self-dual equation (see, for example, Lemma 3.7 in [23]). We shall make extensive use of it here.

Lemma 2.3. Let $U, V, W$ be finite-dimensional Hilbert spaces (either all real or all complex) with $\operatorname{dim} U \leq \operatorname{dim} V$. Suppose $M \in \operatorname{Hom}(U, W)=$ $W \otimes U^{*}$ and $N \in \operatorname{Hom}(V, W)=W \otimes V^{*}$. If $\langle M P, N\rangle_{W \otimes V^{*}}=0$ for all $P \in \operatorname{Hom}(V, U)$, then $\operatorname{Ran} M \perp \operatorname{Ran} N$ in $W$ and so $\operatorname{Rank} M+\operatorname{Rank} N \leq$ $\operatorname{dim} W$.

Proof. Let $\left\{v_{i}\right\}$ be an orthonormal basis for $V$, with dual orthonormal basis $\left\{v_{i}^{*}\right\}$ for $V^{*}$ given by $v_{i}^{*}:=\left\langle\cdot, v_{i}\right\rangle$, and let $\left\{u_{j}\right\}$ be an orthonormal basis for $U$. We then have

$$
\begin{aligned}
0 & =\langle M P, N\rangle_{W \otimes V^{*}} \\
& =\sum_{i, j}\left\langle M P v_{i} \otimes v_{i}^{*}, N v_{j} \otimes v_{j}^{*}\right\rangle_{W \otimes V^{*}} \\
& =\sum_{i, j}\left\langle M P v_{i}, N v_{j}\right\rangle_{W} \cdot\left\langle v_{i}^{*}, v_{j}^{*}\right\rangle_{V^{*}} \\
& =\sum_{i}\left\langle M P v_{i}, N v_{i}\right\rangle_{W} .
\end{aligned}
$$

Since $P \in \operatorname{Hom}(V, U)$ is arbitrary, for each $i^{\prime} \in\{1, \ldots, \operatorname{dim} V\}$ and $j \in$ $\{1, \ldots, \operatorname{dim} U\}$ we may choose $P=P_{i^{\prime} j}$ such that $P_{i^{\prime} j} v_{i}=0$ for all $i \neq i^{\prime}$ and $P_{i^{\prime} j} v_{i^{\prime}}=u_{j}$, so the preceding identity implies that

$$
\left\langle M u_{j}, N v_{i^{\prime}}\right\rangle_{W}=0, \quad \text { for all } 1 \leq i^{\prime} \leq \operatorname{dim} V \text { and } 1 \leq j \leq \operatorname{dim} U .
$$

Hence, $\langle M u, N v\rangle_{W}=0$ for all $u \in U, v \in V$, and the assertion follows.

Setting $(\delta \tau, a, \phi)=0$ in (2.4), (2.5), and (2.6) yields

$$
\operatorname{Re}(\rho(\delta \vartheta) \Phi, \psi)_{L^{2}(X)}=0, \quad \text { for all } \delta \vartheta \in C^{r}\left(\Lambda^{1} \otimes \mathbb{C}\right) .
$$

Using the identity $z=\operatorname{Re}(z)+i \operatorname{Re}(-i z)$ for $z \in \mathbb{C}$, we see that

$$
(\rho(\delta \vartheta) \Phi, \psi)_{L^{2}(X)}=\operatorname{Re}(\rho(\delta \vartheta) \Phi, \psi)_{L^{2}(X)}+i \operatorname{Re}(\rho(-i \delta \vartheta) \Phi, \psi)_{L^{2}(X)}
$$


and so we obtain the full complex $L^{2}$-orthogonality condition

$$
(\rho(\delta \vartheta) \Phi, \psi)_{L^{2}(X)}=0, \quad \text { for all } \delta \vartheta \in C^{r}\left(\Lambda^{1} \otimes \mathbb{C}\right) .
$$

Recall that the ranks of sections of the complex bundle $W^{+} \otimes E$ and real bundles $\mathfrak{s u}\left(W^{+}\right) \otimes \mathfrak{s u}(E)$ and $\Lambda^{+} \otimes \mathfrak{s u}(E)$ are defined by considering them as sections of $\operatorname{Hom}_{\mathbb{C}}\left(W^{+, *}, E\right), \operatorname{Hom}_{\mathbb{R}}\left(\mathfrak{s u}\left(W^{+}\right)^{*}, \mathfrak{s u}(E)\right)$, and $\operatorname{Hom}_{\mathbb{R}}\left(\Lambda^{+, *}, \mathfrak{s u}(E)\right)$, respectively.

Lemma 2.4 ([17]). Suppose $\Phi \in C^{0}\left(X, W^{+} \otimes E\right)$ and $\Psi \in C^{0}\left(X, W^{-} \otimes E\right)$ satisfy (2.7). Then $\Phi$ and $\Psi$, considered as elements of $\operatorname{Hom}_{\mathbb{C}}\left(W^{+, *}, E\right)$ and $\operatorname{Hom}_{\mathbb{C}}\left(W^{-, *}, E\right)$ respectively, have complex-orthogonal images in $E$ at every point of $X$ and thus $\operatorname{Rank}_{\mathbb{C}} \Phi(x)+\operatorname{Rank}_{\mathbb{C}} \Psi(x) \leq 2$ at each point $x \in X$.

Proof. Since $\vartheta$ is an arbitrary, $C^{r}$ complex-valued one-form, we obtain the pointwise identity $\left\langle\rho\left(\vartheta_{x}\right) \Phi_{x}, \Psi_{x}\right\rangle_{x}=0$ for all $\vartheta_{x} \in\left(T^{*} X\right)_{x} \otimes \mathbb{C}$. Let $\left\{\phi_{1}, \phi_{2}\right\}$, $\left\{\psi_{1}, \psi_{2}\right\}$ be orthonormal frames for $\left.W^{+}\right|_{x},\left.W^{-}\right|_{x}$, respectively. The Clifford map is complex linear and restricts to give a complex-linear isomorphism $[48$, p. 89],

$$
\rho:\left(T^{*} X\right)_{x} \otimes_{\mathbb{R}} \mathbb{C} \rightarrow \operatorname{Hom}_{\mathbb{C}}\left(W^{+}, W^{-}\right)_{x} .
$$

(This is proved as Lemma A.4 in Appendix A.3.) The conclusion now follows from the first assertion in Lemma 2.3.

Since $(A, \Phi) \in \tilde{\mathcal{C}}_{W, E}^{*, \natural}$ by hypothesis and $\Phi$ is $C^{r}$, there is a non-empty open subset $U \subset X$ on which $\operatorname{Rank}_{\mathbb{C}} \Phi(x)=2$, for all $x \in U$. Equation (2.7) and Lemma 2.4 imply that $\operatorname{Rank}_{\mathbb{C}} \psi(x)=0$ for all $x \in U$, that is, $\psi \equiv 0$ on $U$. Similarly, by varying $\tau$ we see that (2.4), (2.5), and (2.6) yield

$$
\left((\delta \tau) \tau \rho^{-1}\left(\Phi \otimes \Phi^{*}\right)_{00}, v\right)_{L^{2}(X)}=0, \quad \text { for all } \delta \tau \in C^{r}\left(\mathfrak{g l}\left(\Lambda^{+}\right)\right),
$$

by setting $(\delta \vartheta, a, \phi)=0$.

Remark 2.5. Even though $\Phi$ solves an elliptic equation, namely $\left(\mathcal{D}_{A}+\right.$ $\rho(\vartheta)) \Phi=0$, and $\operatorname{Rank}_{\mathbb{C}} \Phi(x)=2$ for all $x$ in the open set $U$, it does not necessarily follow that $\operatorname{Rank}_{\mathbb{C}} \Phi(x)=2$ at all points $x$ in $X$; see [29] and [31, p. 668] for counterexamples in the case of harmonic maps. If all the perturbation parameters are analytic and $(X, g)$ is a real-analytic Riemannian manifold then $\Phi$ will necessarily be real-analytic by [50, Theorem 6.6.1], so $\operatorname{det} \Phi$ (see $\S 4$ ) will be a complex-valued, real-analytic function and if it vanishes on a non-empty open set, it will vanish identically. However, we cannot 
constrain our parameters to be real-analytic before applying the Sard-Smale theorem (as a space of real-analytic parameters would not have a complete metric), so we make no use of real-analyticity here.

Lemma 2.6 ([17]). If $v \in C^{0}\left(\Lambda^{+} \otimes \mathfrak{s u}(E)\right)$ and $\Phi \in C^{0}\left(W^{+} \otimes E\right)$ satisfy (2.8), then $v, \tau \rho^{-1}\left(\Phi \otimes \Phi^{*}\right)_{00} \in \operatorname{Hom}_{\mathbb{R}}\left(\Lambda^{+, *}, \mathfrak{s u}(E)\right)$ have orthogonal images in $\mathfrak{s u}(E)$ at every point in $X$ and so $\operatorname{Rank}_{\mathbb{R}} v(x)+\operatorname{Rank}_{\mathbb{R}}\left(\Phi(x) \otimes \Phi(x)^{*}\right)_{00} \leq 3$ at each $x \in X$.

Proof. Since $\delta \tau \in C^{r}\left(\mathfrak{g l}\left(\Lambda^{+}\right)\right)$is arbitrary, we obtain the pointwise identity

$$
\left\langle\left(\delta \tau_{x}\right) \tau \rho^{-1}\left(\Phi \otimes \Phi^{*}\right)_{00}, v\right\rangle_{x}=0 \quad \text { for all } \delta \tau_{x} \in \mathfrak{g l}\left(\left.\Lambda^{+}\right|_{x}\right),
$$

and any $x \in X$. The conclusion now follows from the second assertion in Lemma 2.3.

Lemma 2.7 ([16, Lemma 2.21]). If $\Phi \in C^{0}\left(W^{+} \otimes E\right)$ and $x \in X$, then the following hold:

1. $\operatorname{Rank}_{\mathbb{R}}\left(\Phi(x) \otimes \Phi^{*}(x)\right)_{00}=1$ if and only if $\operatorname{Rank}_{\mathbb{C}} \Phi(x)=1$,

2. $\operatorname{Rank}_{\mathbb{R}}\left(\Phi(x) \otimes \Phi^{*}(x)\right)_{00}=3$ if and only if $\operatorname{Rank}_{\mathbb{C}} \Phi(x)=2$.

Remark 2.8. Note that if $\Phi$ is rank two on $X$, then $\left(\Phi \otimes \Phi^{*}\right)_{00}$ is rank three on $X$ and so (1.1) implies that $F_{A}^{+}$is rank three on $X$ when $(A, \Phi)$ is a PU(2) monopole: thus, $A$ cannot be a reducible connection on $X$.

We can now complete the proof of Theorem 2.1 in either of two ways; the interest in the second method is explained in the remark at the end of the proof.

First proof of Theorem 2.1. Since $\operatorname{Rank}_{\mathbb{C}} \Phi(x)=2$, for all $x \in U$, Lemma 2.7 implies that $\operatorname{Rank}_{\mathbb{R}}\left(\Phi(x) \otimes \Phi^{*}(x)\right)_{00}=3$, for all $x \in U$, and so Lemma 2.6 implies that $\operatorname{Rank}_{\mathbb{R}} v(x)=0$, for all $x \in U$, since $\mathfrak{s u}(E)$ is rank three and thus $v \equiv 0$ on $U$. Hence, $(v, \psi) \equiv 0$ on $U$ and so $(v, \psi) \equiv 0$ on $X$ by unique continuation for the Laplacian $D \underline{\mathfrak{s}} D \underline{\mathfrak{S}}^{*}$, as desired.

Alternatively, we observe that it is enough to know that $\left(\Phi \otimes \Phi^{*}\right)_{00}$ has at least rank two at some point of $X$ :

Second proof of Theorem 2.1. Since $\operatorname{Rank}_{\mathbb{C}} \Phi(x)=2$, for all $x \in U$, Lemma 2.7 implies that $\operatorname{Rank}_{\mathbb{R}}\left(\Phi(x) \otimes \Phi^{*}(x)\right)_{00} \geq 2$, for all $x \in U$, and so Lemma 
2.6 implies that $\operatorname{Rank}_{\mathbb{R}} v(x) \leq 1$, for all $x \in U$, since $\mathfrak{s u}(E)$ is rank three. On an open subset $U^{\prime} \subset X$ where $v \neq 0$, we can write $v=\varpi \otimes b$, where $\varpi \in C^{r}\left(U^{\prime}, \Lambda^{+}\right)$and $b \in C^{r}\left(U^{\prime}, \mathfrak{s u}(E)\right)$, with $|b|=1$ on $U^{\prime}$. The argument of $\left[15\right.$, Lemma 4.3.25] and [23, Proposition 3.4, p. 56] now implies that $d_{A} b=0$ on $U^{\prime}$. The connection $A$ is thus reducible on the non-empty open subset $U^{\prime} \subset X$, which we may assume to be connected without loss of generality. Theorem 4.11 implies that $A$, and so the pair $(A, \Phi)$, is reducible on all of $X$ - again contradicting our assumption that $(A, \Phi)$ is irreducible. Hence, $v \equiv 0$ on $X$ and so $(v, \psi) \equiv 0$ on $X$.

Remark 2.9. In conjunction with the variations of the Dirac operator considered in $\S 4$, a direct approach (avoiding the period map of $\S 4$ ) to the proof of our main transversality theorem would work were it not for the fact that it seems impossible (using these variations) to show that $v$ has at most rank one on some non-empty open subset of $X$, as used in the second proof above.

\section{Variation of the Dirac operator.}

The second main step in the proof of Theorem 1.3 is to show, for generic parameters, that if $(A, \Phi)$ is a $\mathrm{PU}(2)$ monopole then the element $\Phi$ of the kernel of the perturbed Dirac operator defined by $A$ cannot be rank one. In $\S 3.1$ we introduce our full parameter space of perturbations of the Dirac operator and compute the differential of the resulting family, while in $\S 3.2$ we describe the corresponding perturbed $\mathrm{PU}(2)$ monopole equations and the universal moduli space.

\subsection{Clifford maps and Dirac-operator variations.}

In order to describe our variations, it is helpful to have a construction of the Dirac operator at our disposal which is as simple as possible. The minimal, axiomatic approach employed by Kronheimer-Mrowka [39] and Mrowka-Ozsváth-Yu [51] is extremely useful for this purpose, so this is the approach we shall follow here.

Recall that a real-linear map $\rho_{+}: T^{*} X \rightarrow \operatorname{Hom}_{\mathbb{C}}\left(W^{+}, W^{-}\right)$defines a Clifford-algebra representation $\rho: \mathrm{Cl}_{\mathbb{C}}\left(T^{*} X\right) \rightarrow \operatorname{End}_{\mathbb{C}}(W)$, with $W:=$ $W^{+} \oplus W^{-}$, if and only if [42], [63]

$$
\rho_{+}(\alpha)^{\dagger} \rho_{+}(\alpha)=g(\alpha, \alpha) \operatorname{id}_{W^{+}}, \quad \alpha \in C^{\infty}\left(T^{*} X\right),
$$

where $g$ denotes the Riemannian metric on $T^{*} X$. The real-linear map $\rho$ : 
$T^{*} X \rightarrow \operatorname{End}_{\mathbb{C}}\left(W^{+} \oplus W^{-}\right)$is obtained by defining a real-linear map

$$
\rho_{-}: T^{*} X \rightarrow \operatorname{Hom}_{\mathbb{C}}\left(W^{-}, W^{+}\right), \quad \alpha \mapsto \rho_{-}(\alpha):=-\rho_{+}(\alpha)^{\dagger},
$$

and setting

$$
\rho(\alpha):=\left(\begin{array}{cc}
0 & \rho_{-}(\alpha) \\
\rho_{+}(\alpha) & 0
\end{array}\right) .
$$

The Clifford algebra representation $\rho: \operatorname{Cl}_{\mathbb{C}}\left(T^{*} X\right) \rightarrow \operatorname{End}_{\mathbb{C}}\left(W^{+} \oplus W^{-}\right)$is uniquely determined by the map $\rho_{+}: T^{*} X \rightarrow \operatorname{Hom}_{\mathbb{C}}\left(W^{+}, W^{-}\right)$and satisfies

$$
\rho(\alpha)^{\dagger}=-\rho(\alpha) \quad \text { and } \quad \rho(\alpha)^{\dagger} \rho(\alpha)=g(\alpha, \alpha) \operatorname{id}_{W}, \quad \alpha \in C^{\infty}\left(T^{*} X\right) .
$$

We may vary $\rho_{+}$by automorphisms of $T^{*} X$. If $f \in C^{\infty}\left(\mathrm{GL}\left(T^{*} X\right)\right)$, then

$$
\begin{aligned}
\rho_{+}(f(\alpha))^{\dagger} \rho_{+}(f(\alpha)) & =g(f(\alpha), f(\alpha)) \operatorname{id}_{W^{+}} \\
& =\left(f^{*} g\right)(\alpha, \alpha) \operatorname{id}_{W^{+}},
\end{aligned}
$$

and we obtain a Clifford map $\rho_{f,+}:=\rho_{+} \circ f: T^{*} X \rightarrow \operatorname{Hom}_{\mathbb{C}}\left(W^{+}, W^{-}\right)$ which is compatible with the Riemannian metric $f^{*} g$ on $T^{*} X$. Thus, for $f \in C^{\infty}\left(\mathrm{O}\left(T^{*} X\right)\right)$, the Clifford map $\rho_{f,+}$ is compatible with the given Riemannian metric $g$.

Recall from [48, p. 89] (or Lemma A.4), that the complexification of $\rho_{+}$ yields an isomorphism

$$
\rho_{+}:\left(T^{*} X\right) \otimes_{\mathbb{R}} \mathbb{C} \rightarrow \operatorname{Hom}_{\mathbb{C}}\left(W^{+}, W^{-}\right) .
$$

The space $C^{r}\left(\mathrm{GL}\left(T^{*} X\right)\right)$ is a Banach Lie group with Lie algebra $C^{r}\left(\mathfrak{g l}\left(T^{*} X\right)\right)$ [23], [55]. Then, for all $e \in \Omega^{1}(X, \mathbb{R})$ and $\Phi \in \Omega^{0}\left(W^{+}\right)$, we have

$$
\left(\left(D \rho_{f,+}\right)(\delta f)\right)(e) \Phi=\rho_{+}((\delta f) e) \Phi
$$

where $\delta f \in C^{r}\left(\mathfrak{g l}\left(T^{*} X\right)\right)$.

For the Riemannian metric $g$ on $T^{*} X$, let $\nabla^{g}$ be an $\mathrm{SO}(4)$ connection on $T^{*} X$. A unitary connection $\nabla$ on $W$ is called spinorial with respect to $\nabla^{g}$ if it induces the $\mathrm{SO}(4)$ connection $\nabla^{g}$ on $T^{*} X$, or, equivalently, if the covariant derivative $\nabla$ on $C^{\infty}(W)$ is a derivation with respect to Clifford multiplication, $\rho: C^{\infty}\left(\Lambda^{\bullet}\left(T^{*} X\right) \otimes W\right) \rightarrow C^{\infty}(W)$, so [42], [63]

$$
\nabla_{\eta}(\rho(\beta) \Phi)=\rho\left(\nabla_{\eta}^{g} \beta\right) \Phi+\rho(\beta) \nabla_{\eta} \Phi,
$$


for all $\eta \in C^{\infty}(T X), \beta \in \Omega^{\bullet}(X, \mathbb{R})$, and $\Phi \in C^{\infty}(W)$. (Our convention differs from that of [51, Definition 4.0.23].) The unitary connection $\nabla$ on $W$ uniquely determines a unitary connection on $\operatorname{det} W^{+} \simeq \operatorname{det} W^{-}$in the standard way [33]. Any two unitary connections on $W$, which are both spinorial with respect to $\nabla^{g}$, differ by an element of $\Omega^{1}(X, i \mathbb{R})$. Conversely, a unitary connection $\nabla$ on a Hermitian two-plane bundle $W=W^{+} \oplus W^{-}$ over an oriented four-manifold $X$ is uniquely determined by

- A Clifford map $\rho_{+}: T^{*} X \rightarrow \operatorname{Hom}_{\mathbb{C}}\left(W^{+}, W^{-}\right)$satisfying (3.1) for the Riemannian metric $g$ on $T^{*} X$,

- An $\mathrm{SO}(4)$ connection $\nabla^{g}$ on $T^{*} X$ for the metric $g$, which need not be torsion free, and,

- A U(1) connection $B$ on $\operatorname{det} W^{+}$.

The resulting connection $\nabla$ on $W$ is then spinorial with respect to $\nabla^{g}$.

Digressing slightly, we recall that the local connection matrix one-form of $\nabla$ may be expressed in terms of those of the connections on $T^{*} X$ and $\operatorname{det} W^{+}$. To see this, let $\left\{e^{i}\right\}$ be an oriented, $g$-orthonormal local frame for $T^{*} X$ with dual frame $\left\{e_{i}\right\}$ for $T X$ and let ${ }^{j} \omega_{k l}, j=1,2$, be the corresponding $\mathfrak{s o}$ (4) connection matrices for the $\mathrm{SO}(4)$ connections ${ }^{j} \nabla^{g}$ over an open subset $U \subset X$. Let $B \in \Omega^{1}(U, i \mathbb{R})$ also denote the local connection one-form for the $\mathrm{U}(1)$ connection on $\operatorname{det} W^{+}$, with respect to a trivialization for $\operatorname{det} W^{+}$ induced from that of $W^{+}$over $U$. From [28, p. 4] (see also [42, Theorem II.4.14]), the local connection matrix one-forms for the $\operatorname{spin}^{c}$ connections ${ }^{j} \nabla$ on $W$ defined by $\left(\rho,{ }^{j} \nabla^{g}, B\right)$ are given by

$$
\begin{aligned}
{ }^{j} \omega^{\operatorname{Spin}^{c}(4)} & ={ }^{j} \omega^{\operatorname{Spin}(4)}+\frac{1}{2} B \operatorname{id}_{W^{+}} \\
& =\frac{1}{4} \sum_{k, l}{ }^{j} \omega_{k l} \otimes \rho\left(e^{k} \wedge e^{l}\right)+\frac{1}{2} B \operatorname{id}_{W^{+}} .
\end{aligned}
$$

Although the formula of [28] refers only to spin connections, locally we may write $\left.W\right|_{U}=S \otimes_{\mathbb{C}} N$, where $S$ is a spin bundle for the local spin structure and $N$ is a Hermitian line bundle such that $N^{\otimes 2}=\left.\operatorname{det} W^{+}\right|_{U}$. We may then write the $\operatorname{spin}^{c}$ connections on $\left.W\right|_{U}$ as tensor products of spin connections on $S$ with connection matrix one-forms ${ }^{j} \omega^{\mathrm{Spin}(4)}$ and a $\mathrm{U}(1)$ connection on $N$ with connection one-form $\frac{1}{2} B$, as above.

Given a unitary connection $A$ on an auxiliary Hermitian two-plane bundle $E$, we let $\nabla_{A}$ denote the induced unitary connection on $W \otimes E$. The corresponding Dirac operator $[26, \S 3.4]$,

$$
\mathcal{D}_{A}:=\rho_{+} \circ \nabla_{A},
$$


is defined by the composition of the covariant derivative, $\nabla_{A}: C^{\infty}\left(W^{+} \otimes\right.$ $E) \rightarrow C^{\infty}\left(T^{*} X \otimes W^{+} \otimes E\right)$, and Clifford multiplication, $\rho_{+}: C^{\infty}\left(T^{*} X \otimes\right.$ $\left.W^{+} \otimes E\right) \rightarrow C^{\infty}\left(W^{-} \otimes E\right.$ ). If $\left\{v^{i}\right\}$ is a (not necessarily $g$-orthonormal) local frame for $T^{*} X$ with dual frame $\left\{v_{i}\right\}$ for $T X$, defined by $v^{i}\left(v_{j}\right)=\delta_{i j}$, then $\nabla_{A}=\sum_{i=1}^{4} v^{i} \otimes \nabla_{A, v_{i}}$ and so $\mathcal{D}_{A}$ has the familiar shape

$$
\mathcal{D}_{A}=\sum_{i=1}^{4} \rho_{+}\left(v^{i}\right) \nabla_{A, v_{i}} .
$$

It is very convenient to keep the unitary connection $\nabla$ on $W$ fixed throughout, so while $(\nabla, \rho)$ necessarily induces an $\mathrm{SO}(4)$ connection $\nabla^{g}$ on $T^{*} X$, we shall not require that $\nabla^{g}$ be torsion free, that is, we shall not assume $\nabla^{g}$ is the Levi-Civita connection ${ }^{\mathrm{LC}} \nabla^{g}$ for the metric $g$. However, the relation between the Dirac operators defined by $(\rho, B, A)$ and two different $\mathrm{SO}(4)$ connections for the metric $g$ on $T^{*} X$ is easily determined:

Lemma 3.1. Let $X$ be an oriented four-manifold with Riemannian metric $g$ on $T^{*} X$, compatible Clifford map $\rho_{+}: T^{*} X \rightarrow \operatorname{Hom}_{\mathbb{C}}\left(W^{+}, W^{-}\right)$, unitary connection $B$ on $\operatorname{det} W^{+}$, and unitary connection $A$ on a Hermitian twoplane bundle $E$ over $X$. If ${ }^{j} \nabla^{g}, j=1,2$ are two $\mathrm{SO}(4)$ connections on $T^{*} X$ for the metric $g$, and ${ }^{j} \nabla$ are the unitary connections on $W$ induced by $\left(\rho,{ }^{j} \nabla^{g}, B\right)$, and ${ }^{j} \mathcal{D}_{A}$ are the Dirac operators defined by $\left(\rho,{ }^{j} \nabla, A\right)$, then

$$
{ }^{2} \mathcal{D}_{A}-{ }^{1} \mathcal{D}_{A}=\rho_{+}(\sigma) \otimes \mathrm{id}_{E},
$$

where

$$
\sigma:={ }^{2} \nabla-{ }^{1} \nabla \in \Omega^{1}\left(X, \mathfrak{s u}\left(W^{+}\right)\right)
$$

and $\rho_{+}(\sigma) \in \operatorname{Hom}_{\mathbb{C}}\left(W^{+}, W^{-}\right)$is defined by the contraction

$$
\rho_{+}: C^{\infty}\left(T^{*} X \otimes \operatorname{End}_{\mathbb{C}}\left(W^{+}\right)\right) \rightarrow C^{\infty}\left(\operatorname{Hom}_{\mathbb{C}}\left(W^{+}, W^{-}\right)\right) .
$$

Proof. Since ${ }^{2} \nabla$ and ${ }^{1} \nabla$ are unitary connections on $W^{+}$, then $\sigma={ }^{2} \nabla-{ }^{1} \nabla \epsilon$ $\Omega^{1}\left(X, \mathfrak{u}\left(W^{+}\right)\right)$, and as they both induce the connection $B$ on $\operatorname{det} W^{+}$, then $\operatorname{tr} \sigma=0$, so $\sigma \in \Omega^{1}\left(X, \mathfrak{s u}\left(W^{+}\right)\right)$. If $\left\{v^{i}\right\}$ is any local frame for $T^{*} X$ with dual frame $\left\{v_{i}\right\}$ for $T X$, defined by $v^{i}\left(v_{j}\right)=\delta_{i j}$, then the difference between the Dirac operators is given by

$$
\begin{aligned}
{ }^{2} \mathcal{D}_{A}-{ }^{1} \mathcal{D}_{A} & =\sum_{i=1}^{4} \rho_{+}\left(v^{i}\right)\left({ }^{2} \nabla_{A, v_{i}}-{ }^{1} \nabla_{A, v_{i}}\right) \\
& =\sum_{i=1}^{4} \rho_{+}\left(v^{i}\right) \sigma\left(v_{i}\right) \otimes \mathrm{id}_{E}=\rho_{+}(\sigma) \otimes \mathrm{id}_{E}
\end{aligned}
$$


with $\rho_{+}(\sigma) \in \operatorname{Hom}_{\mathbb{C}}\left(W^{+} W^{-}\right)$, as desired. Alternatively, the conclusion can be inferred from the expression for the local connection matrix one-forms for the connections ${ }^{j} \nabla$ given in the paragraphs preceding the statement of the lemma.

Lemma 3.1 allows us to write the Dirac operator in (1.1), defined by a Riemannian but not necessarily torsion-free connection $\nabla^{g}$ as the Dirac operator for ${ }^{\mathrm{LC}} \nabla^{g}$ plus an element $\rho_{+}(\theta)$ of $\operatorname{Hom}_{\mathbb{C}}\left(W^{+}, W^{-}\right)=\rho_{+}\left(T^{*} X \otimes\right.$ $\mathbb{C})$. Since we already include a perturbation term $\rho_{+}(\vartheta)$ in our definition of the $\mathrm{PU}(2)$ monopole equations, we may therefore assume at the conclusion of our transversality argument that the Dirac operator corresponds to the Levi-Civita connection for the Riemannian metric on $T^{*} X$ by absorbing the correction term $\rho_{+}(\theta)$ into $\rho_{+}(\vartheta)$. For the remainder of the article, however, the unitary connection on $W$ will be held fixed and so it induces a Riemannian but not always torsion-free connection on $T^{*} X$ via (3.6) as the metric on $T^{*} X$ is allowed to vary.

From (3.7) we obtain a family of Dirac operators parametrized by $C^{r}\left(\mathrm{GL}\left(T^{*} X\right)\right)$. Specifically, let $\mathcal{D}_{A, f}$ be the Dirac operator defined by the following data:

- Clifford map $\rho_{+} \circ f$ compatible with the metric $f^{*} g$ on $T^{*} X$, where $\rho_{+}$is a Clifford map compatible with the metric $g$, and $f \in$ $C^{\infty}\left(\mathrm{GL}\left(T^{*} X\right)\right)$,

- Unitary connection $\nabla$ on $W$,

- Unitary connection $A$ on a Hermitian two-plane bundle $E$.

If $\left\{v^{i}\right\}$ is an oriented local frame for $T^{*} X$ with dual frame $\left\{v_{i}\right\}$ for $T X$ defined by $v^{j}\left(v_{i}\right)=\delta_{i j}$, then

$$
\mathcal{D}_{A, f}=\sum_{i=1}^{4} \rho_{+}\left(f\left(v^{i}\right)\right) \nabla_{A, v_{i}} .
$$

is the corresponding family of Dirac operators.

The variation of the Dirac operator $\mathcal{D}_{A, f}$ induced from (3.5) is given by

$$
\left(D \mathcal{D}_{A}\right)_{f}(\delta f) \Phi=\sum_{i=1}^{4} \rho_{+}\left((\delta f) v^{i}\right) \nabla_{A, v_{i}} \Phi
$$


where $\Phi \in \Omega^{0}\left(W^{+} \otimes E\right)$. For a given Hermitian metric and unitary connection on $W^{+} \oplus W^{-}$, an orientation of $X$, and a Riemannian metric $g$ on $T^{*} X$, we then take our family of perturbed Dirac operators

$$
\mathcal{D}_{A, f, \vartheta}: \Omega^{0}\left(W^{+} \otimes E\right) \rightarrow \Omega^{0}\left(W^{-} \otimes E\right)
$$

to be

$$
\mathcal{D}_{A, f, \vartheta}:=\sum_{i=1}^{4} \rho_{+}\left(f\left(v^{i}\right)\right) \nabla_{A, v_{i}}+\rho_{+}(f(\vartheta)),
$$

for any unitary connection $A$ on $E$. The unitary connection $\nabla$ on $W^{+} \oplus W^{-}$ and Clifford map $\rho_{f,+}: T^{*} X \rightarrow \operatorname{Hom}_{\mathbb{C}}\left(W^{+}, W^{-}\right)$induces, via (3.6), an $\mathrm{SO}(4)$ (but necessarily torsion-free) connection on $T^{*} X$ for the metric $f^{*} g$.

Lemma 3.2. Continue the notation of this section. Then

$$
\begin{aligned}
(D \mathcal{D})_{A, f, \vartheta}(\delta A, \delta f, \delta \vartheta) \Phi & \\
& =\sum_{i=1}^{4} \rho_{+}\left((\delta f) v^{i}\right) \nabla_{A, v_{i}} \Phi+\rho_{+}(f(\delta A)) \Phi+\rho_{+}(f(\delta \vartheta)) \Phi .
\end{aligned}
$$

We note that a direct variation of the Dirac operator with respect to the Riemannian metric $g$ has been computed by Bourguignon and Gauduchon [7], using a rather different and more elaborate approach than the one considered here. Their computation, with some enhancements, was recently used by Maier to prove certain generic metrics results for Dirac operators on low-dimensional spin or $\operatorname{spin}^{c}$ manifolds [45]. It is not known at present whether there exists a purely 'generic metrics' transversality result for the Seiberg-Witten equations (analogous to the celebrated generic metrics theorem of Freed and Uhlenbeck [15], [23] for the anti-self-dual equation), although there have been some attempts in this direction [9].

Recall that the space $\operatorname{Met}(X)$ of all $C^{\infty}$ Riemannian metrics on $X$ is a contractible, open cone inside the space $S^{2}\left(T^{*} X\right)$ of symmetric, rank-two, contravariant tensor fields on $X$ and so, at any metric $g \in \operatorname{Met}(X)$, it has tangent space $T_{g} \operatorname{Met}(X)=S^{2}\left(T^{*} X\right)$. The technique employed by Bourguignon and Gauduchon constructs spinor bundles which depend implicitly on the Riemannian metric (as is standard [42]). Thus, in order to compute the differential of their family of Dirac operators parametrized by the space of metrics, the family must be pulled back to an equivalent family of operators acting on a fixed Hilbert space of sections of a fixed $\operatorname{spin}^{c}$ bundle. 
This is the technique employed by Bourguignon-Gauduchon and Maier; a similar one was used by the authors in our proof of Theorem 5.11 in [16]. It is important to note that the resulting family of operators in [7] is not necessarily a family of Dirac operators. In any event, their key result, namely [7, Theorem 21] (see also [45, Proposition 2.4]), could be used in place of the simpler variational formulas (3.8) which we shall employ in the sequel. The principal difference for our application would be that variations of $g$ alone do not span $\mathfrak{g l}(T X)$, so we would need to consider auxiliary variations of the Clifford map $\rho_{+}$by elements $f \in C^{\infty}\left(\mathrm{SO}_{g}\left(T^{*} X\right)\right)$.

\subsection{The parametrized moduli space of $P U(2)$ monopoles.}

Although the curvature equation in (1.1) will only play an auxiliary role in the proof of Theorem 4.1 (for example, through the local-to-global unique continuation result, Theorem 4.11, for reducible PU(2) monopoles), we will need a parametrized moduli space as a domain of definition for our infinitedimensional period map. In this section we define $\mathrm{PU}(2)$ monopole equations containing the full set of perturbations $(f, \tau, \vartheta)$ and define the corresponding parametrized moduli space.

We fix a $C^{\infty}$ Riemannian metric $g$ on $T^{*} X$ and set

$$
\mathcal{P}:=C^{r}\left(\mathrm{GL}\left(T^{*} X\right)\right) \oplus C^{r}\left(\Lambda^{1} \otimes \mathbb{C}\right) .
$$

As we remarked in $\S 2.1$, we could of course include the space $C^{r}\left(\mathrm{GL}\left(\Lambda^{+}\right)\right)$ of parameters $\tau$ in our definition of $\mathcal{P}$ in (3.10); we simply omit them for convenience here, as they play no role in $\S 3$ or $\S 4$, just as we omitted the parameters $f$ from the definition of $\mathcal{P}$ in $\S 2$.

For $f \in C^{r}\left(\mathrm{GL}\left(T^{*} X\right)\right)$, we let $\rho_{f}: T^{*} X \rightarrow \operatorname{End}_{\mathbb{C}}\left(W^{+} \oplus W^{-}\right)$be the Clifford map defined by $\rho_{f,+}$ and (3.2). The map $\rho_{f}$ is compatible with the Riemannian metric $f^{*} g$ on $T^{*} X$ in the sense of (3.3) and thus extends, in the usual way, to give an isomorphism of real three-plane bundles,

$$
\rho_{f}: \Lambda^{+, f^{*} g} \rightarrow \mathfrak{s u}\left(W^{+}\right) .
$$

Let $\left\{e^{i}\right\}$ be an oriented, $g$-orthonormal local frame for $T^{*} X$ and observe that $\left\{f\left(e^{i}\right)\right\}$ is an oriented, $f^{*} g$-orthonormal local frame for $T^{*} X$, so we have induced isomorphisms

$$
f^{-1}: \Lambda^{ \pm} \rightarrow \Lambda^{ \pm, f^{*} g}, \quad \omega \mapsto f^{-1}(\omega),
$$

taking $e^{1} \wedge e^{2}+e^{3} \wedge e^{4} \mapsto f\left(e^{1}\right) \wedge f\left(e^{2}\right)+f\left(e^{3}\right) \wedge f\left(e^{4}\right)$, and similarly for the remaining two elements of the standard local frame for $\Lambda^{+}:=\Lambda^{+, g}$ 
and the three elements of the local frame for $\Lambda^{-}:=\Lambda^{-, g}$. Note that if $P_{+}(g):=\frac{1}{2}\left(1+*_{g}\right): \Lambda^{2}=\Lambda^{+} \oplus \Lambda^{-} \rightarrow \Lambda^{+}$is the projection onto $g$-self-dual two-forms, then

$$
P_{+}\left(f^{*} g\right)=f^{-1} \circ P_{+}(g) \circ f: \Lambda^{2} \rightarrow \Lambda^{+, f^{*} g}
$$

is the projection onto $f^{*} g$-self-dual two-forms. (We find it convenient to use automorphisms $f$ of $T^{*} X$ to vary the metric $g$ on $T^{*} X$, while FreedUhlenbeck [23, pp. 51-52] vary the metric on $T X$ by automorphisms of $T X$.)

Suppose $\omega:=\sum_{i<j} \omega_{i j} e^{i} \wedge e^{j} \in \Omega^{2}(X, \mathbb{R})$. For any $\Phi \in C^{\infty}\left(W^{+}\right)$, we see that

$$
\begin{aligned}
\rho_{f}(\omega) \Phi & =\sum_{i<j} \omega_{i j} \rho_{f}\left(e^{i}\right) \rho_{f}\left(e^{j}\right) \Phi=\sum_{i<j} \omega_{i j} \rho\left(f\left(e^{i}\right)\right) \rho\left(f\left(e^{j}\right)\right) \Phi \\
& =\sum_{i<j} \omega_{i j} \rho\left(f\left(e^{i}\right) \wedge f\left(e^{j}\right)\right) \Phi=\rho\left(\sum_{i<j} \omega_{i j} f\left(e^{i} \wedge e^{j}\right)\right) \Phi \\
& =\rho(f(\omega)) \Phi=(\rho \circ f)(\omega) \Phi .
\end{aligned}
$$

Conversely, since $\left(\Phi \otimes \Phi^{*}\right)_{00} \in \Omega^{0}\left(\mathfrak{s u}\left(W^{+}\right)\right), \tau \in \Omega^{0}\left(\operatorname{GL}\left(\Lambda^{+}\right)\right)$, and $\tau_{f}:=$ $f^{-1} \circ \tau \circ f \in \Omega^{0}\left(\mathrm{GL}\left(\Lambda^{+, f^{*} g}\right)\right)$, we have

$$
\tau_{f} \rho_{f}^{-1}\left(\Phi \otimes \Phi^{*}\right)_{00}=f^{-1} \tau \rho^{-1}\left(\Phi \otimes \Phi^{*}\right)_{00} \in \Omega^{0}\left(\Lambda^{+, f^{*} g}\right) .
$$

For the metric $f^{*} g$ on $T^{*} X$, compatible Clifford map $\rho_{f}: \Lambda^{+, f^{*} g} \rightarrow \mathfrak{s u}\left(W^{+}\right)$, and compatible automorphism $\tau_{f}$ of $\Lambda^{+, f^{*} g}$, the curvature equation in (1.1) is given by

$$
P_{+}\left(f^{*} g\right) F_{A}-\tau_{f} \rho_{f}^{-1}\left(\Phi \otimes \Phi^{*}\right)_{00}=0 \in \Omega^{+, f^{*} g}(\mathfrak{s u}(E)),
$$

that is,

$$
\left(f^{-1} \circ P_{+}(g) \circ f\right)\left(F_{A}\right)-f^{-1} \rho^{-1} \tau\left(\Phi \otimes \Phi^{*}\right)_{00}=0,
$$

or equivalently,

$$
P_{+}(g) f\left(F_{A}\right)-\rho^{-1} \tau\left(\Phi \otimes \Phi^{*}\right)_{00}=0 \in \Omega^{+}(\mathfrak{s u}(E)) .
$$

We now define our ${ }^{\circ} \mathcal{G}_{E^{-}}$equivariant map

$$
\underline{\mathfrak{S}}: \mathcal{P} \times \tilde{\mathcal{C}}_{W, E} \rightarrow L_{k-1}^{2}\left(\Lambda^{+} \otimes(\mathfrak{s u}(E)) \oplus L_{k-1}^{2}\left(W^{-} \otimes E\right)\right)
$$


by setting

$$
\underline{\mathfrak{S}}(f, \vartheta, A, \Phi):=\left(\begin{array}{c}
P_{+}(g) f\left(F_{A}\right)-\tau \rho^{-1}\left(\Phi \otimes \Phi^{*}\right)_{00} \\
\mathcal{D}_{A, f} \Phi+\rho(f(\vartheta)) \Phi
\end{array}\right) .
$$

The group ${ }^{\circ} \mathcal{G}_{E}$ acts trivially on the space of perturbations $\mathcal{P}$ and so $\underline{\mathfrak{S}}^{-1}(0) /{ }^{\circ} \mathcal{G}_{E}$ is a subset of $\mathcal{P} \times \mathcal{C}_{W, E}$. We now let $\mathfrak{M}_{W, E}$ denote the parametrized (or universal) moduli space $\underline{\mathfrak{S}}^{-1}(0) /{ }^{\circ} \mathcal{G}_{E}$ for the augmented space $\mathcal{P}$ of perturbation parameters and let $\mathfrak{M}_{W, E}^{*, 0}:=\mathfrak{M}_{W, E} \cap\left(\mathcal{P} \times \mathcal{C}_{W, E}^{*, 0}\right)$.

\section{Period maps for Dirac operators.}

In $\S 2$ we proved that the moduli space of $\mathrm{PU}(2)$ monopoles is cut out transversely away from the loci of pairs $(A, \Phi)$ with either $A$ reducible or $\Phi$ rank less than or equal to one. In this section we prove the following analogue of Proposition 4.3.14 and Corollary 4.3.15 in [15] for the locus of pairs $(A, \Phi)$ with $A$ irreducible and $\Phi$ rank one but not identically zero:

Theorem 4.1. Let $X$ be a closed, oriented, smooth four-manifold with spin $^{c}$ structure $\left(\rho, W^{+}, W^{-}\right)$and Hermitian two-plane bundle $E$. Then there is a first-category subset $\mathcal{P}_{\mathrm{fc}}^{\infty} \subset \mathcal{P}^{\infty}$ such that for all $(f, \tau, \vartheta)$ in $\mathcal{P}^{\infty}-\mathcal{P}_{\mathrm{fc}}^{\infty}$ the following holds: The moduli space $M_{W, E}^{*, 0}(f, \tau, \vartheta)$ contains no $\mathrm{PU}(2)$ monopoles $(A, \Phi)$ with both $A$ irreducible and $\Phi$ rank one.

The argument we describe here only works for $\mathrm{PU}(2)$ monopoles $(A, \Phi)$ with $A$ irreducible. Thus, even for generic parameters $(f, \tau, \vartheta)$, the moduli space $M_{W, E}(f, \tau, \vartheta)$ will in general contain points $[A, \Phi]$ with $A$ reducible and $\Phi$ rank one and these will not necessarily be smooth points of $M_{W, E}(f, \tau, \vartheta)$. However, it is reassuring to note that the loci of reducible PU(2) monopoles - corresponding to moduli spaces of Seiberg-Witten monopoles - cannot be perturbed away by the argument we present here: the fact that the $\mathrm{PU}(2)$ monopole connections $A$ are irreducible is used in an essential way in the proof of the key Proposition 4.10.

Given Theorem 4.1, we can quickly dispose of the proof of Theorem 1.3.

Proof of Theorem 1.3, given Theorem 4.1. As we remarked at the beginning of $\S 2.1$ and $\S 3.2$, we may assume without loss of generality that the same Fréchet space of $C^{\infty}$ perturbation parameters,

$$
\mathcal{P}^{\infty}:=\Omega^{0}\left(\mathrm{GL}\left(T^{*} X\right)\right) \times \Omega^{0}\left(\mathrm{GL}\left(\Lambda^{+}\right)\right) \times \Omega^{0}\left(\Lambda^{1} \otimes \mathbb{C}\right),
$$


has been used for the proofs of both Theorem 2.2 and Theorem 4.1. By Theorem 2.2, there is a first-category subset $\mathcal{P}_{\text {fc }}^{\prime} \subset \mathcal{P}$ such that for all $p=(f, \tau, \vartheta) \in \mathcal{P}-\mathcal{P}_{\mathrm{fc}}^{\prime}$, the moduli space $M_{W, E}^{*, \mathrm{G}}(p)$ of irreducible, rank-two $\mathrm{PU}(2)$ monopoles is a smooth manifold of the expected dimension. On the other hand, by Theorem 4.1, there is a first-category subset $\mathcal{P}_{\mathrm{fc}}^{\prime \prime} \subset \mathcal{P}$ such that for all $p=(f, \tau, \vartheta) \in \mathcal{P}-\mathcal{P}_{\mathrm{fc}}^{\prime \prime}$, the moduli space $M_{W, E}^{*, 0}(p)$ contains no irreducible, rank-one $\mathrm{PU}(2)$ monopoles, that is, $M_{W, E}^{*, \downarrow}(p)=M_{W, E}^{*, 0}(p)$. Hence, for all $p \in \mathcal{P}-\mathcal{P}_{\mathrm{fc}}$, where $\mathcal{P}_{\mathrm{fc}}:=\mathcal{P}_{\mathrm{fc}}^{\prime} \cup \mathcal{P}_{\mathrm{fc}}^{\prime \prime}$, the moduli space $M_{W, E}^{*, 0}(p)$ is a smooth manifold of the expected dimension.

The remainder of $\S 4$ is taken up with the proof of Theorem 4.1 .

\subsection{Infinite-dimensional Grassmann manifolds.}

The period map described in $[15, \S 4.3 .3 \& 4.3 .5]$ takes values in the Grassmann manifold $\mathbb{G}\left(H^{2}(X ; \mathbb{R})\right)$ of $b^{-}(X)$-dimensional subspaces of $H^{2}(X ; \mathbb{R})$. We shall need to consider an infinite-dimensional version of this construction, so it is convenient at this point to recall some properties of infinitedimensional Grassmann manifolds [1, §3.1.8], [34, §1.1].

If $\mathbf{E}$ is a complex Banach space then the Grassmann manifold $\mathbb{G}(\mathbf{E})$ is the set of splitting linear subspaces of $\mathbf{E}$ and is a complex-analytic manifold with tangent spaces

$$
T_{K} \mathbb{G}(\mathbf{E})=\operatorname{Hom}_{\mathbb{C}}(K, \mathbf{E} / K)
$$

Recall that a closed, linear subspace $K \subset \mathbf{E}$ splits if $K$ has a closed complement $K^{\prime} \subset \mathbf{E}$ such that $\mathbf{E} \simeq K \times K^{\prime}$ and that any finite-dimensional subspace splits. For each integer $\kappa \geq 1$, let

$$
\mathbb{G}_{\kappa}(\mathbf{E}):=\{K \subset \mathbf{E}: K \text { is a } \kappa \text {-dimensional subspace of } \mathbf{E}\},
$$

and set $\mathbb{P}(\mathbf{E})=\mathbb{G}_{1}(\mathbf{E})$. The spaces $\mathbb{G}_{\kappa}(\mathbf{E})$ are connected submanifolds of $\mathbb{G}(\mathbf{E})$.

The Grassmann manifolds $\mathbb{P}(\mathbf{E})$ and $\mathbb{G}_{\kappa}(\mathbf{E})$ define a locus of incident planes (or flag manifold) $\mathbb{F}_{\kappa}(\mathbf{E}) \subset \mathbb{P}(\mathbf{E}) \times \mathbb{G}_{\kappa}(\mathbf{E})$ by analogy with the usual construction in the finite-dimensional case [27]:

$$
\mathbb{F}_{\kappa}(\mathbf{E}):=\left\{(\ell, K) \in \mathbb{P}(\mathbf{E}) \times \mathbb{G}_{\kappa}(\mathbf{E}): \ell \subset K\right\}
$$


The locus $\mathbb{F}_{\kappa}(\mathbf{E})$ is a smooth manifold with tangent spaces

$$
\begin{aligned}
& T_{(\ell, K)} \mathbb{F}_{\kappa}(\mathbf{E}) \\
& \quad=\left\{(\eta, \varphi) \in \operatorname{Hom}_{\mathbb{C}}(\ell, \mathbf{E} / \ell) \oplus \operatorname{Hom}_{\mathbb{C}}(K, \mathbf{E} / K):\left.\varphi\right|_{\ell}=\eta \quad(\bmod K)\right\} .
\end{aligned}
$$

Given a smooth submanifold $\mathfrak{X} \subset \mathbb{P}(\mathbf{E})$, we define a locus $\mathbb{I}_{\kappa}(\mathfrak{X}) \subset \mathbb{G}_{\kappa}(\mathbf{E})$ of incident $\kappa$-planes by setting

$$
\mathbb{I}_{\kappa}(\mathfrak{X}):=\pi_{2}\left(\pi_{1}^{-1}(\mathfrak{X})\right) \subset \mathbb{G}_{\kappa}(\mathbf{E}),
$$

where $\pi_{1}, \pi_{2}$ are the projections from the flag manifold onto the first and second factors:

$$
\begin{gathered}
\pi_{1} \swarrow \quad \mathbb{F}_{\kappa}(\mathbf{E}) \\
\mathbb{P}(\mathbf{E}) \\
\quad \mathbb{G}_{\kappa}(\mathbf{E})
\end{gathered}
$$

We then have the following straightforward observations:

Claim 4.2. The map

$$
\pi_{1}: \mathbb{F}_{\kappa}(\mathbf{E}) \rightarrow \mathbb{P}(\mathbf{E}), \quad(\ell, K) \mapsto \ell
$$

is a submersion.

Proof. From the description of $T_{(\ell, K)} \mathbb{F}_{\kappa}(\mathbf{E})$ in (4.1) and of $T_{\ell} \mathbb{P}(\mathbf{E})$ a.s $\operatorname{Hom}_{\mathbb{C}}(\ell, \mathbf{E} / \ell)$, we see that for any $(\ell, K) \in \mathbb{F}_{\kappa}(\mathbf{E})$, the differential (which is again projection onto the first factor),

$$
\left(D \pi_{1}\right)_{(\ell, K)}: T_{(\ell, K)} \mathbb{F}_{\kappa}(\mathbf{E}) \rightarrow T_{\ell} \mathbb{P}(\mathbf{E}), \quad(\eta, \varphi) \mapsto \eta,
$$

is surjective. Indeed, if $\eta \in \operatorname{Hom}_{\mathbb{C}}(\ell, \mathbf{E} / \ell)$, then we can choose $\varphi \in$ $\operatorname{Hom}_{\mathbb{C}}(K, \mathbf{E} / K)$ such that $\varphi(\ell)=\eta$ and $\varphi$ is defined arbitrarily on $K / \ell$, so $\left(D \pi_{1}\right)_{(\ell, K)}(\eta, \varphi)=\eta$.

Hence, the locus $\tilde{\mathbb{I}}_{\kappa}(\mathfrak{X}):=\pi_{1}^{-1}(\mathfrak{X}) \subset \mathbb{F}_{\kappa}(\mathbf{E})$ is a smooth submanifold with tangent spaces

$$
T_{(\ell, K)} \tilde{\mathbb{I}}_{\kappa}(\mathfrak{X})=\left\{(\eta, \varphi) \in T_{\ell} \mathfrak{X} \oplus \operatorname{Hom}_{\mathbb{C}}(K, \mathbf{E} / K):\left.\varphi\right|_{\ell}=\eta \quad(\bmod K)\right\},
$$

where we view $T_{\ell} \mathfrak{X}$ as a subspace of $\operatorname{Hom}_{\mathbb{C}}(\ell, \mathbf{E} / \ell)$, and codimension

$$
\operatorname{codim}\left(\tilde{\mathbb{I}}_{\kappa}(\mathfrak{X}) ; \mathbb{F}_{\kappa}(\mathbf{E})\right)=\operatorname{codim}(\mathfrak{X} ; \mathbb{P}(\mathbf{E})) .
$$

In the same vein, we have: 
Claim 4.3. The map

$$
\pi_{2}: \mathbb{F}_{\kappa}(\mathbf{E}) \rightarrow \mathbb{G}_{\kappa}(\mathbf{E}), \quad(\ell, K) \mapsto K,
$$

is a submersion.

Proof. We see that the differential (which is again projection onto the second factor)

$$
\left(D \pi_{2}\right)_{(\ell, K)}: T_{(\ell, K)} \mathbb{F}_{\kappa}(\mathbf{E}) \rightarrow T_{K} \mathbb{G}_{\kappa}(\mathbf{E}) \quad(\eta, \varphi) \mapsto \varphi,
$$

is surjective from the description of $T_{(\ell, K)} \mathbb{F}_{\kappa}(\mathbf{E})$ in (4.1) and of $T_{K} \mathbb{G}_{\kappa}(\mathbf{E})$ as $\operatorname{Hom}_{\mathbb{C}}(K, \mathbf{E} / K)$. Indeed, if $\varphi \in \operatorname{Hom}_{\mathbb{C}}(K, \mathbf{E} / K)$, then we can choose $\eta \in \operatorname{Hom}_{\mathbb{C}}(\ell, \mathbf{E} / \ell)$ by setting $\eta=\left.\varphi\right|_{\ell}$, so $\left(D \pi_{2}\right)_{(\ell, K)}(\eta, \varphi)=\varphi$.

Over each point $K \in \mathbb{G}_{\kappa}(\mathbf{E})$, the projection (4.3) has fibers

$$
\pi_{2}^{-1}(K)=\{\ell \in \mathbb{P}(\mathbf{E}): \ell \subset K\}=\mathbb{P}(K),
$$

given by finite-dimensional, complex projective spaces $\mathbb{P}(K) \simeq \mathbb{P}^{\kappa-1}$.

The image $\mathbb{I}_{\kappa}(\mathfrak{X}) \subset \mathbb{G}_{\kappa}(\mathbf{E})$ of $\tilde{\mathbb{I}}_{\kappa}(\mathfrak{X}) \subset \mathbb{F}_{\kappa}(\mathbf{E})$ under the projection map $\pi_{2}: \mathbb{P}(\mathbf{E}) \times \mathbb{G}_{\kappa}(\mathbf{E}) \rightarrow \mathbb{G}_{\kappa}(\mathbf{E})$ is not necessarily a smooth submanifold. For this reason, we restrict our attention henceforth to the smooth submanifold $\tilde{\mathbb{I}}_{\kappa}(\mathfrak{X}) \subset \mathbb{F}_{\kappa}(\mathbf{E})$.

Remark 4.4. If the Banach space $\mathbf{E}$ were finite-dimensional, so $\mathbf{E}=\mathbb{C}^{n+1}$, $\mathbb{P}(\mathbf{E})=\mathbb{P}^{n}$, and $\mathbb{G}_{\kappa}(\mathbf{E})=\mathbb{G}(\kappa, n+1)$, and $\mathfrak{X} \subset \mathbb{P}^{n}$ were a complex projective variety, then the incidence locus $\mathbb{I}_{\kappa}(\mathfrak{X}) \subset \mathbb{G}(\kappa, n+1)$ would be a complex projective subvariety with smooth locus $\mathbb{I}_{\kappa}(\mathfrak{X})_{\text {sm }}$ of codimension [27, Example 16.6]

$$
\operatorname{codim}\left(\mathbb{I}_{\kappa}(\mathfrak{X})_{\mathrm{sm}} ; \mathbb{G}(\kappa, n+1)\right)=\operatorname{codim}\left(\mathfrak{X}_{\mathrm{sm}} ; \mathbb{P}^{n}\right)-(\kappa-1),
$$

where $\mathbb{I}_{\kappa}(\mathfrak{X})$ has a standard stratification by virtue of its status as a complex projective variety. When $\mathbf{E}$ is an infinite-dimensional Banach space, as in our application, it would suffice for our purposes to show that the image $\mathbb{I}_{\kappa}(\mathfrak{X})$ of $\tilde{\mathbb{I}}_{\kappa}(\mathfrak{X})=\pi_{1}^{-1}(\mathfrak{X})$ under the projection to $\mathbb{G}_{\kappa}(\mathbf{E})$, is contained in a countable union of smooth submanifolds of $\mathbb{G}_{\kappa}(\mathbf{E})$ with infinite codimension. 


\subsection{Spaces of Fredholm operators.}

For a non-negative integer $n$, let $\operatorname{Fred}_{n}\left(\mathbf{E}^{+}, \mathbf{E}^{-}\right)$denote the open subset of the Banach space of bounded operators, $\operatorname{Hom}_{\mathbb{C}}\left(\mathbf{E}^{+}, \mathbf{E}^{-}\right)$, consisting of bounded, index- $n$ Fredholm operators, where $\mathbf{E}^{+}:=L_{k}^{2}\left(W^{+} \otimes E\right)$ and $\mathbf{E}^{-}:=L_{k-1}^{2}\left(W^{-} \otimes E\right)$ [34]. While the direct analogue of the period map of [11], [15], [23] would use the infinite-dimensional Grassmann manifold $\mathbb{G}\left(\mathbf{E}^{+}\right)$as a target space, this is somewhat less suitable for our purposes since the dimension of $\operatorname{Ker} \mathcal{D}_{A, f, \vartheta}$ might jump due to spectral flow as the point $[A, \Phi, f, \vartheta]$ varies in $\mathcal{C}_{W, E}^{*, 0} \times \mathcal{P}$; consequently, the assignment $(A, \Phi, f, \vartheta) \mapsto \operatorname{Ker} \mathcal{D}_{A, f, \vartheta}$ might not define a smooth map from $\tilde{\mathcal{C}}_{W, E}^{*, 0} \times \mathcal{P}$ to $\mathbb{G}\left(\mathbf{E}^{+}\right)$. However, the assignment $(A, \Phi, f, \vartheta) \mapsto \operatorname{Ker} \mathcal{D}_{A, f, \vartheta}$ does give a smooth map from $\tilde{\mathcal{C}}_{W, E}^{*, 0} \times \mathcal{P}$ to $\operatorname{Fred}_{n}\left(\mathbf{E}^{+}, \mathbf{E}^{-}\right)$. Indeed, a map of this form is used by Maier [45] (with domain $\operatorname{Met}(X)$, the space of all $C^{r}$ Riemannian metrics on $\left.T^{*} X\right)$.

Passing temporarily to a more general setting, let $\mathbf{E}^{+}, \mathbf{E}^{-}$be complex Hilbert spaces. The subsets

$$
\operatorname{Fred}_{\kappa, n}\left(\mathbf{E}^{+}, \mathbf{E}^{-}\right):=\left\{B \in \operatorname{Fred}_{n}\left(\mathbf{E}^{+}, \mathbf{E}^{-}\right): \operatorname{dim}_{\mathbb{C}} \operatorname{Ker} B=\kappa\right\}
$$

are locally-closed submanifolds of $\operatorname{Hom}_{\mathbb{C}}\left(\mathbf{E}^{+}, \mathbf{E}^{-}\right)$. The normal bundle $N \operatorname{Fred}_{\kappa, n}\left(\mathbf{E}^{+}, \mathbf{E}^{-}\right)$of the submanifold $\operatorname{Fred}_{\kappa, n}\left(\mathbf{E}^{+}, \mathbf{E}^{-}\right)$relative to $\operatorname{Fred}_{n}\left(\mathbf{E}^{+}, \mathbf{E}^{-}\right)$, and so $\operatorname{Hom}_{\mathbb{C}}\left(\mathbf{E}^{+}, \mathbf{E}^{-}\right)$, has fiber

$$
N_{B} \operatorname{Fred}_{\kappa, n}\left(\mathbf{E}^{+}, \mathbf{E}^{-}\right)=\operatorname{Hom}_{\mathbb{C}}(\operatorname{Ker} B, \operatorname{Coker} B)
$$

over a point $B \in \operatorname{Fred}_{\kappa, n}\left(\mathbf{E}^{+}, \mathbf{E}^{-}\right)$. The submanifold $\operatorname{Fred}_{\kappa, n}\left(\mathbf{E}^{+}, \mathbf{E}^{-}\right)$thus has complex codimension $\kappa(\kappa-n)$ in $\operatorname{Fred}_{n}\left(\mathbf{E}^{+}, \mathbf{E}^{-}\right)$and we have a smooth stratification

$$
\operatorname{Fred}_{n}\left(\mathbf{E}^{+}, \mathbf{E}^{-}\right)=\bigcup_{\kappa \geq n} \operatorname{Fred}_{\kappa, n}\left(\mathbf{E}^{+}, \mathbf{E}^{-}\right)
$$

with top stratum $\operatorname{Fred}_{n, n}\left(\mathbf{E}^{+}, \mathbf{E}^{-}\right)$.

Associated with each $\operatorname{Fred}_{\kappa, n}\left(\mathbf{E}^{+}, \mathbf{E}^{-}\right)$, we have a 'flag manifold'

$$
\begin{aligned}
\operatorname{Flag}_{\kappa, n}\left(\mathbf{E}^{+}, \mathbf{E}^{-}\right): & =\left\{(\ell, B) \in \mathbb{P}\left(\mathbf{E}^{+}\right) \times \operatorname{Fred}_{\kappa, n}\left(\mathbf{E}^{+}, \mathbf{E}^{-}\right): \ell \in \operatorname{Ker} B\right\} \\
& \subset \mathbb{P}\left(\mathbf{E}^{+}\right) \times \operatorname{Fred}_{\kappa, n}\left(\mathbf{E}^{+}, \mathbf{E}^{-}\right) .
\end{aligned}
$$

The corresponding projection

$$
\pi: \operatorname{Flag}_{\kappa, n}\left(\mathbf{E}^{+}, \mathbf{E}^{-}\right) \rightarrow \operatorname{Fred}_{\kappa, n}\left(\mathbf{E}^{+}, \mathbf{E}^{-}\right), \quad(\ell, B) \mapsto B,
$$


has fiber over a point $B \in \operatorname{Fred}_{\kappa, n}\left(\mathbf{E}^{+}, \mathbf{E}^{-}\right)$,

$$
\pi^{-1}(B)=\left\{\ell \in \mathbb{P}\left(\mathbf{E}^{+}\right): \ell \subset \operatorname{Ker} B\right\}=\mathbb{P}(\operatorname{Ker} B),
$$

given by a finite-dimensional, complex projective space $\mathbb{P}(\operatorname{Ker} B) \simeq \mathbb{P}^{\kappa-1}$.

Furthermore, we have smooth maps

$$
\pi: \operatorname{Fred}_{\kappa, n}\left(\mathbf{E}^{+}, \mathbf{E}^{-}\right) \rightarrow \mathbb{G}_{\kappa}\left(\mathbf{E}^{+}\right), \quad B \mapsto \operatorname{Ker} B,
$$

which are easily seen to be submersions:

Lemma 4.5. The canonical map $\pi: \operatorname{Fred}_{\kappa, n}\left(\mathbf{E}^{+}, \mathbf{E}^{-}\right) \rightarrow \mathbb{G}_{\kappa}\left(\mathbf{E}^{+}\right)$is a submersion.

Proof. We first compute the differential $(D \pi)_{B}$ of $\pi$ at a point $B \in$ $\operatorname{Fred}_{\kappa, n}\left(\mathbf{E}^{+}, \mathbf{E}^{-}\right)$. Let $B_{t}$ be a smooth path in $\operatorname{Fred}_{\kappa, n}\left(\mathbf{E}^{+}, \mathbf{E}^{-}\right)$through $B_{0}:=B$. We then have a smooth path of operators $B_{t}^{\dagger} B_{t} \in \operatorname{End}_{\mathbb{C}}\left(\mathbf{E}^{+}\right)$ giving isomorphisms $B_{t}^{\dagger} B_{t}:\left(\operatorname{Ker} B_{t}\right)^{\perp} \rightarrow\left(\operatorname{Ker} B_{t}\right)^{\perp}$, where $\operatorname{Ran} B_{t}^{\dagger}=$ $\left(\operatorname{Ker} B_{t}\right)^{\perp}$ since $B_{t}$ is Fredholm and thus has closed range. Let

$$
G_{t}:=\left(B_{t}^{\dagger} B_{t}\right)^{-1} B_{t}^{\dagger} \in \operatorname{Hom}_{\mathbb{C}}\left(\mathbf{E}^{-}, \mathbf{E}^{+}\right)
$$

be the corresponding smooth path of Green's operators and let

$$
\Pi_{t}:=\operatorname{id}_{\mathbf{E}^{+}}-G_{t} B_{t} \in \operatorname{End}_{\mathbb{C}}\left(\mathbf{E}^{+}\right)
$$

be the resulting smooth path of projections from $\mathbf{E}^{+}$onto $\operatorname{Ker} B_{t}$. Differentiating this path yields

$$
\begin{aligned}
\frac{d \Pi_{t}}{d t} & =-\frac{d G_{t}}{d t} B_{t}-G_{t} \frac{d B_{t}}{d t} \\
& =G_{t} \frac{d B_{t}}{d t} G_{t} B_{t}-G_{t} \frac{d B_{t}}{d t} .
\end{aligned}
$$

So, if $\delta B:=\left.\left(d B_{t} / d t\right)\right|_{t=0}$ and $G:=G_{0}$ and $\Pi:=\Pi_{0}$, we have

$$
\begin{aligned}
(D \pi)_{B} & =\left.\frac{d \Pi_{t}}{d t}\right|_{t=0}=G(\delta B)\left(G B-\mathrm{id}_{\mathbf{E}^{+}}\right) \\
& =-G(\delta B) \Pi \in \operatorname{Hom}_{\mathbb{C}}\left(\operatorname{Ker} B,(\operatorname{Ker} B)^{\perp}\right),
\end{aligned}
$$

with $\delta B \in T_{B} \operatorname{Fred}_{\kappa, n}\left(\mathbf{E}^{+}, \mathbf{E}^{-}\right)$. Any operator $A \in \operatorname{Hom}_{\mathbb{C}}\left(\mathbf{E}^{+}, \mathbf{E}^{-}\right)$may be decomposed as a block-matrix

$$
A=\left(\begin{array}{ll}
A_{11} & A_{12} \\
A_{21} & A_{22}
\end{array}\right):(\operatorname{Ker} B)^{\perp} \oplus \operatorname{Ker} B \rightarrow \operatorname{Ran} B \oplus(\operatorname{Ran} B)^{\perp},
$$


with $A_{22} \in \operatorname{Hom}_{\mathbb{C}}\left(\operatorname{Ker} B,(\operatorname{Ran} B)^{\perp}\right)$, the normal space to $T_{B} \operatorname{Fred}_{\kappa, n}\left(\mathbf{E}^{+}, \mathbf{E}^{-}\right)$ in $\operatorname{Hom}_{\mathbb{C}}\left(\mathbf{E}^{+}, \mathbf{E}^{-}\right)$. Then,

$$
A_{12} \in \operatorname{Hom}_{\mathbb{C}}(\operatorname{Ker} B, \operatorname{Ran} B) \subset T_{B} \operatorname{Fred}_{\kappa, n}\left(\mathbf{E}^{+}, \mathbf{E}^{-}\right),
$$

where $A_{12}$ is regarded as an operator in $\operatorname{Hom}_{\mathbb{C}}\left(\mathbf{E}^{+}, \mathbf{E}^{-}\right)$by extending by zero, so $A_{12}:=0$ on $(\operatorname{Ker} B)^{\perp}$. Indeed, $A_{12}$ is a compact operator (since $\operatorname{dim} \operatorname{Ker} B<\infty$, so $A_{12}$ is finite rank) and thus $\operatorname{ind}\left(B+A_{12}\right)=\operatorname{ind} B$. Moreover, $\operatorname{Ran}\left(B+A_{12}\right)=\operatorname{Ran} B$, so $\operatorname{Coker}\left(B+A_{12}\right)=$ Coker $B$ and thus $\operatorname{dim} \operatorname{Coker}\left(B+A_{12}\right)=\operatorname{dim}$ Coker $B$ implies $\operatorname{dim} \operatorname{Ker}\left(B+A_{12}\right)=\operatorname{dim} \operatorname{Ker} B=$ $\kappa$. Hence, $B_{t}:=B+t A_{12} \in \operatorname{Fred}_{\kappa, n}\left(\mathbf{E}^{+}, \mathbf{E}^{-}\right)$for all $t \in \mathbb{R}$ and, in particular, $\left.\left(d B_{t} / d t\right)\right|_{t=0}=A_{12} \in T_{B} \operatorname{Fred}_{\kappa, n}\left(\mathbf{E}^{+}, \mathbf{E}^{-}\right)$. Since $G: \operatorname{Ran} B \rightarrow(\operatorname{Ker} B)^{\perp}$ is an isomorphism and we may choose any $\delta B \in \operatorname{Hom}_{\mathbb{C}}(\operatorname{Ker} B, \operatorname{Ran} B)$, we see that

$$
\begin{aligned}
\operatorname{Ran}(D \pi)_{B} & =\left\{G(\delta B) \Pi: \delta B \in T_{B} \operatorname{Fred}_{\kappa, n}\left(\mathbf{E}^{+}, \mathbf{E}^{-}\right)\right\} \\
& =\operatorname{Hom}_{\mathbb{C}}\left(\operatorname{Ker} B,(\operatorname{Ker} B)^{\perp}\right)=T_{\operatorname{Ker} B} \mathbb{G}\left(\mathbf{E}^{+}\right),
\end{aligned}
$$

and so $(D \pi)_{B}$ is surjective, as desired.

Consequently, we have:

Lemma 4.6. The space $\operatorname{Flag}_{\kappa, n}\left(\mathbf{E}^{+}, \mathbf{E}^{-}\right)$is a smooth submanifold of

$$
\mathbb{P}\left(\mathbf{E}^{+}\right) \times \operatorname{Fred}_{\kappa, n}\left(\mathbf{E}^{+}, \mathbf{E}^{-}\right)
$$

and the canonical map $\pi: \operatorname{Flag}_{\kappa, n}\left(\mathbf{E}^{+}, \mathbf{E}^{-}\right) \rightarrow \mathbb{F}_{\kappa}\left(\mathbf{E}^{+}\right)$is a submersion.

Proof. Lemma 4.5 implies that the projection,

$$
\pi: \mathbb{P}\left(\mathbf{E}^{+}\right) \times \operatorname{Fred}_{\kappa, n}\left(\mathbf{E}^{+}, \mathbf{E}^{-}\right) \rightarrow \mathbb{P}\left(\mathbf{E}^{+}\right) \times \mathbb{G}_{\kappa}\left(\mathbf{E}^{+}\right), \quad(\ell, B) \mapsto(\ell, \operatorname{Ker} B),
$$

is a submersion. So, as $\mathbb{F}_{\kappa}\left(\mathbf{E}^{+}\right)$is a smooth submanifold of $\mathbb{P}\left(\mathbf{E}^{+}\right) \times \mathbb{G}_{\kappa}\left(\mathbf{E}^{+}\right)$, we see that the preimage

$$
\operatorname{Flag}_{\kappa, n}\left(\mathbf{E}^{+}, \mathbf{E}^{-}\right)=\pi^{-1}\left(\mathbb{F}_{\kappa}\left(\mathbf{E}^{+}\right)\right)
$$

is a smooth submanifold of $\mathbb{P}\left(\mathbf{E}^{+}\right) \times \operatorname{Fred}_{\kappa, n}\left(\mathbf{E}^{+}, \mathbf{E}^{-}\right)$and that the restriction of the projection map is a submersion. 


\subsection{The locus of rank-one sections.}

With the general setting of $\S 4.1$ and $\S 4.2$ at hand, we can now describe the locus of rank-one sections in $L_{k-1}^{2}\left(W^{+} \otimes E\right)$ and the Dirac-operator period map which is to be transverse, in a suitable sense, to this locus.

Assume $k \geq 4$, so that $\mathbf{E}^{+}=L_{k-1}^{2}\left(W^{+} \otimes E\right)$ is contained in $C^{0}\left(W^{+} \otimes E\right)$. Since $C^{0}\left(W^{+} \otimes E\right)=C^{0}\left(\operatorname{Hom}_{\mathbb{C}}\left(W^{+, *}, E\right)\right)$, we have a determinant map

$$
\operatorname{det}: C^{0}\left(W^{+} \otimes E\right) \rightarrow C^{0}\left(\operatorname{det} E \otimes \operatorname{det} W^{+}\right), \quad \Phi \mapsto \operatorname{det} \Phi,
$$

recalling that $\operatorname{det} W^{+}=\Lambda^{2}\left(W^{+}\right)$and $\operatorname{det} E=\Lambda^{2}(E)$. If $\left\{\xi_{1}, \xi_{2}\right\}$ is a local unitary frame for $E$ and $\left\{\phi_{1}, \phi_{2}\right\}$ is a local unitary frame for $W^{+}$, with dual coframe $\left\{\phi_{1}^{*}, \phi_{2}^{*}\right\}$ for $W^{+, *}$ defined by the Hermitian metric via $\phi_{j}^{*}:=$ $\left\langle\cdot, \phi_{j}\right\rangle$, then the section $\operatorname{det} \Phi$ of $\operatorname{det} E \otimes \operatorname{det} W^{+} \simeq \operatorname{Hom}_{\mathbb{C}}\left(\left(\operatorname{det} W^{+}\right)^{*}, \operatorname{det} E\right)$ is defined in the usual way with respect to these frames by

$$
\Phi\left(\phi_{1}^{*}\right) \wedge \Phi\left(\phi_{2}^{*}\right)=(\operatorname{det} \Phi) \phi_{1}^{*} \wedge \phi_{2}^{*} \in \mathbb{C} \xi_{1} \wedge \xi_{2} .
$$

A section $\Phi \in C^{0}\left(W^{+} \otimes E\right)$ then has rank one if $\operatorname{det} \Phi \equiv 0$ and $\Phi \not \equiv 0$ on $X$. Locally, any rank-one section $\Phi \in C^{0}\left(W^{+} \otimes E\right)$ may be written as $\Phi=\phi \otimes \xi$, for local sections $\phi \in C^{0}\left(W^{+}\right)$and $\xi \in C^{0}(E)$, though it is generally not possible to write a rank-one section globally in this form.

We can now conveniently define the 'rank-one locus' $\mathfrak{X} \subset \mathbb{P}\left(\mathbf{E}^{+}\right)$by

$$
\mathfrak{X}:=\left\{[\Phi] \in \mathbb{P}\left(\mathbf{E}^{+}\right): \operatorname{det} \Phi=0\right\},
$$

where $[\Phi]$ denotes the line $\mathbb{C} \cdot \Phi \subset \mathbf{E}^{+}$. Unless we impose further conditions on the Riemannian four-manifold $X$ and the bundles $W^{+}$and $E-$ such as requiring them to be real analytic - the locus of rank-one sections will not be a smooth subvariety of $\mathbb{P}\left(\mathbf{E}^{+}\right)$. However, as we shall see in the sequel, it suffices to work with the locus $\mathfrak{X}_{\text {sm }} \subset \mathfrak{X}$ of smooth points of $\mathfrak{X}$. The following lemma provides a simple criterion for point $[\Phi] \in \mathfrak{X}$ to be smooth.

Lemma 4.7. Suppose $[\Phi] \in \mathbb{P}\left(\mathbf{E}^{+}\right)$is point in the zero locus $\mathfrak{X}=\operatorname{det}^{-1}(0)$ such that $\{\Phi \neq 0\}$ is a dense open subset of $X$. Then the map $\operatorname{det}: C^{0}(E \otimes$ $\left.W^{+}\right) \rightarrow C^{0}\left(\operatorname{det} E \otimes \operatorname{det} W^{+}\right)$vanishes transversely at $\Phi$ and $[\Phi]$ is a smooth point of $\mathfrak{X}$. The tangent space $T_{[\Phi]} \mathfrak{X}$ has both infinite dimension and infinite codimension in $T_{[\Phi]} \mathbb{P}\left(\mathbf{E}^{+}\right)$, so

$$
\operatorname{codim}\left(\mathfrak{X}_{\mathrm{sm}} ; \mathbb{P}\left(\mathbf{E}^{+}\right)\right)=\infty .
$$


Proof. Choose a local unitary frame $\left\{\xi_{1}, \xi_{2}\right\}$ for $E$ and a local unitary frame $\left\{\phi_{1}, \phi_{2}\right\}$ for $W^{+}$, with dual coframe $\left\{\phi_{1}^{*}, \phi_{2}^{*}\right\}$ for $W^{+, *}$ defined by the Hermitian metric on $W^{+}$via $\phi_{j}^{*}:=\left\langle\cdot, \phi_{j}\right\rangle$. With respect to these local frames over an open subset $U \subset X$, the section $\Phi$ and determinant map are represented by

$$
\begin{aligned}
\operatorname{det}: C^{\infty}(U, \mathfrak{g l}(2, \mathbb{C})) & \rightarrow C^{\infty}(U, \mathbb{C}), \\
\Phi=\left(\begin{array}{ll}
\varphi_{11} & \varphi_{12} \\
\varphi_{21} & \varphi_{22}
\end{array}\right) & \mapsto \operatorname{det} \Phi=\varphi_{11} \varphi_{22}-\varphi_{12} \varphi_{21},
\end{aligned}
$$

with differential

$$
(D \operatorname{det})_{\Phi}(\delta \Phi)=\left(\delta \varphi_{11}\right) \varphi_{22}+\varphi_{11}\left(\delta \varphi_{22}\right)-\left(\delta \varphi_{12}\right) \varphi_{21}-\varphi_{12}\left(\delta \varphi_{21}\right)
$$

where

$$
\delta \Phi:=\left(\begin{array}{ll}
\delta \varphi_{11} & \delta \varphi_{12} \\
\delta \varphi_{21} & \delta \varphi_{22}
\end{array}\right) \in C^{\infty}(U, \mathfrak{g l}(2, \mathbb{C})) .
$$

Since $\{\Phi \neq 0\}$ is a dense open subset of $X$, the union of the complements of each of the zero-sets of the functions $\varphi_{i j}$ is a dense open subset of $U$. Now suppose that $\eta \in C^{0}\left(\operatorname{det} E \otimes \operatorname{det} W^{+}\right)$lies in $\operatorname{Coker}(D \operatorname{det})_{\Phi}$, so $\left((D \text { det })_{\Phi}(\delta \Phi), \eta\right)_{L^{2}(X)}=0$ for all $\delta \Phi \in C^{0}\left(W^{+} \otimes E\right)$. Since $\{\Phi \neq 0\}$ is a dense open subset of $U$, we have $\eta \equiv 0$ on $U$ and, as $U$ was an arbitrary open subset of $X$, we have $\eta \equiv 0$ on $X$.

Aronszajn's theorem then yields the following useful consequence of the preceding lemma.

Corollary 4.8. If $[A, \Phi, f, \vartheta]$ is a point in $\mathfrak{M}_{W, E}^{*, 0}$ such that $\operatorname{det} \Phi=0$, then $[\Phi]$ is a smooth point of the zero locus $\mathfrak{X}=\operatorname{det}^{-1}(0) \subset \mathbb{P}\left(\mathbf{E}^{+}\right)$, that is,

$$
\pi\left(\tilde{\mathfrak{M}}_{W, E}^{*, 0}\right) \subset \mathfrak{X}_{\mathrm{sm}}
$$

where $\pi: \tilde{\mathfrak{M}}_{W, E}^{*, 0} \rightarrow \mathbb{P}\left(\mathbf{E}^{+}\right)$is the projection.

Proof. The hypotheses imply that $\Phi \in \operatorname{Ker} \mathcal{D}_{A, f, \vartheta}$. Thus, Aronszajn's theorem [5] implies that $\{\Phi \neq 0\}$ is a dense open subset of $X$, so $[\Phi]$ is a smooth point of $\mathfrak{X}$ by Lemma 4.7 .

For each integer $\kappa \geq n$, the variety $\mathfrak{X} \subset \mathbb{P}\left(\mathbf{E}^{+}\right)$defines 'rank-one loci of incident planes',

$$
\tilde{\mathbb{I}}_{\kappa}(\mathfrak{X}):=\pi_{1}^{-1}(\mathfrak{X}) \subset \mathbb{F}_{\kappa}\left(\mathbf{E}^{+}\right) \text {and } \mathbb{I}_{\kappa}(\mathfrak{X}):=\pi_{2}\left(\pi_{1}^{-1}(\mathfrak{X})\right) \subset \mathbb{G}_{\kappa}\left(\mathbf{E}^{+}\right) .
$$


Corollary 4.8 implies that we shall only need to consider the incident loci $\tilde{\mathbb{I}}_{\kappa}\left(\mathfrak{X}_{\mathrm{sm}}\right)$ and $\mathbb{I}_{\kappa}\left(\mathfrak{X}_{\mathrm{sm}}\right)$ of the smooth part of $\mathfrak{X}$. In this case, $\tilde{\mathbb{I}}_{\kappa}\left(\mathfrak{X}_{\mathrm{sm}}\right)$ is a smooth submanifold of $\mathbb{F}_{\kappa}\left(\mathbf{E}^{+}\right)$with codimension

$$
\operatorname{codim}\left(\tilde{\mathbb{I}}_{\kappa}\left(\mathfrak{X}_{\mathrm{sm}}\right) ; \mathbb{F}_{\kappa}\left(\mathbf{E}^{+}\right)\right)=\operatorname{codim}\left(\mathfrak{X}_{\mathrm{sm}} ; \mathbb{P}\left(\mathbf{E}^{+}\right)\right)=\infty
$$

although $\mathbb{I}_{\kappa}\left(\mathfrak{X}_{\mathrm{sm}}\right)$ is not necessarily a smooth submanifold of $\mathbb{G}_{\kappa}\left(\mathbf{E}^{+}\right)$.

As we saw in $\S 4.2$, the Grassmann manifolds $\mathbb{G}_{\kappa}\left(\mathbf{E}^{+}\right)$or $\mathbb{G}\left(\mathbf{E}^{+}\right)$do not provide suitable target manifolds for our Dirac-operator period map, so we instead consider the preimages of the rank-one loci in $\operatorname{Fred}_{\kappa, n}\left(\mathbf{E}^{+}, \mathbf{E}^{-}\right)$using the projection (4.5) and then, in turn, in $\operatorname{Flag}_{\kappa, n}\left(\mathbf{E}^{+}, \mathbf{E}^{-}\right)$. Define

$$
\mathbb{J}_{\kappa}(\mathfrak{X}):=\pi^{-1}\left(\mathbb{I}_{\kappa}(\mathfrak{X})\right) \subset \operatorname{Fred}_{\kappa, n}\left(\mathbf{E}^{+}, \mathbf{E}^{-}\right) \subset \operatorname{Fred}_{n}\left(\mathbf{E}^{+}, \mathbf{E}^{-}\right),
$$

where $\pi:$ Fred $_{\kappa, n}\left(\mathbf{E}^{+}, \mathbf{E}^{-}\right) \rightarrow \mathbb{G}_{\kappa}\left(\mathbf{E}^{+}\right)$is the canonical map. The space $\mathbb{J}_{\kappa}(\mathfrak{X})$ need not be a smooth manifold, so we also define

$$
\tilde{\mathbb{J}}_{\kappa}(\mathfrak{X}):=\pi^{-1}\left(\tilde{\mathbb{I}}_{\kappa}(\mathfrak{X})\right) \subset \operatorname{Flag}_{\kappa, n}\left(\mathbf{E}^{+}, \mathbf{E}^{-}\right),
$$

which is a smooth submanifold since the canonical map

$$
\pi: \operatorname{Flag}_{\kappa, n}\left(\mathbf{E}^{+}, \mathbf{E}^{-}\right) \rightarrow \mathbb{F}_{\kappa}\left(\mathbf{E}^{+}\right)
$$

is a submersion according to Lemma 4.6. This last observation also implies

$$
\operatorname{codim}\left(\tilde{\mathbb{I}}_{\kappa}\left(\mathfrak{X}_{\mathrm{sm}}\right) ; \operatorname{Flag}_{\kappa, n}\left(\mathbf{E}^{+}, \mathbf{E}^{-}\right)\right)=\operatorname{codim}\left(\tilde{\mathbb{I}}_{\kappa}\left(\mathfrak{X}_{\mathrm{sm}}\right) ; \mathbb{F}_{\kappa}\left(\mathbf{E}^{+}\right)\right)=\infty
$$

courtesy of (4.8).

\subsection{The Dirac-operator period map and its differential.}

Recall that the principal goal of $\S 4$ is to show that for generic parameters $(f, \vartheta)$ and any point $[A, \Phi]$ in $M_{W, E}^{*, 0}(f, \tau, \vartheta)$, the section $\Phi$ in $\operatorname{Ker} D_{A, f, \vartheta}$ is not rank one. Hence, it is natural to define a 'period map', using the Dirac operator, to detect the rank-one loci that we wish to avoid, namely $\mathbb{J}_{\kappa}(\mathfrak{X})$ in $\operatorname{Fred}_{\kappa, n}\left(\mathbf{E}^{+}, \mathbf{E}^{-}\right)$or $\tilde{\mathbb{J}}_{\kappa}(\mathfrak{X})$ in $\operatorname{Flag}_{\kappa, n}\left(\mathbf{E}^{+}, \mathbf{E}^{-}\right)$.

We therefore define a smooth, ${ }^{\circ} \mathcal{G}_{E}$-equivariant map

$$
\underline{P}: \tilde{\mathcal{C}}_{W, E} \times \mathcal{P} \rightarrow \operatorname{Fred}_{n}\left(\mathbf{E}^{+}, \mathbf{E}^{-}\right), \quad(A, \Phi, f, \vartheta) \mapsto \mathcal{D}_{A, f, \vartheta}
$$

by analogy with $[11, \S \mathrm{VI}],[15, \mathrm{Eq}$. (4.3.14)]. The map $\underline{P}$ could obviously be defined simply on $\mathcal{A}_{E} \times \mathcal{P}$, but we shall soon need to consider it as a 
map on the universal moduli space $\tilde{\mathfrak{M}}_{W, E}^{*, 0} \subset \tilde{\mathcal{C}}_{W, E}^{*, 0} \times \mathcal{P}$. The map (4.12) has differential

$$
(D \underline{P})_{A, \Phi, f, \vartheta}: T_{A, \Phi} \tilde{\mathcal{C}}_{W, E} \oplus T_{f, \vartheta} \mathcal{P} \rightarrow \operatorname{Hom}_{\mathbb{C}}\left(\mathbf{E}^{+}, \mathbf{E}^{-}\right),
$$

given by Lemma 3.2,

$$
(a, \phi, \delta f, \delta \vartheta) \mapsto(D \mathcal{D})_{A, f, \vartheta}(a, \delta f, \delta \vartheta),
$$

noting that $T_{\underline{P}(A, \Phi, f, \vartheta)} \operatorname{Fred}_{n}\left(\mathbf{E}^{+}, \mathbf{E}^{-}\right)=\operatorname{Hom}_{\mathbb{C}}\left(\mathbf{E}^{+}, \mathbf{E}^{-}\right)$. We then have the following analogue of Proposition 4.3.14 in [15].

Proposition 4.9. Suppose $(A, \Phi, f, \vartheta)$ represents a point in the universal moduli space $\mathfrak{M}_{W, E}^{*, 0} \subset \mathcal{C}_{W, E}^{*, 0} \times \mathcal{P}$. Then the following partial differential is surjective:

$$
(D \underline{P})_{A, \Phi, f, \vartheta}(0, \cdot):\{0\} \oplus T_{f, \vartheta} \mathcal{P} \rightarrow T_{\underline{P}(A, \Phi, f, \vartheta)} \operatorname{Fred}_{n}\left(\mathbf{E}^{+}, \mathbf{E}^{-}\right),
$$

where $(D \underline{P}(A \Phi, \cdot))_{f, \vartheta}=(D \underline{P})_{A, \Phi, f, \vartheta}(0, \cdot)$.

Proposition 4.9 plays a crucial role in the proof of Theorem 4.1. As we shall see below, it follows easily from

Proposition 4.10. Assume $(A, \Phi)$ is an irreducible, non-zero-section $\mathrm{PU}(2)$ monopole on $X$ for the perturbation parameters $f, \vartheta$ (and some $\tau$ ). If $\phi \in \Omega^{0}\left(W^{+} \otimes E\right)$ and $\psi \in \Omega^{0}\left(W^{-} \otimes E\right)$ satisfy

$$
\operatorname{Re}\left(\left(D \mathcal{D}_{A}\right)_{f, \vartheta}(\delta f, \delta \vartheta), \psi \otimes \phi^{*}\right)_{L^{2}(X)}=0
$$

for all $(\delta f, \delta \vartheta)$, then $\psi \otimes \phi^{*} \equiv 0$ on $X$.

It is important to remember that

$$
\operatorname{Ran}(D \underline{P})_{A, \Phi, f, \vartheta} \subset \operatorname{Hom}_{\mathbb{C}}\left(\mathbf{E}^{+}, \mathbf{E}^{-}\right)
$$

is only a real subspace and so if there are elements of the latter tangent space which do not lie in $\operatorname{Ran}(D \underline{P})_{A, \Phi, f, \vartheta}$, then we can only assume there are tangent vectors obeying the real $L^{2}$-orthogonality condition of Proposition 4.10. Our proof of Proposition 4.10 ultimately relies on the following crucial 'unique continuation' result for reducible PU(2) monopoles:

Theorem 4.11 ([16, Theorem 5.11]). Suppose $(A, \Phi)$ is a solution to the perturbed $\mathrm{PU}(2)$ monopole equations (1.1) over a connected, oriented, smooth four-manifold $X$ with smooth Riemannian metric $g$ such that $(A, \Phi)$ is reducible on a non-empty open subset $U \subset X$. Then $(A, \Phi)$ is reducible on $X$ if 
- $\Phi \not \equiv 0$ on $X$, or

- $\Phi \equiv 0$, and $M_{E}^{\text {asd }}(g)$ contains no twisted reducibles or $U$ is suitable.

See [38, p.586] for the definition of a 'twisted reducible' and [38, p.589] for the definition of a 'suitable' open subset. A detailed proof of Theorem 4.11 is given in [16]. Our argument relies on the Agmon-Nirenberg unique continuation theorem for an ordinary differential equation on a Hilbert space [2], [3] and it generalizes the method used by Donaldson and Kronheimer to prove the corresponding unique continuation result for reducible antiself-dual connections [15, Lemma 4.3.21]. The proof of transversality via holonomy perturbations given in [16] relies on a refined version of this unique continuation property.

Proof of Proposition 4.10. Suppose $\psi \otimes \phi^{*} \not \equiv 0$ on $X$. According to Lemma 2.4 , varying $\delta \vartheta$ alone implies that both $\phi$ and $\psi$ have pointwise complexorthogonal images in $E$ and so they have at most complex rank one at each point of the subset $U:=\{\phi \neq 0\} \cap\{\psi \neq 0\} \subset X$, which we assume is non-empty. Over the open subset $U \subset X$, the section $\phi$ defines a Hermitian line subbundle $L_{1}:=\left.\left.\mathbb{C} \cdot \phi\right|_{U} \subset E\right|_{U}$. Let $\left.L_{2} \subset E\right|_{U}$ be the Hermitian line subbundle given by $L_{2}:=L_{1}^{\perp} \simeq\left(\left.E\right|_{U}\right) / L_{1}$, so $L_{2} \simeq\left(\left.\operatorname{det} E\right|_{U}\right) \otimes L_{1}^{*}$ and we have a unitary splitting of Hermitian vector bundles: $\left.E\right|_{U}=L_{1} \oplus L_{2}$. Furthermore, noting that $\phi, \psi$ have pointwise complex-orthogonal images in $E$, we have $\operatorname{Ran} \psi \subset L_{2}$.

With respect to this splitting, the unitary connection $\left.A\right|_{U}$ on $\left.E\right|_{U}$ may be written as

$$
\nabla_{A}=\left(\begin{array}{cc}
\nabla_{A_{1}} & -b^{*} \\
b & \nabla_{A_{2}}
\end{array}\right): \Omega^{0}\left(U, L_{1} \oplus L_{2}\right) \rightarrow \Omega^{1}\left(U, L_{1} \oplus L_{2}\right),
$$

where $A_{i}$ is a unitary connection on $L_{i}, i=1,2$, and $b \in \Omega^{1}\left(U, L_{2} \otimes L_{1}^{*}\right)$ is the second fundamental form of the pair $\left(A_{1}, L_{1}\right)$ with respect to $\left.(A, E)\right|_{U}$, and $b^{*}$ is the conjugate transpose of $b[33, \S I .6]$. (In terms of holomorphic bundles over a complex surface $X$, the second fundamental form $b$ may be identified with an element of $\operatorname{Ext}^{1}\left(L_{1}, L_{2}\right)$.) Clearly, $\left.A\right|_{U}$ is a reducible connection with respect to the splitting $\left.E\right|_{U}=L_{1} \oplus L_{2}$ if and only if $b \equiv 0$. Since $(A, \Phi)$ is irreducible and non-zero-section by hypothesis, Theorem 4.11 implies that $\left.(A, \Phi)\right|_{U}$ cannot be reducible and so $b \not \equiv 0$ on the non-empty open subset $U \subset X$.

From Lemma 3.2 and the splitting $\left.A\right|_{U}=A_{1} \oplus A_{2}$ on $\left.E\right|_{U}=L_{1} \oplus L_{2}$, 
we have (over $U \subset X$ )

$\nabla_{A} \phi=\left(\begin{array}{cc}\nabla_{A_{1}} & -b^{*} \\ b & \nabla_{A_{2}}\end{array}\right)\left(\begin{array}{c}\phi \\ 0\end{array}\right)=\left(\begin{array}{c}\nabla_{A_{1}} \phi \\ b \phi\end{array}\right) \in \Omega^{1}\left(U, W^{+} \otimes L_{1}\right) \oplus \Omega^{1}\left(U, W^{+} \otimes L_{2}\right)$.

Therefore,

$$
\begin{aligned}
\left(D \mathcal{D}_{A}\right)_{f, \vartheta}(\delta f, \delta \vartheta) \phi & =\sum_{i=1}^{4} \rho_{+}\left((\delta f) e^{i}\right) \nabla_{A, e_{i}} \phi+\rho_{+}(f(\delta \vartheta)) \phi \\
& =\sum_{i=1}^{4} \rho_{+}\left((\delta f) e^{i}\right) \nabla_{A_{1}, e_{i}} \phi \\
& +\sum_{i=1}^{4} \rho_{+}\left((\delta f) e^{i}\right) b\left(e_{i}\right) \phi+\rho_{+}(f(\delta \vartheta)) \phi
\end{aligned}
$$

where $\left\{e_{i}\right\}$ is a local oriented, orthonormal frame for $T X$, with dual coframe $\left\{e^{i}\right\}$ for $T^{*} X$. Thus,

$$
\begin{aligned}
\left(D \mathcal{D}_{A}\right)_{f, \vartheta}(\delta f, \delta \vartheta) \phi & =\sum_{i=1}^{4} \rho_{+}\left((\delta f) e^{i}\right) \nabla_{A_{1}, e_{i}} \phi \\
& +\rho_{+}(f(\delta \vartheta)) \phi+\rho_{+}((\delta f) b) \phi
\end{aligned}
$$

The first two terms on the right-hand side of (4.4) are in $\Omega^{0}\left(U, W^{-} \otimes L_{1}\right)$, while the last term is in $\Omega^{0}\left(U, W^{-} \otimes L_{2}\right)$. From (4.4), the fact that the sections $\phi, \psi$ have complex-orthogonal images in $E$ at each point of $X$, and the real $L^{2}$-orthogonality condition (4.13), we obtain

$$
\operatorname{Re}\left(\left(D \mathcal{D}_{A}\right)_{f, \vartheta}(\delta f, \delta \vartheta) \phi, \psi\right)_{L^{2}(X)}=\operatorname{Re}\left(\rho_{+}((\delta f) b) \phi, \psi\right)_{L^{2}(X)}=0
$$

for all $\delta f \in C^{\infty}\left(\mathfrak{g l}\left(T^{*} X\right)\right)$. Since $\phi$, $i \psi$ also have pointwise complexorthogonal images in $E$, we may replace $\psi$ by $i \psi$ above; combining the resulting two real $L^{2}$-orthogonality equations yields the complex $L^{2}$-orthogonality identity,

$$
\left(\rho_{+}((\delta f) b) \phi, \psi\right)_{L^{2}(X)}=0,
$$

for all $\delta f \in C^{\infty}\left(\mathfrak{g l}\left(T^{*} X\right)\right)$, which in turn, therefore, yields the pointwise identity:

$$
\left\langle\rho_{+}\left((\delta f)_{x} b_{x}\right) \phi_{x}, \psi_{x}\right\rangle_{x}=0, \quad x \in U
$$


for all $(\delta f)_{x} \in \mathfrak{g l}\left(T^{*} X\right)_{x}$. Note that

$$
\begin{aligned}
\rho_{+}((\delta f) b) & \in \operatorname{Hom}_{\mathbb{C}}\left(W^{+} \otimes_{\mathbb{C}} L_{1}, W^{-} \otimes_{\mathbb{C}} L_{2}\right) \\
& \simeq \operatorname{Hom}_{\mathbb{C}}\left(W^{+}, W^{-}\right) \otimes_{\mathbb{C}} \operatorname{Hom}_{\mathbb{C}}\left(L_{1}, L_{2}\right)
\end{aligned}
$$

and so

$$
\rho_{+}\left((\delta f)_{x} b_{x}\right) \in \operatorname{Hom}_{\mathbb{C}}\left(W^{+}, W^{-}\right)_{x} \otimes_{\mathbb{C}} \operatorname{Hom}_{\mathbb{C}}\left(L_{1}, L_{2}\right)_{x} \simeq \mathfrak{g l}(2, \mathbb{C}) .
$$

Since $\rho_{+}\left((\delta f)_{x} b_{x}\right) \phi_{x}$ and $\psi_{x}$ are orthogonal with respect to the full Hermitian inner product by (4.15), we may use the fact that the complexification $\rho_{+}\left(T^{*} X\right)_{x} \otimes_{\mathbb{R}} \mathbb{C}$ is equal to $\operatorname{Hom}_{\mathbb{C}}\left(W^{+}, W^{-}\right)_{x} \simeq \mathfrak{g l}(2, \mathbb{C})[48$, p. 89]. (See the proof in Lemma A.4.) Therefore, the identity (4.15) would yield

$$
\psi_{x} \otimes \phi_{x}^{*}=0 \in \operatorname{Hom}_{\mathbb{C}}\left(W^{+}, W^{-}\right)_{x},
$$

for all points $x \in U$ at which $b_{x} \neq 0$. Since $\psi_{x} \otimes \phi_{x}^{*} \neq 0$ for any point $x \in U$ by assumption, this would imply that $b \equiv 0$ on $U$ and so $\left.A\right|_{U}$ would be reducible, a contradiction. Hence, the open set $U \subset X$ must be empty and thus $\psi \otimes \phi^{*} \equiv 0$ on $X$, as desired.

We can now conclude the proof of Proposition 4.9:

Proof of Proposition 4.9. Suppose $(A, \Phi, f, \vartheta)$ represents a point in the universal moduli space $\mathfrak{M}_{W, E}^{*, 0}$. For convenience, we denote $p:=(f, \vartheta)$ and $\delta p:=(\delta f, \delta \vartheta)$. If $\left\{\phi_{a}\right\}_{a \in \mathbb{N}}$ is an orthonormal basis for the Hilbert space $\mathbf{E}^{+}$ and $\left\{\varphi_{b}\right\}_{b \in \mathbb{N}}$ is an orthonormal basis for the Hilbert space $\mathbf{E}^{-}$, then $\left\{\varphi_{b} \otimes\right.$ $\left.\phi_{a}^{*}\right\}_{(a, b) \in \mathbb{N} \times \mathbb{N}}$ is an orthonormal basis for the Hilbert space $\operatorname{Hom}_{\mathbb{C}}\left(\mathbf{E}^{+}, \mathbf{E}^{-}\right)$, where $\phi_{a}^{*}=\left\langle\cdot, \phi_{a}\right\rangle$. If $\operatorname{Ran}(D \underline{P})_{A, \Phi, p}(0, \cdot)=\operatorname{Ran}(D \underline{P}(A, \Phi, \cdot))_{p} \varsubsetneqq$ $T_{\underline{P}(A, \Phi, p)} \operatorname{Fred}_{n}\left(\mathbf{E}^{+}, \mathbf{E}^{-}\right)$, then there are sections $\phi \in \mathbf{E}^{+}$and $\varphi \in \mathbf{E}^{-}$, so

$$
\varphi \otimes \phi^{*} \in \operatorname{Hom}_{\mathbb{C}}\left(\mathbf{E}^{+}, \mathbf{E}^{-}\right)=T_{\mathcal{D}_{A, p}} \operatorname{Fred}_{n}\left(\mathbf{E}^{+}, \mathbf{E}^{-}\right),
$$

with $\varphi \otimes \phi^{*} \not \equiv 0$ on $X$ such that

$$
\operatorname{Re}\left((D \underline{P}(A, \Phi, \cdot))_{p}(\delta p), \varphi \otimes \phi^{*}\right)_{L^{2}(X)}=0,
$$

for all $\delta p$. Hence, for all $\delta p$ we have

$$
\operatorname{Re}\left(\left(D \mathcal{D}_{A}\right)_{p}(\delta p) \phi, \varphi\right)_{L^{2}(X)}=0
$$

and then Proposition 4.10 implies that $\varphi \otimes \phi^{*}=0$, a contradiction. Hence, the differential $(D \underline{P}(A, \Phi, \cdot))_{p}$ is surjective, as claimed. 


\subsection{The Sard-Smale theorem and transversality.}

We shall need a special form of the Sard-Smale theorem [65] for our proof of Theorem 4.1. The result is well-known and is essentially Proposition 4.3.10 in [15], but we shall require a more detailed statement than that given there, so we summarize the relevant discussion from $[15, \S 4.3 .1 \& \S 4.3 .2]$ and prove the precise version we need here.

Let $\mathcal{C}, \mathcal{P}$, and $\mathcal{F}$ be $C^{\infty}$ Banach manifolds. Suppose $\mathcal{M} \subset \mathcal{C} \times \mathcal{P}$ is a $C^{\infty}$ Banach submanifold and that the restriction $\pi_{\mathcal{M} ; \mathcal{P}}: \mathcal{M} \rightarrow \mathcal{P}$ of the projection $\pi_{\mathcal{P}}: \mathcal{C} \times \mathcal{P} \rightarrow \mathcal{P}$ onto the second factor is Fredholm. Let

$$
\underline{P}: \mathcal{M} \subset \mathcal{C} \times \mathcal{P} \rightarrow \mathcal{F}
$$

be a $C^{\infty}$ map which is transverse to a $C^{\infty}$ Banach submanifold $\mathcal{J} \subset \mathcal{F}$.

Proposition 4.12. Continue the notation of the preceding paragraph. Then there is a first-category subset $\mathcal{P}_{\mathrm{fc}} \subset \mathcal{P}$ such that the following holds. For all $p$ in $\mathcal{P}-\mathcal{P}_{\mathrm{fc}}$, we have

- $M:=\pi_{\mathcal{M} ; \mathcal{P}}^{-1}(p)$ is a $C^{\infty}$ manifold of dimension $\operatorname{ind}\left(\pi_{\mathcal{M} ; \mathcal{P}}\right)_{p}<\infty$,

- $P:=\underline{P}(\cdot, p): M \rightarrow \mathcal{F}$ is transverse to the submanifold $\mathcal{J} \subset \mathcal{F}$,

- $Z:=P^{-1}(\mathcal{J}) \subset M$ is a $C^{\infty}$ submanifold of codimension $\operatorname{codim}(Z ; M)=\operatorname{codim}(\mathcal{J} ; \mathcal{F})$.

Remark 4.13. When $\mathcal{C}$ is finite dimensional and $\mathcal{J}$ has finite codimension in $\mathcal{F}$, the preceding result is proved in $[1, \S 3.6 \mathrm{~B}]$.

Proof. The proof is similar to those of Propositions 4.3.10 and 4.3.11 in [15] (see [15, p. 143]). The first item follows immediately from the Sard-Smale theorem [65], for some first-category subset $\mathcal{P}_{\mathrm{fc}} \subset \mathcal{P}$. The hypotheses imply that the preimage

$$
\mathcal{Z}:=\underline{P}^{-1}(\mathcal{J}) \subset \mathcal{M} \subset \mathcal{C} \times \mathcal{P}
$$

is a $C^{\infty}$ Banach submanifold of $\mathcal{M}$. Let $p$ be a regular value of the projections

$$
\begin{aligned}
& \pi_{\mathcal{Z} ; \mathcal{P}}: \mathcal{Z} \subset \mathcal{C} \times \mathcal{P} \rightarrow \mathcal{P} \\
& \pi_{\mathcal{M} ; \mathcal{P}}: \mathcal{M} \subset \mathcal{C} \times \mathcal{P} \rightarrow \mathcal{P}
\end{aligned}
$$


so $p \in \mathcal{P}-\mathcal{P}_{\text {fc }}$, for some possibly larger first-category subset $\mathcal{P}_{\mathrm{fc}} \subset \mathcal{P}$, and let $(c, p)$ be any point in the fiber

$$
Z:=\pi_{\mathcal{Z} ; \mathcal{P}}^{-1}(p)=\underline{P}(\cdot, p)^{-1}(\mathcal{J})=P^{-1}(\mathcal{J}),
$$

and let $h=\underline{P}(c, p) \in \mathcal{F}$. Similarly, let $M:=\pi_{\mathcal{M} ; \mathcal{P}}^{-1}(p)$ and note that $Z$ and $M$ are $C^{\infty}$ manifolds.

Note that $T_{(c, p)} \mathcal{Z}$ and $T_{(c, p)} \mathcal{M}$ are subspaces of $T_{c} \mathcal{C} \oplus T_{p} \mathcal{P}$. By choice of $p \in \mathcal{P}-\mathcal{P}_{\mathrm{fc}}$ and by hypothesis, respectively, the maps

$$
\begin{aligned}
\left(D \pi_{\mathcal{Z} ; \mathcal{P}}\right)_{(c, p)} & : T_{(c, p)} \mathcal{Z} \rightarrow T_{p} \mathcal{P} \\
(D \underline{P})_{(c, p)} & : T_{(c, p)} \mathcal{M} \rightarrow T_{h} \mathcal{F} \rightarrow T_{h} \mathcal{F} / T_{h} \mathcal{J}
\end{aligned}
$$

are surjective and

$$
\begin{aligned}
& T_{c} M \oplus\{0\}=\left(D \pi_{\mathcal{M} ; \mathcal{P}}\right)_{(c, p)}^{-1}(0) \subset T_{(c, p)} \mathcal{M}, \\
& T_{(c, p)} \mathcal{Z}=(D \underline{P})_{(c, p)}^{-1}\left(T_{h} \mathcal{J}\right) \subset T_{(c, p)} \mathcal{M},
\end{aligned}
$$

where $T_{c} M \simeq T_{c} M \oplus\{0\} \subset T_{c} \mathcal{C} \oplus\{0\}$. We claim that the map $P: M \rightarrow \mathcal{F}$ is transverse to $\mathcal{J} \subset \mathcal{F}$, so the preimage $Z=P^{-1}(\mathcal{J})$ is a $C^{\infty}$ manifold of codimension $\operatorname{codim}(Z ; M)=\operatorname{codim}(\mathcal{J} ; \mathcal{F})$.

Suppose $\delta h \in T_{h} \mathcal{F}$. By (4.17) there is a tangent vector $(\delta c, \delta p) \in T_{(c, p)} \mathcal{M}$ such that $(D \underline{P})_{(c, p)}(\delta c, \delta p)=\delta h\left(\bmod T_{h} \mathcal{J}\right)$. According to $(4.16)$, given $\delta p$, there is a tangent vector of the form $\left(\delta c^{\prime}, \delta p\right) \in T_{(c, p)} \mathcal{Z}$ and so

$$
\left(\delta c-\delta c^{\prime}, 0\right)=(\delta c, \delta p)-\left(\delta c^{\prime}, \delta p\right) \in T_{c} M \oplus\{0\} \subset T_{(c, p)} \mathcal{M} .
$$

Since $(D \underline{P})_{(c, p)}\left(\delta c^{\prime}, \delta p\right) \in T_{h} \mathcal{J}$ (because $\left.\underline{P}^{-1}(\mathcal{J})=\mathcal{Z}\right)$ and $P=\underline{P}(\cdot, p)$, this gives

$$
\begin{aligned}
(D P)_{c}\left(\delta c-\delta c^{\prime}\right) & =(D \underline{P})_{(c, p)}\left(\delta c-\delta c^{\prime}, 0\right) \\
& =(D \underline{P})_{(c, p)}(\delta c, \delta p)-(D \underline{P})_{(c, p)}\left(\delta c^{\prime}, \delta p\right) \\
& =\delta h \quad\left(\bmod T_{h} \mathcal{J}\right) .
\end{aligned}
$$

Hence, the composition of the differential and quotient map,

$$
(D P)_{p}: T_{c} M \rightarrow T_{h} \mathcal{F} \rightarrow T_{h} \mathcal{F} / T_{h} \mathcal{J},
$$

is surjective, as desired. 


\subsection{The Sard-Smale theorem and semi-Kuranishi models.}

We shall use Proposition 4.12 to prove Theorem 4.1. As the detailed argument is technical and lengthy, we shall first outline the basic idea (under some simplifying assumptions) and establish a few notational conventions that will be useful in this section.

The space $\tilde{\mathcal{C}}_{W, E}^{*, 0} \times \mathcal{P}$ is a principal ${ }^{\circ} \mathcal{G}_{E^{-}}$-bundle over the base manifold $\mathcal{C}_{W, E}^{*, 0} \times \mathcal{P}$. The parametrized period map $\underline{P}$ may then be viewed as a section of the associated Banach vector bundle

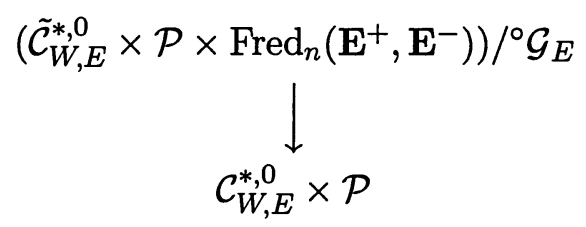

In order to obtain a Fredholm projection map onto the parameter space $\mathcal{P}$, we need to restrict the base of the preceding bundle to the universal moduli space $\mathfrak{M}_{W, E}^{*, 0} \subset \mathcal{C}_{W, E}^{*, 0} \times \mathcal{P}$. Of course, the space $\mathfrak{M}_{W, E}^{*, 0}$ is not necessarily a smooth manifold, so in the argument below we replace small open neighborhoods in $\mathfrak{M}_{W, E}^{*, 0}$ by 'thickened universal moduli spaces' containing the smooth locus of $\mathfrak{M}_{W, E}^{*, 0}$ as finite-dimensional submanifolds. Temporarily assuming $\mathfrak{M}_{W, E}^{*, 0}$ is smooth, for the purposes of this outline, we thus consider the Banach vector bundle together with a Fredholm projection map:

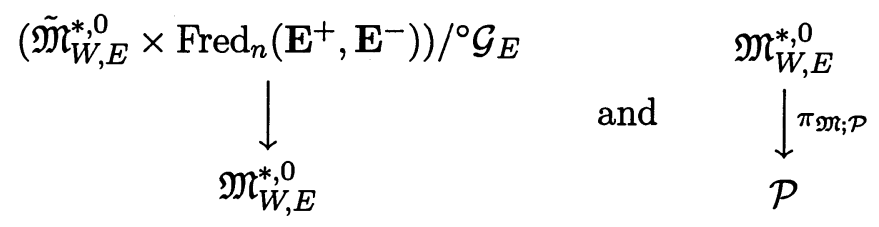

It is rather cumbersome to phrase the transversality argument in terms of sections of vector bundles rather than maps of Banach manifolds, although this can be done. So, before proceeding further, we restrict to slice neighborhoods in $\mathcal{C}_{W, E}^{*, 0}$ and $\mathfrak{M}_{W, E}^{*, 0}$, which for simplicity we continue to write as $\mathcal{C}_{W, E}^{*, 0}$ and $\mathfrak{M}_{W, E}^{*, 0}$ here, abusing notation slightly, rather than introduce new symbols. With respect to such a trivialization, we then have a parametrized period map and a Fredholm projection:

$$
\begin{aligned}
\underline{P}: \mathfrak{M}_{W, E}^{*, 0} \subset \mathcal{C}_{W, E}^{*, 0} \times \mathcal{P} & \rightarrow \operatorname{Fred}_{n}\left(\mathbf{E}^{+}, \mathbf{E}^{-}\right), \\
\pi_{\mathfrak{M} ; \mathcal{P}}: \mathfrak{M}_{W, E}^{*, 0} \subset \mathcal{C}_{W, E}^{*, 0} \times \mathcal{P} & \rightarrow \mathcal{P} .
\end{aligned}
$$


Fix an integer $\kappa \geq n:=$ ind $\mathcal{D}_{A, f, \vartheta}$ and temporarily assume, again for the purposes of this outline, that the rank-one locus $\mathbb{J}_{\kappa}\left(\mathfrak{X}_{\mathrm{sm}}\right) \subset \operatorname{Fred}_{n}\left(\mathbf{E}^{+}, \mathbf{E}^{-}\right)$ is a smooth submanifold. If the parametrized period map $\underline{P}: \mathfrak{M}_{W, E}^{*, 0} \rightarrow$ $\operatorname{Fred}_{n}\left(\mathbf{E}^{+}, \mathbf{E}^{-}\right)$is transverse to the locus $\mathbb{J}_{\kappa}\left(\mathfrak{X}_{\mathrm{sm}}\right) \subset \operatorname{Fred}_{n}\left(\mathbf{E}^{+}, \mathbf{E}^{-}\right)$, then Proposition 4.12 implies that the period map $P:=\underline{P}(\cdot, p)$ from the fiber $\pi_{\mathfrak{M} ; \mathcal{P}}^{-1}(p)=M_{W, E}^{*, 0}(p)$

$$
P: M_{W, E}^{*, 0}(p) \rightarrow \operatorname{Fred}_{n}\left(\mathbf{E}^{+}, \mathbf{E}^{-}\right),
$$

will be transverse to $\mathbb{J}_{\kappa}\left(\mathfrak{X}_{\mathrm{sm}}\right) \subset \operatorname{Fred}_{n}\left(\mathbf{E}^{+}, \mathbf{E}^{-}\right)$for all $p \in \mathcal{P}-\mathcal{P}_{\mathrm{fc}}$, for some first-category subset $\mathcal{P}_{\mathrm{fc}}$, and so

$$
\operatorname{codim}\left(P^{-1}\left(\mathbb{J}_{\kappa}\left(\mathfrak{X}_{\mathrm{sm}}\right) ; M_{W, E}^{*, 0}(p)\right)=\operatorname{codim}\left(\mathbb{J}_{\kappa}\left(\mathfrak{X}_{\mathrm{sm}}\right) ; \operatorname{Fred}_{n}\left(\mathbf{E}^{+}, \mathbf{E}^{-}\right)\right)=\infty .\right.
$$

Since $M_{W, E}^{*, 0}(p)$ is a finite-dimensional smooth manifold and $P^{-1}\left(\mathbb{J}_{\kappa}\left(\mathfrak{X}_{\mathrm{sm}}\right)\right)$ is a smooth submanifold with infinite codimension, the subset $P^{-1}\left(\mathbb{J}_{\kappa}\left(\mathfrak{X}_{\mathrm{sm}}\right)\right)$ is necessarily empty. Hence, after repeating this argument for each integer $\kappa \geq n$ and observing that the subset

$$
\begin{aligned}
& \bigcup_{\kappa \geq n} P^{-1}\left(\mathbb{J}_{\kappa}\left(\mathfrak{X}_{\mathrm{sm}}\right)\right) \\
& \quad=\left\{[A, \Phi] \in M_{W, E}^{*, 0}(p): \operatorname{Ker} D_{A, p} \text { contains some rank-one } \Psi\right\}
\end{aligned}
$$

is empty for generic $p \in \mathcal{P}$, we will have shown that for generic $p \in \mathcal{P}$, the moduli space $M_{W, E}^{*, 0}(p)$ contains no rank-one pairs.

Given this outline, we now proceed to the detailed proof of Theorem 4.1.

Proof of Theorem 4.1. Recall that in order to obtain a Fredholm projection map - thus permitting an application of the Sard-Smale theorem [15, $\S 4.3 .1 \&$ \&4.3.2], [65] in the form of Proposition 4.12 - we need to restrict our attention to the universal moduli space of irreducible, non-zero-section $\mathrm{PU}(2)$ monopoles:

$$
\mathfrak{M}_{W, E}^{*, 0}:=\left\{(A, \Phi, p) \in \tilde{\mathcal{C}}_{W, E}^{*, 0} \times \mathcal{P}: \mathfrak{S}(A, \Phi, p)=0\right\} /{ }^{\circ} \mathcal{G}_{E}
$$

Let $\left(A_{0}, \Phi_{0}, p_{0}\right)$ be any point in $\mathfrak{M}_{W, E}^{*, 0}$. The space $\mathfrak{M}_{W, E}^{*, 0}$ is not necessarily smooth so we first construct a semi-Kuranishi model for an open neighbor$\operatorname{hood}\left(A_{0}, \Phi_{0}, p_{0}\right)$ in $\mathfrak{M}_{W, E}^{*, 0}$. Let

$$
\mathbf{V}:=L_{k-1}^{2}\left(\Lambda^{+} \otimes \mathfrak{s u}(E)\right) \oplus L_{k-1}^{2}\left(W^{+} \otimes E\right)
$$


and observe that the map

$$
\underline{\mathfrak{S}}: \mathcal{C}_{W, E}^{*, 0} \times \mathcal{P} \rightarrow \mathbf{V}
$$

is right semi-Fredholm, in the sense that its differentials are right semiFredholm operators, having closed range and finite-dimensional cokernel (though not finite-dimensional kernels) [10, Definition XI.2.3 \& Proposition XI.2.10]:

Claim 4.14. The map $\underline{\mathfrak{S}}: \mathcal{C}_{W, E}^{*, 0} \times \mathcal{P} \rightarrow \mathbf{V}$ is right semi-Fredholm.

Proof. One can see this by observing that the differentials

$$
(D \underline{\mathfrak{S}})_{(A, \Phi, p)}: T_{(A, \Phi)} \mathcal{C}_{W, E}^{*, 0} \oplus T_{p} \mathcal{P} \rightarrow \mathbf{V}
$$

have right Green's operators defined by

$$
\begin{aligned}
& G_{(A, \Phi, p)} \\
& \quad=(D \underline{\mathfrak{S}})_{(A, \Phi, p)}^{\dagger}\left((D \underline{\mathfrak{S}})_{(A, \Phi, p)}(D \underline{\mathfrak{S}})_{(A, \Phi, p)}^{\dagger}\right)^{-1}: \mathbf{V} \rightarrow T_{(A, \Phi)} \mathcal{C}_{W, E}^{*, 0} \oplus T_{p} \mathcal{P} .
\end{aligned}
$$

Let $\Pi_{(A, \Phi, p)}$ denote the $L^{2}$-orthogonal projection from $\mathbf{V}$ onto the finitedimensional subspace

$$
\mathbf{H}_{(A, \Phi, p)}^{2}:=\left(\operatorname{Ran}(D \underline{S}(\cdot, p))_{(A, \Phi)}\right)^{\perp} \subset \mathbf{V},
$$

and let $\Pi_{(A, \Phi, p)}^{\perp}:=\mathrm{id}-\Pi_{(A, \Phi, p)}$ be the $L^{2}$-orthogonal projection from $\mathbf{V}$ onto the subspace $\operatorname{Ran}(D \underline{\mathfrak{S}}(\cdot, p))_{(A, \Phi)}$. Then,

$$
(D \underline{\mathfrak{S}})_{(A, \Phi, p)} \circ G_{(A, \Phi, p)}=\mathrm{id}-\Pi_{(A, \Phi, p)}: \mathbf{V} \rightarrow \mathbf{V}
$$

and so is right semi-Fredholm by [10, Definition XI.2.3], as $\Pi_{(A, \Phi, p)}$ is a finite-rank operator and thus compact.

We now consider the pair of maps

$$
\begin{aligned}
& \Pi_{\left(A_{0}, \Phi_{0}, p_{0}\right)}^{\perp} \circ \underline{\mathfrak{S}}: \mathcal{C}_{W, E}^{*, 0} \times \mathcal{P} \rightarrow\left(\mathbf{H}_{\left(A_{0}, \Phi_{0}, p_{0}\right)}^{2}\right)^{\perp} \cap \mathbf{V}, \\
& \Pi_{(A, 0},
\end{aligned}
$$

By construction, the differential of the stabilized canonical map (4.18) is surjective at the point $\left(A_{0}, \Phi_{0}, p_{0}\right)$ and thus also on some open neighborhood 
$\mathcal{U}_{\left(A_{0}, \Phi_{0}, p_{0}\right)}$ of the point $\left(A_{0}, \Phi_{0}, p_{0}\right)$ in $\mathcal{C}_{W, E}^{*, 0} \times \mathcal{P}$. An open neighborhood of $\left(A_{0}, \Phi_{0}, p_{0}\right)$ in $\mathfrak{M}_{W, E}^{*, 0}$ is then given by the zero locus of the finite-rank obstruction map (4.19) in the $C^{\infty}$ Banach manifold

$$
\mathcal{M}_{\left(A_{0}, \Phi_{0}, p_{0}\right)}:=\mathcal{U}_{\left(A_{0}, \Phi_{0}, p_{0}\right)} \cap\left(\Pi_{\left(A_{0}, \Phi_{0}, p_{0}\right)}^{\perp} \circ \underline{\mathfrak{S}}\right)^{-1}(0) \subset \mathcal{C}_{W, E}^{*, 0} \times \mathcal{P},
$$

comprising a thickened, parametrized moduli space. Let

$$
\pi_{\mathcal{M} ; \mathcal{P}}: \mathcal{M}_{\left(A_{0}, \Phi_{0}, p_{0}\right)} \rightarrow \mathcal{P}
$$

denote the restriction to $\mathcal{M}_{\left(A_{0}, \Phi_{0}, p_{0}\right)}$ of the projection from $\mathcal{C}_{W, E}^{*, 0} \times \mathcal{P}$ onto the second factor, and let

$$
\begin{aligned}
\mathbf{H}_{\left(A_{0}, \Phi_{0}, p_{0}\right)}^{1}: & =\operatorname{Ker}\left(D \underline{\mathfrak{S}}\left(\cdot, p_{0}\right)\right)_{\left(A_{0}, \Phi_{0}\right)} \\
& =\operatorname{Ker}\left(\Pi_{\left(A_{0}, \Phi_{0}, p_{0}\right)}^{\perp} \circ D \underline{\mathfrak{S}}\left(\cdot, p_{0}\right)\right)_{\left(A_{0}, \Phi_{0}\right)} \\
& =\operatorname{Ker}\left(D \pi_{\mathcal{M} ; \mathcal{P}}\right)_{\left(A_{0}, \Phi_{0}, p_{0}\right)} \subset T_{\left(A_{0}, \Phi_{0}\right)} \mathcal{C}_{W, E}^{*, 0}
\end{aligned}
$$

be the Zariski tangent space to the fiber $M_{W, E}^{*, 0}\left(p_{0}\right)$ at the point $\left(A_{0}, \Phi_{0}\right)$. Note that the differentials

$$
\left(D \pi_{\mathcal{M} ; \mathcal{P}}\right)_{(A, \Phi, p)}: T_{(A, \Phi, p)} \mathcal{M}_{\left(A_{0}, \Phi_{0}, p_{0}\right)} \rightarrow T_{p} \mathcal{P}
$$

are Fredholm and that $\mathbf{H}_{\left(A_{0}, \Phi_{0}, p_{0}\right)}^{1}$ is finite-dimensional.

We now consider the period map:

$$
\underline{P}: \mathcal{M}_{\left(A_{0}, \Phi_{0}, p_{0}\right)} \rightarrow \operatorname{Fred}_{n}\left(\mathbf{E}^{+}, \mathbf{E}^{-}\right) .
$$

The map $\underline{P}\left(A_{0}, \Phi_{0}, \cdot\right): \mathcal{P} \rightarrow \operatorname{Fred}_{n}\left(\mathbf{E}^{+}, \mathbf{E}^{-}\right)$is a submersion by Proposition 4.9 and in particular a submersion at the point $p_{0}$, but it does not follow that the same is true for the map (4.23) at the point $\left(A_{0}, \Phi_{0}, p_{0}\right)$ since the tangent space $T_{\left(A_{0}, \Phi_{0}, p_{0}\right)} \mathcal{M}_{\left(A_{0}, \Phi_{0}, p_{0}\right)}$ does not necessarily contain the subspace

$$
\{0\} \oplus T_{p_{0}} \mathcal{P} \subset T_{\left(A_{0}, \Phi_{0}\right)} \mathcal{C}_{W, E}^{*, 0} \oplus T_{p_{0}} \mathcal{P},
$$

as required by the hypotheses of Proposition 4.9. To circumvent this problem and permit an analysis of the preimage of $\mathbb{J}_{\kappa}\left(\mathfrak{X}_{\mathrm{sm}}\right)$ in $\mathcal{M}_{\left(A_{0}, \Phi_{0}, p_{0}\right)}$, we use a second stabilization technique similar to that employed by Donaldson for the anti-self-dual equation [11], [15, §7.2.2].

Claim 4.15. There is an equality of vector spaces,

$$
T_{\left(A_{0}, \Phi_{0}, p_{0}\right)} \mathcal{M}_{\left(A_{0}, \Phi_{0}, p_{0}\right)}+\left(\{0\} \oplus T_{p_{0}} \mathcal{P}\right)=\mathbf{H}_{\left(A_{0}, \Phi_{0}, p_{0}\right)}^{1} \oplus T_{p_{0}} \mathcal{P}
$$


and an isomorphism of vector spaces,

$$
\begin{aligned}
\left(\mathbf{H}_{\left(A_{0}, \Phi_{0}, p_{0}\right)}^{1} \oplus T_{p_{0}} \mathcal{P}\right) / T_{\left(A_{0}, \Phi_{0}, p_{0}\right)} \mathcal{M}_{\left(A_{0}, \Phi_{0}, p_{0}\right)} \\
\simeq \operatorname{Coker}\left(D \pi_{\mathcal{M} ; \mathcal{P}}\right)_{\left(A_{0}, \Phi_{0}, p_{0}\right)},
\end{aligned}
$$

and so $T_{\left(A_{0}, \Phi_{0}, p_{0}\right)} \mathcal{M}_{\left(A_{0}, \Phi_{0}, p_{0}\right)}$ has finite codimension in $\mathbf{H}_{\left(A_{0}, \Phi_{0}, p_{0}\right)}^{1} \oplus T_{p_{0}} \mathcal{P}$.

Proof. For every tangent vector

$$
\delta p \in \operatorname{Ran}\left(D \pi_{\mathcal{M} ; \mathcal{P}}\right)_{\left(A_{0}, \Phi_{0}, p_{0}\right)} \subset T_{p_{0}} \mathcal{P},
$$

there is a corresponding tangent vector $(a, \phi, \delta p)$ in $T_{\left(A_{0}, \Phi_{0}, p_{0}\right)} \mathcal{M}_{\left(A_{0}, \Phi_{0}, p_{0}\right)}$, so the equality (4.24) follows. Since the differential (4.22) is Fredholm, the cokernel

$$
\left(\operatorname{Ran}\left(D \pi_{\mathcal{M} ; \mathcal{P}}\right)_{\left(A_{0}, \Phi_{0}, p_{0}\right)}\right)^{\perp} \simeq \operatorname{Coker}\left(D \pi_{\mathcal{M} ; \mathcal{P}}\right)_{\left(A_{0}, \Phi_{0}, p_{0}\right)}
$$

is finite dimensional. Indeed, using the isomorphisms

$$
\begin{aligned}
& T_{p_{0}} \mathcal{P} \simeq \operatorname{Ran}\left(D \pi_{\mathcal{M} ; \mathcal{P}}\right)_{\left(A_{0}, \Phi_{0}, p_{0}\right)} \oplus \operatorname{Coker}\left(D \pi_{\mathcal{M} ; \mathcal{P}}\right)_{\left(A_{0}, \Phi_{0}, p_{0}\right)}, \\
& T_{\left(A_{0}, \Phi_{0}, p_{0}\right)} \mathcal{M}_{\left(A_{0}, \Phi_{0}, p_{0}\right)} \simeq\left(\operatorname{Ker}\left(D \pi_{\mathcal{M} ; \mathcal{P}}\right)_{\left(A_{0}, \Phi_{0}, p_{0}\right)}\right)^{\perp} \oplus \mathbf{H}_{\left(A_{0}, \Phi_{0}, p_{0}\right)}^{1}, \\
& \simeq \operatorname{Ran}\left(D \pi_{\mathcal{M} ; \mathcal{P}}\right)_{\left(A_{0}, \Phi_{0}, p_{0}\right)} \oplus \mathbf{H}_{\left(A_{0}, \Phi_{0}, p_{0}\right)}^{1},
\end{aligned}
$$

(the second isomorphism above being due to (4.22)), we obtain the isomorphism (4.25). The assertion on codimension follows from the fact that $\operatorname{dim} \operatorname{Coker}\left(D \pi_{\mathcal{M} ; \mathcal{P}}\right)_{\left(A_{0}, \Phi_{0}, p_{0}\right)}<\infty$, since the projection $\pi_{\mathcal{M} ; \mathcal{P}}$ is Fredholm.

Proposition 4.9 asserts that the differential

$$
(D \underline{P})_{\left(A_{0}, \Phi_{0}, p_{0}\right)}:\{0\} \oplus T_{p_{0}} \mathcal{P} \rightarrow T_{\underline{P}\left(A_{0}, \Phi_{0}, p_{0}\right)} \operatorname{Fred}_{n}\left(\mathbf{E}^{+}, \mathbf{E}^{-}\right)
$$

is surjective and so the same is true for

$$
(D \underline{P})_{\left(A_{0}, \Phi_{0}, p_{0}\right)}: \mathbf{H}_{\left(A_{0}, \Phi_{0}, p_{0}\right)}^{1} \oplus T_{p_{0}} \mathcal{P} \rightarrow T_{\underline{P}\left(A_{0}, \Phi_{0}, p_{0}\right)} \operatorname{Fred}_{n}\left(\mathbf{E}^{+}, \mathbf{E}^{-}\right) .
$$

Claim 4.15 and its proof imply that

$$
\begin{aligned}
& \mathbf{H}_{\left(A_{0}, \Phi_{0}, p_{0}\right)}^{1} \oplus T_{p_{0}} \mathcal{P} \\
& \quad \simeq \mathbf{H}_{\left(A_{0}, \Phi_{0}, p_{0}\right)}^{1} \oplus\left(\operatorname{Ker}\left(D \pi_{\mathcal{M} ; \mathcal{P}}\right)_{\left(A_{0}, \Phi_{0}, p_{0}\right)}\right)^{\perp} \oplus \operatorname{Coker}\left(D \pi_{\mathcal{M} ; \mathcal{P}}\right)_{\left(A_{0}, \Phi_{0}, p_{0}\right)} \\
& \quad \simeq T_{\left(A_{0}, \Phi_{0}, p_{0}\right)} \mathcal{M}_{\left(A_{0}, \Phi_{0}, p_{0}\right)} \oplus \operatorname{Coker}\left(D \pi_{\mathcal{M} ; \mathcal{P}}\right)_{\left(A_{0}, \Phi_{0}, p_{0}\right)} .
\end{aligned}
$$


Define a finite-dimensional subspace

$$
\begin{aligned}
F_{\left(A_{0}, \Phi_{0}, p_{0}\right)}: & =(D \underline{P})_{\left(A_{0}, \Phi_{0}, p_{0}\right)}\left(\operatorname{Coker}\left(D \pi_{\mathcal{M} ; \mathcal{P}}\right)_{\left(A_{0}, \Phi_{0}, p_{0}\right)}\right) \\
& \subset T_{\underline{P}\left(A_{0}, \Phi_{0}, p_{0}\right)} \operatorname{Fred}_{n}\left(\mathbf{E}^{+}, \mathbf{E}^{-}\right)
\end{aligned}
$$

and let

$$
\iota: F_{\left(A_{0}, \Phi_{0}, p_{0}\right)} \rightarrow T_{P\left(A_{0}, \Phi_{0}, p_{0}\right)} \operatorname{Fred}_{n}\left(\mathbf{E}^{+}, \mathbf{E}^{-}\right)=\operatorname{Hom}_{\mathbb{C}}\left(\mathbf{E}^{+}, \mathbf{E}^{-}\right)
$$

denote the inclusion. Although the differential

$$
(D \underline{P})_{\left(A_{0}, \Phi_{0}, p_{0}\right)}: T_{\left(A_{0}, \Phi_{0}, p_{0}\right)} \mathcal{M}_{\left(A_{0}, \Phi_{0}, p_{0}\right)} \rightarrow T_{\underline{P}\left(A_{0}, \Phi_{0}, p_{0}\right)} \operatorname{Fred}_{n}\left(\mathbf{E}^{+}, \mathbf{E}^{-}\right)
$$

need not necessarily be surjective, the following linear map is surjective by construction:

$$
\begin{aligned}
\iota+(D \underline{P})_{\left(A_{0}, \Phi_{0}, p_{0}\right)}: F_{\left(A_{0}, \Phi_{0}, p_{0}\right)} \oplus & T_{\left(A_{0}, \Phi_{0}, p_{0}\right)} \mathcal{M}_{\left(A_{0}, \Phi_{0}, p_{0}\right)} \\
& \rightarrow T_{\underline{P}\left(A_{0}, \Phi_{0}, p_{0}\right)} \operatorname{Fred}_{n}\left(\mathbf{E}^{+}, \mathbf{E}^{-}\right)
\end{aligned}
$$

where, for all $(\mathfrak{f}, \delta \mathfrak{m}) \in F_{\left(A_{0}, \Phi_{0}, p_{0}\right)} \oplus T_{\left(A_{0}, \Phi_{0}, p_{0}\right)} \mathcal{M}_{\left(A_{0}, \Phi_{0}, p_{0}\right)}$, we set

$$
\left(\iota+(D \underline{P})_{\left(A_{0}, \Phi_{0}, p_{0}\right)}\right)(\mathfrak{f}, \delta \mathfrak{m}):=\iota(\mathfrak{f})+(D \underline{P})_{\left(A_{0}, \Phi_{0}, p_{0}\right)}(\delta \mathfrak{m}) .
$$

Define a stabilized period map

$$
\iota+\underline{P}: F_{\left(A_{0}, \Phi_{0}, p_{0}\right)} \times \mathcal{M}_{\left(A_{0}, \Phi_{0}, p_{0}\right)} \rightarrow \operatorname{Fred}_{n}\left(\mathbf{E}^{+}, \mathbf{E}^{-}\right)
$$

by setting $(\iota+\underline{P})(\mathfrak{f}, \mathfrak{m}):=\iota(\mathfrak{f})+\underline{P}(\mathfrak{m})$, for all $(\mathfrak{f}, \mathfrak{m})$ in $F_{\left(A_{0}, \Phi_{0}, p_{0}\right)} \times \mathcal{M}_{\left(A_{0}, \Phi_{0}, p_{0}\right)}$. (We should write $\operatorname{Hom}_{\mathbb{C}}\left(\mathbf{E}^{+}, \mathbf{E}^{-}\right.$) for the image space in (4.27), but when $\mathfrak{f}$ is close enough to the origin in $F_{\left(A_{0}, \Phi_{0}, p_{0}\right)}$, the point $\iota(\mathfrak{f})+\underline{P}(\mathfrak{m})$ lies in the open subset $\operatorname{Fred}_{n}\left(\mathbf{E}^{+}, \mathbf{E}^{-}\right)$since $\underline{P}(\mathfrak{m})$ lies in $\operatorname{Fred}_{n}\left(\mathbf{E}^{+}, \mathbf{E}^{-}\right)$.) By construction, the differential (4.26) of the stabilized period map (4.27) is surjective at the point $\left(0, A_{0}, \Phi_{0}, p_{0}\right)$ and so there are an open neighborhood of the origin,

$$
O_{\left(A_{0}, \Phi_{0}, p_{0}\right)} \subset F_{\left(A_{0}, \Phi_{0}, p_{0}\right)},
$$

and, in the definition (4.20) of $\mathcal{M}_{\left(A_{0}, \Phi_{0}, p_{0}\right)}$, a possibly smaller open neighborhood $\mathcal{U}_{\left(A_{0}, \Phi_{0}, p_{0}\right)}$ of the point $\left(A_{0}, \Phi_{0}, p_{0}\right)$ in $\mathcal{C}_{W, E}^{*, 0} \times \mathcal{P}$, such that the restriction

$$
\iota+\underline{P}: O_{\left(A_{0}, \Phi_{0}, p_{0}\right)} \times \mathcal{M}_{\left(A_{0}, \Phi_{0}, p_{0}\right)} \rightarrow \operatorname{Fred}_{n}\left(\mathbf{E}^{+}, \mathbf{E}^{-}\right)
$$

of the map (4.27) is a submersion. 
Suppose $\kappa \geq n$ is an integer. Because the smooth map (4.28) is a submersion, the preimage

$$
\begin{aligned}
\mathcal{N}_{\left(A_{0}, \Phi_{0}, p_{0}\right)}^{\kappa}: & =\left(O_{\left(A_{0}, \Phi_{0}, p_{0}\right)} \times \mathcal{M}_{\left(A_{0}, \Phi_{0}, p_{0}\right)}\right) \\
& \cap(\iota+\underline{P})^{-1}\left(\operatorname{Fred}_{\kappa, n}\left(\mathbf{E}^{+}, \mathbf{E}^{-}\right)\right)
\end{aligned}
$$

is a smooth submanifold of $O_{\left(A_{0}, \Phi_{0}, p_{0}\right)} \times \mathcal{M}_{\left(A_{0}, \Phi_{0}, p_{0}\right)}$, with (finite) real codimension

$$
\operatorname{codim}_{\mathbb{R}}\left(\mathcal{N}_{\left(A_{0}, \Phi_{0}, p_{0}\right)}^{\kappa} ; O_{\left(A_{0}, \Phi_{0}, p_{0}\right)} \times \mathcal{M}_{\left(A_{0}, \Phi_{0}, p_{0}\right)}\right)=2 \kappa(\kappa-n),
$$

and so

$$
O_{\left(A_{0}, \Phi_{0}, p_{0}\right)} \times \mathcal{M}_{\left(A_{0}, \Phi_{0}, p_{0}\right)}=\bigcup_{\kappa \geq n} \mathcal{N}_{\left(A_{0}, \Phi_{0}, p_{0}\right)}^{\kappa}
$$

is a countable, disjoint union of smooth submanifolds.

Claim 4.16. The following smooth map is a submersion:

$$
\iota+\underline{P}: \mathcal{N}_{\left(A_{0}, \Phi_{0}, p_{0}\right)}^{\kappa} \rightarrow \operatorname{Fred}_{\kappa, n}\left(\mathbf{E}^{+}, \mathbf{E}^{-}\right) .
$$

Proof. Let $(A, \Phi, p)$ be a point in $\mathcal{N}_{\left(A_{0}, \Phi_{0}, p_{0}\right)}^{\kappa}$. From the definition (4.29) of the submanifold $\mathcal{N}_{\left(A_{0}, \Phi_{0}, p_{0}\right)}^{\kappa}$ and the fact that the map (4.28) is a submersion, we see that

$$
T_{(A, \Phi, p)} \mathcal{N}_{\left(A_{0}, \Phi_{0}, p_{0}\right)}^{\kappa}=(D(\iota+\underline{P}))_{(A, \Phi, p)}^{-1}\left(T_{(\iota+\underline{P})(A, \Phi, p)} \operatorname{Fred}_{\kappa, n}\left(\mathbf{E}^{+}, \mathbf{E}^{-}\right)\right),
$$

as the tangent space to the preimage is the preimage of the tangent space. Hence, it follows trivially that

$$
(D(\iota+\underline{P}))_{(A, \Phi, p)}\left(T_{(A, \Phi, p)} \mathcal{N}_{\left(A_{0}, \Phi_{0}, p_{0}\right)}^{\kappa}\right)=T_{(\iota+\underline{P})(A, \Phi, p)} \operatorname{Fred}_{\kappa, n}\left(\mathbf{E}^{+}, \mathbf{E}^{-}\right),
$$

which yields the claim.

While the manifold $\operatorname{Fred}_{\kappa, n}\left(\mathbf{E}^{+}, \mathbf{E}^{-}\right)$contains the rank-one locus $\mathbb{J}_{\kappa}\left(\mathfrak{X}_{\mathrm{sm}}\right)$, the latter space is not necessarily smooth and so it is convenient at this point to shift our attention instead to the smooth rank-one locus $\tilde{\mathbb{J}}_{\kappa}\left(\mathfrak{X}_{\mathrm{sm}}\right)$ in the flag manifold Flag ${ }_{\kappa, n}\left(\mathbf{E}^{+}, \mathbf{E}^{-}\right)$covering Fred $_{\kappa, n}\left(\mathbf{E}^{+}, \mathbf{E}^{-}\right)$. Recall that the canonical projection

$$
\operatorname{Flag}_{\kappa, n}\left(\mathbf{E}^{+}, \mathbf{E}^{-}\right) \rightarrow \operatorname{Fred}_{\kappa, n}\left(\mathbf{E}^{+}, \mathbf{E}^{-}\right)
$$


has finite-dimensional fibers $\mathbb{P}(\operatorname{Ker} B) \simeq \mathbb{P}^{\kappa-1}$ over points

$$
B \in \operatorname{Fred}_{\kappa, n}\left(\mathbf{E}^{+}, \mathbf{E}^{-}\right) .
$$

Then the submersion (4.31) then lifts to a smooth map

$$
\iota+\underline{\widetilde{P}}: \mathcal{N}_{\left(A_{0}, \Phi_{0}, p_{0}\right)}^{\kappa} \rightarrow \operatorname{Flag}_{\kappa, n}\left(\mathbf{E}^{+}, \mathbf{E}^{-}\right),
$$

though not necessarily to a submersion, given by

$$
(\mathfrak{f}, A, \Phi, p) \mapsto(\iota+\underline{\widetilde{P}})(\mathfrak{f}, A, \Phi, p),
$$

where

$$
(\iota+\underline{\widetilde{P}})(\mathfrak{f}, A, \Phi, p):=([\Phi], \iota(\mathfrak{f})+\underline{P}(A, \Phi, p))=\left([\Phi], \iota(\mathfrak{f})+\mathcal{D}_{A, p}\right) .
$$

The proof of the next lemma is the key application of Proposition 4.12.

Lemma 4.17. Continue the above notation. Then there is a first-category subset $\mathcal{P}_{\mathrm{fc}} \subset \mathcal{P}$, depending on $\left(A_{0}, \Phi_{0}, p_{0}\right)$, such that for all $p \in \mathcal{P}-\mathcal{P}_{\mathrm{fc}}$, the thickened moduli space $\left.\mathcal{M}_{\left(A_{0}, \Phi_{0}, p_{0}\right)}\right|_{p}:=\pi_{\mathcal{M} ; \mathcal{P}}^{-1}(p) \cap \mathcal{M}_{\left(A_{0}, \Phi_{0}, p_{0}\right)}$ contains no points $(A, \Phi, p)$ with $\Phi$ rank one.

Proof. From its definition, a countable union of first-category subsets of $\mathcal{P}$ is again a first-category subset, so it will suffice to consider a single open neighborhood of a point $\left(\mathfrak{f}_{1}, A_{1}, \Phi_{1}, p_{1}\right)$ in $\mathcal{N}_{\left(A_{0}, \Phi_{0}, p_{0}\right)}^{\kappa}$, as the space $\mathcal{N}_{\left(A_{0}, \Phi_{0}, p_{0}\right)}^{\kappa}$ is paracompact and so we may repeat the argument below for each element of a countable open cover.

We introduce a third stabilization, this time for the map (4.32), yielding a submersion onto $\operatorname{Flag}_{\kappa, n}\left(\mathbf{E}^{+}, \mathbf{E}^{-}\right)$. Let $\left\{\Phi_{1, \alpha}\right\}_{\alpha=1}^{\kappa}$ be an orthonormal basis for the kernel of $(\iota+\underline{P})\left(\mathfrak{f}_{1}, A_{1}, \Phi_{1}, p_{1}\right)=\iota\left(\mathfrak{f}_{1}\right)+\mathcal{D}_{A_{1}, p_{1}}$ and let

$$
\pi_{(\mathfrak{f}, A, \Phi, p)}: \mathbf{E}^{+} \rightarrow \operatorname{Ker}\left(\iota(\mathfrak{f})+\mathcal{D}_{A, p}\right)
$$

be the smooth family of $L^{2}$-orthogonal projections from $\mathbf{E}^{+}$onto $\operatorname{Ker}(\iota(\mathfrak{f})+$ $\left.\mathcal{D}_{A, p}\right)$, parametrized by the points $(\mathfrak{f}, A, \Phi, p)$ in $\mathcal{N}_{\left(A_{0}, \Phi_{0}, p_{0}\right)}^{\kappa}$. Let $\mathbf{z}:=$ $\left(z_{1}, \ldots, z_{\kappa}\right) \in \mathbb{C}^{\kappa}$ and define a smooth map

$$
\iota+\underline{\underline{P}}: \mathbb{C}^{\kappa} \times \mathcal{N}_{\left(A_{0}, \Phi_{0}, p_{0}\right)}^{\kappa} \rightarrow \operatorname{Flag}_{\kappa, n}\left(\mathbf{E}^{+}, \mathbf{E}^{-}\right)
$$

by the assignment $(\mathbf{z}, \mathfrak{f}, A, \Phi, p) \mapsto(\iota+\underline{\widehat{P}})(\mathbf{z}, \mathfrak{f}, A, \Phi, p)$, where

$$
\begin{aligned}
(\iota+\underline{\widehat{P}})(\mathbf{z}, \mathfrak{f}, A, \Phi, p) & :=\iota(\mathfrak{f})+\underline{\widehat{P}}(\mathbf{z}, A, \Phi, p) \\
& :=\left(\left[\Phi+\pi_{(\mathfrak{f}, A, \Phi, p)}\left(\sum_{\alpha=1}^{\kappa} z_{\alpha} \Phi_{1, \alpha}\right)\right], \iota(\mathfrak{f})+\mathcal{D}_{A, p}\right) .
\end{aligned}
$$


(Again, we should really use an open neighborhood of the origin in $\mathbb{C}^{\kappa}$ rather than all of $\mathbb{C}^{\kappa}$ in the definition of the map (4.34), so the vector in $\mathbf{E}^{+}$is non-zero and defines an element of $\mathbb{P}\left(\mathbf{E}^{+}\right)$; however, the map is clearly welldefined for $\mathbf{z}$ near zero and that is all we shall need below.) Note that when. $(\mathbf{z}, \mathfrak{f})=(0,0)$, the term $(\iota+\underline{\widehat{P}})(\mathbf{z}, \mathfrak{f}, A, \Phi, p)$ simplifies to

$$
\begin{aligned}
(\iota+\underline{\widehat{P}})(0,0, A, \Phi, p) & =\left([\Phi], \mathcal{D}_{A, p}\right) \\
& =\underline{\widetilde{P}}(A, \Phi, p) \\
& \in \mathbb{P}\left(\operatorname{Ker} \mathcal{D}_{A, p}\right) \times \operatorname{Fred}_{\kappa, n}\left(\mathbf{E}^{+}, \mathbf{E}^{-}\right),
\end{aligned}
$$

with $\mathbb{P}\left(\operatorname{Ker} \mathcal{D}_{A, p}\right) \subset \mathbb{P}\left(\mathbf{E}^{+}\right)$.

Claim 4.18. The map $(\iota+\underline{\underline{P}})$ of (4.34) is a submersion at the point $\left(0, \mathfrak{f}_{1}, A_{1}, \Phi_{1}, p_{1}\right)$.

Proof. From (4.34) we have $(\iota+\underline{\underline{\widehat{P}}})\left(0, \mathfrak{f}_{1}, A_{1}, \Phi_{1}, p_{1}\right)=\left(\left[\Phi_{1}\right], \iota\left(\mathfrak{f}_{1}\right)+\mathcal{D}_{A_{1}, p_{1}}\right)$. The map (4.31), given by $(\mathfrak{f}, A, \Phi, p) \mapsto \iota(\mathfrak{f})+\mathcal{D}_{A, p}$, is a submersion at $\left(\mathfrak{f}_{1}, A_{1}, \Phi_{1}, p_{1}\right)$. Hence, if $B_{t}$ is a smooth path in $\operatorname{Fred}_{\kappa, n}\left(\mathbf{E}^{+}, \mathbf{E}^{-}\right)$through $B_{1}:=\iota\left(\mathfrak{f}_{1}\right)+\mathcal{D}_{A_{1}, p_{1}}$, we may choose a smooth path $\left(\mathfrak{f}_{t}, A_{t}, \Phi_{t}, p_{t}\right)$ in $\mathcal{N}_{\left(A_{0}, \Phi_{0}, p_{0}\right)}^{\kappa}$, passing through $\left(\mathfrak{f}_{1}, A_{1}, \Phi_{1}, p_{1}\right)$, which maps to the smooth path $B_{t}$ near $t=1$ via (4.31), so

$$
B_{t}=\iota\left(\mathfrak{f}_{t}\right)+\mathcal{D}_{A_{t}, p_{t}} .
$$

Using (4.33), define a smooth path of projections onto $\operatorname{Ker} B_{t} \subset \mathbf{E}^{+}$by

$$
\pi_{t}:=\pi_{\left(\mathfrak{f}_{t}, A_{t}, \Phi_{t}, p_{t}\right)}, \quad t \text { near } 1 .
$$

Then, $\pi_{1}=$ id on $\operatorname{Ker} B_{1}$ and $\left\{\pi_{t}\left(\Phi_{1, \alpha}\right)\right\}_{\alpha=1}^{\kappa}$ is a basis for $\operatorname{Ker} B_{t}=$ $\operatorname{Ker}\left(\iota\left(\mathfrak{f}_{t}\right)+\mathcal{D}_{A_{t}, p_{t}}\right)$ near $t=1$, since $\left.\left\{\Phi_{1, \alpha}\right)\right\}_{\alpha=1}^{\kappa}$ was chosen to be a basis for $\operatorname{Ker} B_{1}$. Now suppose $\Psi_{t}$ is a smooth path in $\mathbf{E}^{+}$through $\Phi_{1}$ such that $B_{t} \Psi_{t}=0$, so $\left(\left[\Psi_{t}\right], B_{t}\right)$ is a smooth path in $\operatorname{Flag}_{\kappa, n}\left(\mathbf{E}^{+}, \mathbf{E}^{-}\right)$. Because $\Psi_{t}, \Phi_{t} \in \operatorname{Ker} B_{t}$, near $t=1$ we can write

$$
\Psi_{t}-\Phi_{t}=\sum_{\alpha=1}^{\kappa}\left(\Psi_{t}-\Phi_{t}, \pi_{t}\left(\Phi_{1, \alpha}\right)\right)_{L^{2}} \cdot \pi_{t}\left(\Phi_{1, \alpha}\right)=: \sum_{\alpha=1}^{\kappa} z_{\alpha}(t) \pi_{t}\left(\Phi_{1, \alpha}\right)
$$

with $z_{\alpha}(t)=0$ when $t=1$. Hence, near $t=1$, the path $\left(\left[\Psi_{t}\right], B_{t}\right)$ through $(\iota+\underline{\widehat{P}})\left(0, \mathfrak{f}_{1}, A_{1}, \Phi_{1}, p_{1}\right)$ in the image, Flag ${ }_{\kappa, n}\left(\mathbf{E}^{+}, \mathbf{E}^{-}\right)$, lifts to a smooth path 
$\left(\mathbf{z}_{t}, \mathfrak{f}_{t}, A_{t}, \Phi_{t}, p_{t}\right)$ in the domain, $\mathbb{C}^{\kappa} \times \mathcal{N}_{\left(A_{0}, \Phi_{0}, p_{0}\right)}^{\kappa}$, through $\left(0, \mathfrak{f}_{1}, A_{1}, \Phi_{1}, p_{1}\right)$ with

$$
(\iota+\underline{\widehat{P}})\left(\mathbf{z}_{t}, \mathfrak{f}_{t}, A_{t}, \Phi_{t}, p_{t}\right)=\left(\left[\Psi_{t}\right], B_{t}\right) .
$$

In particular, we have

$$
\left.\frac{d}{d t}(\iota+\underline{\underline{\widehat{P}}})\left(\mathbf{z}_{t}, \mathfrak{f}_{t}, A_{t}, \Phi_{t}, p_{t}\right)\right|_{t=1}=\left.\frac{d}{d t}\left(\left[\Psi_{t}\right], B_{t}\right)\right|_{t=1} .
$$

Since the smooth path $\left(\left[\Psi_{t}\right], B_{t}\right)$ through $(\iota+\underline{\widehat{P}})\left(0, \mathfrak{f}_{1}, A_{1}, \Phi_{1}, p_{1}\right)$ was arbitrary, we see that $\iota+\underline{\underline{P}}$ is a submersion at $\left(0, \mathfrak{f}_{1}, A_{1}, \Phi_{1}, p_{1}\right)$, as desired.

By Claim 4.18, the map (4.34) is a submersion at the point $\left(0, \mathfrak{f}_{1}, A_{1}, \Phi_{1}, p_{1}\right)$ and so is a submersion on an open neighborhood of this point in $\mathbb{C}^{\kappa} \times \mathcal{N}_{\left(A_{0}, \Phi_{0}, p_{0}\right)}^{\kappa}$. Rather than introduce further notation, we may suppose without loss of generality (see the remark at the beginning of the proof of the lemma concerning first-category sets) that the map (4.34) is a submersion on its entire domain $\mathbb{C}^{\kappa} \times \mathcal{N}_{\left(A_{0}, \Phi_{0}, p_{0}\right)}^{\kappa}$.

Consequently, the locus

$$
\mathcal{Z}_{\left(A_{0}, \Phi_{0}, p_{0}\right)}^{\kappa}:=\left(\mathbb{C}^{\kappa} \times \mathcal{N}_{\left(A_{0}, \Phi_{0}, p_{0}\right)}^{\kappa}\right) \cap(\iota+\underline{\widehat{P}})^{-1}\left(\tilde{\mathbb{J}}_{\kappa}\left(\mathfrak{X}_{\mathrm{sm}}\right)\right)
$$

is a $C^{\infty}$ Banach submanifold of $\mathbb{C}^{\kappa} \times \mathcal{N}_{\left(A_{0}, \Phi_{0}, p_{0}\right)}^{\kappa}$. Let

$$
\begin{aligned}
\mathcal{M}_{\left(A_{0}, \Phi_{0}, p_{0}\right)}^{\kappa}: & =\mathcal{N}_{\left(A_{0}, \Phi_{0}, p_{0}\right)}^{\kappa} \cap\left(\{0\} \times \mathcal{M}_{\left(A_{0}, \Phi_{0}, p_{0}\right)}\right) \\
& \subset O_{\left(A_{0}, \Phi_{0}, p_{0}\right)} \times \mathcal{M}_{\left(A_{0}, \Phi_{0}, p_{0}\right)},
\end{aligned}
$$

and observe that we have a countable disjoint union of subsets:

$$
\mathcal{M}_{\left(A_{0}, \Phi_{0}, p_{0}\right)}=\bigcup_{\kappa \geq n} \mathcal{M}_{\left(A_{0}, \Phi_{0}, p_{0}\right)}^{\kappa} .
$$

Of course, the unstabilized period map (4.23) also lifts to a map,

$$
\underline{\widetilde{P}}: \mathcal{M}_{\left(A_{0}, \Phi_{0}, p_{0}\right)}^{\kappa} \rightarrow \operatorname{Flag}_{\kappa, n}\left(\mathbf{E}^{+}, \mathbf{E}^{-}\right)
$$

defined, as in (4.32), by setting

$$
\underline{\widetilde{P}}(A, \Phi, p):=(\iota+\underline{\widetilde{P}})(0, A, \Phi, p)=\left([\Phi], \mathcal{D}_{A, p}\right)=([\Phi], \underline{P}(A, \Phi, p)) .
$$

(Note that the preimage $\underline{P}^{-1}\left(\mathbb{J}_{\kappa}\left(\mathfrak{X}_{\mathrm{sm}}\right)\right)$ in $\mathcal{M}_{\left(A_{0}, \Phi_{0}, p_{0}\right)}^{\kappa}$ consists of points $(A, \Phi, p)$ such that $\operatorname{Ker} \mathcal{D}_{A, p}$ contains some rank-one section, whereas the 
preimage $\underline{\widetilde{P}}^{-1}\left(\tilde{\mathbb{J}}_{\kappa}\left(\mathfrak{X}_{\mathrm{sm}}\right)\right)$ in $\mathcal{M}_{\left(A_{0}, \Phi_{0}, p_{0}\right)}^{\kappa}$ consists of points $(A, \Phi, p)$ with $\Phi$ a rank-one element of $\operatorname{Ker} \mathcal{D}_{A, p}$.)

Note that by (4.35) we have

$$
\begin{aligned}
& \mathcal{M}_{\left(A_{0}, \Phi_{0}, p_{0}\right)}^{\kappa} \cap \underline{\widetilde{P}}^{-1}\left(\tilde{\mathbb{J}}_{\kappa}\left(\mathfrak{X}_{\mathrm{sm}}\right)\right) \\
& =\left(\{0\} \times\{0\} \times \mathcal{M}_{\left(A_{0}, \Phi_{0}, p_{0}\right)}^{\kappa}\right) \cap(\iota+\underline{\widehat{P}})^{-1}\left(\tilde{\mathbb{J}}_{\kappa}\left(\mathfrak{X}_{\mathrm{sm}}\right)\right) \\
& =\left(\{0\} \times\{0\} \times \mathcal{M}_{\left(A_{0}, \Phi_{0}, p_{0}\right)}^{\kappa}\right) \cap \mathcal{Z}_{\left(A_{0}, \Phi_{0}, p_{0}\right)}^{\kappa} .
\end{aligned}
$$

Let $\pi_{C \times \mathcal{N} ; \mathcal{P}}$ be the projection to the factor $\mathcal{P}$ from the $C^{\infty}$ Banach submanifold

$$
\mathbb{C}^{\kappa} \times \mathcal{N}_{\left(A_{0}, \Phi_{0}, p_{0}\right)}^{\kappa} \subset \mathbb{C}^{\kappa} \times F_{\left(A_{0}, \Phi_{0}, p_{0}\right)} \times \mathcal{C}_{W, E}^{*, 0} \times \mathcal{P}
$$

and similarly define $\pi_{\mathcal{N} ; \mathcal{P}}: \mathcal{N}_{\left(A_{0}, \Phi_{0}, p_{0}\right)}^{\kappa} \rightarrow \mathcal{P}$. Proposition 4.12 now implies that there is a first-category subset $\mathcal{P}_{\mathrm{fc}} \subset \mathcal{P}$ such that for each $p \in \mathcal{P}-\mathcal{P}_{\mathrm{fc}}$, the preimages

$$
\mathbb{C}^{\kappa} \times\left.\mathcal{N}_{\left(A_{0}, \Phi_{0}, p_{0}\right)}^{\kappa}\right|_{p}:=\pi_{C \times \mathcal{N} ; \mathcal{P}}^{-1}(p) \cap\left(\mathbb{C}^{\kappa} \times \mathcal{N}_{\left(A_{0}, \Phi_{0}, p_{0}\right)}^{\kappa}\right),
$$

where $\left.\mathcal{N}_{\left(A_{0}, \Phi_{0}, p_{0}\right)}^{\kappa}\right|_{p}:=\pi_{\mathcal{N} ; \mathcal{P}}^{-1}(p) \cap \mathcal{N}_{\left(A_{0}, \Phi_{0}, p_{0}\right)}^{\kappa}$, and

$$
\left.\mathcal{Z}_{\left(A_{0}, \Phi_{0}, p_{0}\right)}^{\kappa}\right|_{p}:=(\iota+\underline{\widehat{P}}(\cdot, p))^{-1}\left(\tilde{\mathbb{J}}_{\kappa}\left(\mathfrak{X}_{\mathrm{sm}}\right)\right) \cap\left(\mathbb{C}^{\kappa} \times\left.\mathcal{N}_{\left(A_{0}, \Phi_{0}, p_{0}\right)}^{\kappa}\right|_{p}\right)
$$

are smooth submanifolds; the parameters $p \in \mathcal{P}-\mathcal{P}_{\mathrm{fc}}$ are regular values of the projections defining the above two preimages, which are $C^{\infty}$ and Fredholm.

Claim 4.19. The manifolds $\mathbb{C}^{\kappa} \times\left.\mathcal{N}_{\left(A_{0}, \Phi_{0}, p_{0}\right)}^{\kappa}\right|_{p}$ and $\left.\mathcal{Z}_{\left(A_{0}, \Phi_{0}, p_{0}\right)}^{\kappa}\right|_{p}$ have the following dimension and codimension, respectively:

$$
\begin{aligned}
& \left.\operatorname{dim}_{\mathbb{R}}\left(\mathbb{C}^{\kappa} \times \mathcal{N}_{\left(A_{0}, \Phi_{0}, p_{0}\right)}^{\kappa}\right)\right|_{p} \\
& \quad=\operatorname{dim}_{\mathbb{R}} F_{\left(A_{0}, \Phi_{0}, p_{0}\right)}+2 \kappa+\operatorname{dim} \mathbf{H}_{\left(A_{0}, \Phi_{0}, p_{0}\right)}^{1}-2 \kappa(\kappa-n)<\infty, \\
& \operatorname{codim}_{\mathbb{R}}\left(\left.\mathcal{Z}_{\left(A_{0}, \Phi_{0}, p_{0}\right)}^{\kappa}\right|_{p} ; \mathbb{C}^{\kappa} \times\left.\mathcal{N}_{\left(A_{0}, \Phi_{0}, p_{0}\right)}^{\kappa}\right|_{p}\right) \\
& \quad=\operatorname{codim}\left(\tilde{\mathbb{J}}_{\kappa}\left(\mathfrak{X}_{\mathrm{sm}}\right) ; \operatorname{Flag}_{\kappa, n}\left(\mathbf{E}^{+}, \mathbf{E}^{-}\right)\right)=\infty
\end{aligned}
$$

In particular, $\left.\mathcal{Z}_{\left(A_{0}, \Phi_{0}, p_{0}\right)}\right|_{p}$ is empty.

Proof. According to (4.29) and (4.38) we have

$$
\begin{aligned}
\mathbb{C}^{\kappa} & \times\left.\mathcal{N}_{\left(A_{0}, \Phi_{0}, p_{0}\right)}^{\kappa}\right|_{p} \\
& =\left(O_{\left(A_{0}, \Phi_{0}, p_{0}\right)} \times\left.\mathcal{M}_{\left(A_{0}, \Phi_{0}, p_{0}\right)}\right|_{p}\right) \cap(\iota+\underline{P}(\cdot, p))^{-1}\left(\operatorname{Fred}_{\kappa, n}\left(\mathbf{E}^{+}, \mathbf{E}^{-}\right)\right) .
\end{aligned}
$$


Hence, the expression for the dimension of $\mathbb{C}^{\kappa} \times\left.\mathcal{N}_{\left(A_{0}, \Phi_{0}, p_{0}\right)}^{\kappa}\right|_{p}$ follows from the facts that

$$
\operatorname{codim}_{\mathbb{R}}\left(\operatorname{Fred}_{\kappa, n}\left(\mathbf{E}^{+}, \mathbf{E}^{-}\right) ; \operatorname{Fred}_{n}\left(\mathbf{E}^{+}, \mathbf{E}^{-}\right)\right)=2 \kappa(\kappa-n),
$$

(just as in the derivation of (4.30)) in conjunction with Proposition 4.12 applied to the submersion (4.28) given by $\iota+\underline{P}$ on $O_{\left(A_{0}, \Phi_{0}, p_{0}\right)} \times \mathcal{M}_{\left(A_{0}, \Phi_{0}, p_{0}\right)}$ and the genericity of $p$, the fact that $\operatorname{dim}_{\mathbb{R}} F_{\left(A_{0}, \Phi_{0}, p_{0}\right)}=\operatorname{dim}_{\mathbb{R}} O_{\left(A_{0}, \Phi_{0}, p_{0}\right)}$, and the fiber dimension formula implied by (4.21), namely

$$
\left.\operatorname{dim} \mathcal{M}_{\left(A_{0}, \Phi_{0}, p_{0}\right)}\right|_{p}=\operatorname{dim} \mathbf{H}_{\left(A_{0}, \Phi_{0}, p_{0}\right)}^{1} .
$$

The equality in (4.41) follows from the fact that the map $\iota+\underline{\widehat{P}}(\cdot, p)$ is a submersion on $\mathbb{C}^{\kappa} \times\left.\mathcal{N}_{\left(A_{0}, \Phi_{0}, p_{0}\right)}^{\kappa}\right|_{p}$ according to Claim 4.18 and Proposition 4.12, for generic $p$. The infinite-codimension assertion for $\left.\mathcal{Z}_{\left(A_{0}, \Phi_{0}, p_{0}\right)}\right|_{p}$ follows from (4.11). Thus, $\left.\mathcal{Z}_{\left(A_{0}, \Phi_{0}, p_{0}\right)}\right|_{p}$ is a smooth manifold of negative dimension, by (4.40) and (4.41), and so is empty.

From (4.37), we see that (for any $p \in \mathcal{P}$ ) we have

$$
\left.\left.\mathcal{M}_{\left(A_{0}, \Phi_{0}, p_{0}\right)}^{\kappa}\right|_{p} \cap \underline{\widetilde{P}}(\cdot, p)^{-1}\left(\tilde{\mathbb{J}}_{\kappa}\left(\mathfrak{X}_{\mathrm{sm}}\right)\right) \subset \mathcal{Z}_{\left(A_{0}, \Phi_{0}, p_{0}\right)}^{\kappa}\right|_{p},
$$

and so, as $\left.\mathcal{Z}_{\left(A_{0}, \Phi_{0}, p_{0}\right)}\right|_{p}$ is empty according to Claim 4.19 , the intersection $\left.\mathcal{M}_{\left(A_{0}, \Phi_{0}, p_{0}\right)}^{\kappa}\right|_{p} \cap \underline{\widetilde{P}}(\cdot, p)^{-1}\left(\tilde{\mathbb{J}}_{\kappa}\left(\mathfrak{X}_{\mathrm{sm}}\right)\right)$ must be empty as well. Therefore, the thickened moduli space $\left.\mathcal{M}_{\left(A_{0}, \Phi_{0}, p_{0}\right)}\right|_{p}$ contains no points $(A, \Phi, p)$ with $\Phi$ rank one, when $\operatorname{dim}_{\mathbb{C}} \operatorname{Ker} \mathcal{D}_{A, p}=\kappa$ and $p \in \mathcal{P}_{\text {fc }}$. Repeating this argument for all $\kappa \geq n$ yields the desired conclusion, recalling that a countable union of first-category subsets of $\mathcal{P}$ is again a first-category subset. This completes the proof of Lemma 4.17 .

Since we have an inclusion

$$
\mathfrak{M}_{W, E}^{*, 0} \cap \mathcal{M}_{\left(A_{0}, \Phi_{0}, p_{0}\right)} \subset \mathcal{M}_{\left(A_{0}, \Phi_{0}, p_{0}\right)}
$$

of an open neighborhood of the point $\left(A_{0}, \Phi_{0}, p_{0}\right)$ in the parametrized moduli space $\mathfrak{M}_{W, E}^{*, 0}$ into the thickened, parametrized moduli space $\mathcal{M}_{\left(A_{0}, \Phi_{0}, p_{0}\right)}$, Lemma 4.17 implies that $\left.M_{W, E}^{*, 0}(p) \cap \mathcal{M}_{\left(A_{0}, \Phi_{0}, p_{0}\right)}\right|_{p}$ contains no points $(A, \Phi, p)$ with $\Phi$ rank one, when $p \in \mathcal{P}-\mathcal{P}_{\mathrm{fc}}$. We now repeat this process for every point $\left(A_{1}, \Phi_{1}, p_{1}\right)$ in $\mathfrak{M}_{W, E}^{*, 0}$, using appropriate open neighborhoods $\mathcal{U}_{\left(A_{1}, \Phi_{1}, p_{1}\right)}$ of $\left(A_{1}, \Phi_{1}, p_{1}\right)$ in $\mathcal{C}_{W, E}^{*, 0} \times \mathcal{P}$, and obtaining a first-category subset for each such neighborhood. Since $\mathcal{C}_{W, E}^{*, 0} \times \mathcal{P}$ is a paracompact Banach 
manifold, we may pass to a collection of open neighborhoods in $\mathcal{C}_{W, E}^{*, 0} \times \mathcal{P}$ which gives a countable open covering of $\mathfrak{M}_{W, E}^{*, 0}$. A countable union of firstcategory subsets of $\mathcal{P}$ is again a first-category subset, so this process yields the desired set $\mathcal{P}_{\mathrm{fc}} \subset \mathcal{P}$ in the case of $C^{r}$ parameters. That is, for each $C^{r}$ parameter $p \in \mathcal{P}-\mathcal{P}_{\mathrm{fc}}$, the moduli space $M_{W, E}^{*, 0}(p)$ contains no irreducible, rank-one $\mathrm{PU}(2)$ monopoles. The argument of $[16, \S 5.1 .2]$ then allows us to reduce to the case of $C^{\infty}$ parameters. This completes the proof of Theorem 4.1 .

Remark 4.20. It is well-known that a Dirac operator $\mathcal{D}_{A}: \Omega^{0}\left(W^{+} \otimes F\right) \rightarrow$ $\Omega^{0}\left(W^{-} \otimes F\right)$ defined by fixed $\operatorname{spin}^{c}$ connection on $W$ and unitary connection $A$ on a Hermitian vector bundle $F$ over a closed four-manifold has Coker $\mathcal{D}_{A}=0$ when ind $\mathcal{D}_{A} \geq 0$ and the connection $A$ is generic; see, for example, [48, Lemma 6.9.3] for a proof using the Sard-Smale theorem when $F$ is a line bundle. This and related vanishing results for harmonic spinors are proved directly in [4], without appealing to the Sard-Smale theorem.

\section{Appendix A. PU(2) monopoles on Kähler surfaces and $\operatorname{Spin}^{c}$ polynomial invariants.}

\section{A.1. Rank-one, irreducible PU(2) monopoles on Kähler surfaces.}

The observation that the statement and proof of Proposition I.3.5 in [58] is incorrect is an important one due to A. Teleman [54], [71] for the theory of $\operatorname{spin}^{c}$ polynomial invariants and for the developing theory of $\mathrm{PU}(2)$ monopoles. The transversality results given in $[58, \S \mathrm{I}]$, which underly the Donaldson-Pidstrigach-Tyurin theory of $\operatorname{spin}^{c}$ polynomial invariants, rely on [58, Proposition I.3.5]. For completeness, we review Teleman's counterexample here.

We first recall the form of the $\mathrm{PU}(2)$ monopole equations on a Kähler manifold (see [8], [52], [53], [54], [74]). Let $(X, J, g)$ be a four-manifold with almost-complex structure $J$, Hermitian metric $g$, and corresponding Kähler form $\omega$. The canonical $\operatorname{spin}^{c}$ structure is defined by

$$
W_{\text {can }}^{+}:=\Lambda^{0,0} \oplus \Lambda^{0,2}, \quad W_{\text {can }}^{-}:=\Lambda^{0,1},
$$

where $\Lambda^{p, q}=\Lambda^{p, q}\left(T^{*} X\right)$, and $\rho: T^{*} X \rightarrow \operatorname{End}_{\mathbb{C}}(W)$ is the standard Clifford multiplication of [26], [48], [63]. A straightforward modification of Witten's description of the solutions to the $\mathrm{U}(1)$ monopole equations on a Kähler surface [48], [74] then yields the following form of the (unperturbed) $\mathrm{PU}(2)$ 
monopole equations [52], [53] (see also [8]),

$$
\begin{aligned}
F_{A}^{2,0} & =-\frac{1}{2}(\alpha \otimes \bar{\beta})_{0} \\
F_{A}^{0,2} & =\frac{1}{2}(\beta \otimes \bar{\alpha})_{0} \\
i \Lambda_{g} F_{A} & =-\frac{1}{2}\left((\alpha \otimes \bar{\alpha})_{0}-*_{g}(\beta \otimes \bar{\beta})_{0}\right) \\
\bar{\partial}_{A} \alpha+\bar{\partial}_{A}^{*} \beta & =0
\end{aligned}
$$

for a pair $(A, \Phi)$, where $A$ is an $\mathrm{SO}(3)$ connection on $\mathfrak{s u}(E)$, inducing a unitary connection on $E$ via the fixed unitary connection $A_{e}$ on $\operatorname{det} E$, and

$$
\Phi:=(\alpha, \beta) \in \Omega^{0}\left(W_{\text {can }}^{+} \otimes E\right)=\Omega^{0,0}(E) \oplus \Omega^{0,2}(E) .
$$

Now suppose $E$ is a Hermitian two-plane bundle with holomorphic structure $\bar{\partial}_{A}$ over a Kähler surface $X$ with $H^{0}(E) \neq 0$ and that $\alpha$ is a holomorphic section of $E$. If the holomorphic structure $\bar{\partial}_{A}$ on $E$ is indecomposable, then one finds from (A.1) that the triple $(A, \alpha, 0)$ defines an irreducible, rank-one $\mathrm{PU}(2)$ monopole on $X$.

\section{A.2. The definition of $\operatorname{spin}^{c}$ polynomial invariants.}

The basic idea underlying the definition of $\operatorname{spin}^{c}$ polynomial invariants is due to Donaldson [14]. The construction of $\operatorname{spin}^{c}$ polynomial invariants and their development and application to smooth four-manifold topology has been carried out by Pidstrigach and Tyurin in their series of articles [57], [58], [73]. To the best of our knowledge, all results concerning smooth fourmanifold topology which have been proved using $\operatorname{spin}^{c}$ polynomial invariants have been proved independently using either Donaldson invariants or, more recently, Seiberg-Witten invariants. Nonetheless, as the $\operatorname{spin}^{c}$-ASD equations can be viewed as precursor to the $\mathrm{PU}(2)$ monopole equations, the $\operatorname{spin}^{c}$ polynomial invariants at least have some historical interest. The $\operatorname{spin}^{c}-\mathrm{ASD}$ equations can be viewed (the original definition of [58] uses a slightly different trace condition for the unitary connection on $\operatorname{det} E$ ) as the following variant of the $\mathrm{PU}(2)$ monopole equations (1.1):

$$
\begin{aligned}
P_{+}(g) f\left(F_{A}\right) & =0 \\
\left(\mathcal{D}_{A, f}+\rho(f(\vartheta))\right) \Phi & =0 .
\end{aligned}
$$

The moduli space

$$
N_{W, E}(f, g, \vartheta):=\{(A, \Phi): \text { Eq. (A.2) holds }\} /{ }^{\circ} \mathcal{G}_{E}
$$


of $\operatorname{spin}^{c}$-ASD pairs is defined in the same way as the moduli space $M_{W, E}(f, g, \tau, \vartheta)$ of $\mathrm{PU}(2)$ monopoles, with $N_{W, E}^{*}(f, g, \vartheta)$ denoting the space of $\operatorname{spin}^{c}$-ASD pairs $(A, \Phi)$ where is $A$ irreducible. In [58, §I] the moduli space $N_{W, E}^{*}(f, g, \vartheta)$ is used to define invariants of smooth four-manifolds. As we remarked in $\S A .1$, Teleman has pointed out that the proof given by Pidstrigach-Tyurin in [58] that $N_{W, E}^{*}(f, g, \vartheta)$ is a smooth manifold of the expected dimension (see Proposition I.3.5 and Corollaries I.3.6. I.3.7, and I.3.8 in [58]), for generic $(g, \vartheta)$, is incorrect. We note that variations of the Dirac operator $\mathcal{D}_{A, f}+\rho(f(\vartheta))$ with respect to $f \in \Omega^{0}\left(\mathrm{GL}\left(T^{*} X\right)\right)$ are not used in [58], while the one-form $\vartheta$ is assumed to be purely imaginary (a unitary perturbation of the $\mathrm{U}(1)$ connection on $\operatorname{det} W^{+}$), rather than complex as we suppose here. Just as in the proof of our main transversality result, Theorem 1.3, for the moduli space of $\mathrm{PU}(2)$ monopoles, the principal difficulty one needs to address is the possible presence in $N_{W, E}^{*}(f, g, \vartheta)$ of irreducible, rank-one pairs. The proof of Theorem 4.1 carries over, with one slight change, to give:

Theorem A.1. Let $X$ be a closed, oriented, simply-connected,smooth fourmanifold with $C^{\infty}$ Riemannian metric $g$, spin ${ }^{c}$ structure $\left(\rho, W^{+}, W^{-}\right)$, and Hermitian two-plane bundle E. Then there is a first-category subset $\mathcal{P}_{\mathrm{fc}}^{\infty} \subset$ $\mathcal{P}^{\infty}$ such that for all $(f, \vartheta)$ in $\mathcal{P}^{\infty}-\mathcal{P}_{\mathrm{fc}}^{\infty}$ the following holds: The moduli space $N_{W, E}^{*, 0}(f, g, \vartheta)$ contains no spin ${ }^{c}-A S D$ pairs $(A, \Phi)$ with both $A$ irreducible and $\Phi$ rank one.

Proof. The only difference - and this is the reason for the additional constraint on $\pi_{1}(X)$ - is that our unique continuation result for reducible $\mathrm{PU}(2)$ monopoles, Theorem 4.11, must be replaced by the corresponding unique continuation result for reducible anti-self-dual $\mathrm{SO}(3)$ connections [15, Lemma 4.3.21]. The remainder of the proof is otherwise identical to that of Theorem 4.1.

Given Theorem A.1, the remainder of the argument of [58, Proposition I.3.5] yields:

Theorem A.2. Let $X$ be a closed, oriented, simply-connected, smooth fourmanifold with $C^{\infty}$ Riemannian metric $g$, spin ${ }^{c}$ structure $\left(\rho, W^{+}, W^{-}\right)$with spin $^{c}$ connection, and a Hermitian line bundle $\operatorname{det} E$ with unitary connection. Then there is a first-category subset $\mathcal{P}_{\mathrm{fc}}^{\infty}$ of the Fréchet space $\mathcal{P}^{\infty}$ of $C^{\infty}$ perturbation parameters $(f, \vartheta)$ such that for all $(f, \vartheta)$ in $\mathcal{P}^{\infty}-\mathcal{P}_{\mathrm{fc}}^{\infty}$ the 
following holds: For each parameter $(f, \vartheta)$ in $\mathcal{P}^{\infty}-\mathcal{P}_{\mathrm{fc}}^{\infty}$ and Hermitian twoplane bundle $E$ over $X$, the moduli space $N_{W, E}^{*}(f, g, \vartheta)$ of $s p i n^{c}-A S D$ pairs is a smooth manifold of the expected dimension,

$$
\begin{aligned}
\operatorname{dim} N_{W, E}^{*}(f, g, \vartheta)= & -2 p_{1}(\mathfrak{s u}(E))-\frac{3}{2}(e(X)+\sigma(X)) \\
& +\frac{1}{2} p_{1}(\mathfrak{s u}(E))+\frac{1}{2}\left(\left(c_{1}\left(W^{+}\right)+c_{1}(E)\right)^{2}-\sigma(X)\right)-1 .
\end{aligned}
$$

Remark A.3. 1. The holonomy perturbations of [16] can be used to give an alternative, direct proof of Theorem A.2, without addressing the possible presence or absence of irreducible, rank-one spin $^{c}$-ASD pairs in the moduli space $N_{W, E}^{*}(f, g, \vartheta)$.

2. The constraint on the topology of $X$ in the hypotheses of Theorem A.2 can be relaxed by taking account of the possible presence of the 'twisted reducible' anti-self-dual $\mathrm{SO}(3)$ connections of KronheimerMrowka $[38, \S 2]$ with a little more care.

\section{A.3. Some linear algebra.}

When proving Theorem 1.3, our transversality result for the PU(2) monopole equations (1.1), we used the following well-known, elementary fact:

Lemma A.4 ([48, p. 89]). Let $X$ be an oriented, Riemannian, smooth four-manifold with spin ${ }^{c}$ structure $\left(\rho, W^{+}, W^{-}\right)$. Then the Clifford map $\rho: T^{*} X \rightarrow \operatorname{Hom}_{\mathbb{C}}\left(W^{+}, W^{-}\right)$extends to an isomorphism of complex vector bundles,

$$
\rho: T^{*} X \otimes_{\mathbb{R}} \mathbb{C} \rightarrow \operatorname{Hom}_{\mathbb{C}}\left(W^{+}, W^{-}\right) .
$$

The applications arose, specifically, in the proofs of Lemma 2.4 and Proposition 4.10. The lemma is also an essential ingredient in the standard proofs of transversality for the perturbed Seiberg-Witten equations (see, for example, [37], [48], [74]). Hence, though elementary, we include a proof here as we are not aware of a reference.

Proof of Lemma A.4. Suppose $x \in X$. We need to show that the complexlinear map

$$
\rho:\left(T^{*} X\right)_{x} \otimes_{\mathbb{R}} \mathbb{C} \rightarrow \operatorname{Hom}_{\mathbb{C}}\left(W^{+}, W^{-}\right)_{x}
$$

is an isomorphism and, for this purpose, it is very convenient to use the quaternion model for (complex) Clifford multiplication [42], [63]. Thus, we employ the identifications of complex vector spaces, $\left.W^{+}\right|_{x}=\mathbb{H} \oplus 0$ and 
$\left.W^{-}\right|_{x}=0 \oplus \mathbb{H}$. Moreover, with this identification, $\left(T^{*} X\right)_{x}=\mathbb{R}^{4}=\mathbb{H}$ acts on $\left.W\right|_{x}=\mathbb{H} \oplus \mathbb{H}$ by

$$
\rho(v)\left(\phi^{+}, \phi^{-}\right)=\left(v \phi^{-},-\bar{v} \phi^{+}\right), \quad \text { for all } v \in \mathbb{H} \text { and }\left(\phi^{+}, \phi^{-}\right) \in \mathbb{H} \oplus \mathbb{H},
$$

while $i \in \mathbb{C}$ acts by $-I \in \mathbb{H}$ on the right, commuting with Clifford multiplication on the left; we give $\mathbb{H}$ its standard basis $\{1, I, J, K\}$ over $\mathbb{R}$.

Since $\left(T^{*} X\right)_{x} \otimes_{\mathbb{R}} \mathbb{C}$ and $\operatorname{Hom}_{\mathbb{C}}\left(W^{+}, W^{-}\right)_{x}$ both have complex dimension four, it suffices to show that $\rho$ is surjective. Thus, by complex linearity, it is enough to show that for a given unit-norm $\left.\Phi \in W^{+}\right|_{x}$ and $\left.\Psi \in W^{-}\right|_{x}$, there is a $v \in\left(T^{*} X\right)_{x} \otimes_{\mathbb{R}} \mathbb{C}$ such that $\rho(v)=\Psi \otimes\langle\cdot, \Phi\rangle$ or, equivalently, that

$$
\rho(v) \Phi=\Psi .
$$

Suppose we are given $\Phi=\left.(\phi, 0) \in W^{+}\right|_{x}$ and $\Psi=\left.(0, \psi) \in W^{-}\right|_{x}$. Then,

$$
\rho(v) \Phi=v(\phi, 0)=(0,-\bar{v} \phi), \quad \text { for all } v \in \mathbb{H} \text {. }
$$

We want to show that $\rho(v) \Phi=\Psi$ for some $v \in \mathbb{H}$, that is,

$$
(0,-\bar{v} \phi)=(0, \psi) .
$$

But since $\mathbb{H}$ acts transitively on itself by quaternionic multiplication, we can find $v \in \mathbb{H}$ so that (A.4) - and hence (A.3) - holds. This completes the proof.

\section{References.}

[1] R. Abraham, J. E. Marsden, and T. Ratiu, Manifolds, tensor analysis, and applications, second ed., Springer, New York, 1988.

[2] S. Agmon, Unicité et convexité dans les problèmes differentiels, Sém. Math. Sup., 1965, Univ. of Montreal Press, 1966.

[3] S. Agmon and L. Nirenberg, Lower bounds and uniqueness theorems for solutions of differential equations in Hilbert spaces, Comm. Pure Appl. Math. 20 (1967), 207-229.

[4] N. Anghel, Generic vanishing for harmonic spinors of twisted Dirac operators, Proc. Amer. Math. Soc. 124 (1996), 3555-3561.

[5] N. Aronszajn, A unique continuation theorem for solutions of elliptic partial differential equations or inequalities of the second order, J. Math. Pures Appl. 36 (1957), 235-249. 
[6] M. F. Atiyah and I. M. Singer, Index of elliptic operators. IV, Ann. of Math. 93 (1971), 119-138.

[7] J-P. Bourguignon and P. Gauduchon, Spineurs, opérateurs de Dirac et variations de métriques, Comm. Math. Phys. 144 (1992), 581-599.

[8] S. B. Bradlow and O. Garcia-Prada, Non-abelian monopoles and vortices, Proc. Conference on Geometry and Physics (Aarhus, 1995), Lecture Notes in Pure and Appl. Math. 184, Dekker, New York, 1997, pp. 567-589; alg-geom/9602010.

[9] R. Bryant and C. H. Taubes, private communication.

[10] J. B. Conway, A course in functional analysis, Springer, New York, 1985.

[11] S. K. Donaldson, Connections, cohomology and the intersection forms of 4manifolds, J. Differential Geom. 24 (1986), 275-341. The orientation of Yang-Mills moduli spaces and 4-manifold topology, J. Differential Geom. 26 (1987), 397-428.

[13] Polynomial invariants for smooth four-manifolds, Topology $\mathbf{2 9}$ (1990), 257-315. Differential topology and complex variables, Arbeitstagung Proceedings, Max Planck Institut für Mathematik, Bonn, Germany, 1990.

[15] S. K. Donaldson and P. B. Kronheimer, The geometry of four-manifolds, Oxford Univ. Press, Oxford, 1990.

[16] P. M. N. Feehan and T. G. Leness, PU(2) monopoles. I: Regularity, Uhlenbeck compactness, and transversality, J. Differential Geom. 49 (1998), 265-410; $\mathrm{dg}-\mathrm{ga} / 9710032$.

$[17]$ , preprint, 1996 (preliminary version of [16]). $\mathrm{PU}(2)$ monopoles and relations between four-manifold invariants, Topology Appl. 88 (1998), 111-145; dg-ga/9709022. PU(2) monopoles. II: Top-level Seiberg-Witten moduli spaces and Witten's conjecture in low degrees, submitted to a print journal, 1997; $\mathrm{dg}-\mathrm{ga} / 9712005$.

$[20]$ PU(2) monopoles. III: Existence of gluing and obstruction maps, in preparation. tion. , PU(2) monopoles. IV: Surjectivity of gluing maps, in prepara- 
[22] A. Floer, An instanton-invariant for 3-manifolds, Comm. Math. Phys. 118 (1988), 215-240.

[23] D. S. Freed and K. K. Uhlenbeck, Instantons and four-manifolds, second ed., Springer, New York, 1991.

[24] R. Friedman and J. W. Morgan, Smooth four-manifolds and complex surfaces, Springer, Berlin, 1994.

[25] K. Fukaya and K. Ono, Arnold conjecture and Gromov-Witten invariants, Topology 38 (1999), 933-1048.

[26] P. B. Gilkey, Invariance theory, the heat equation, and the Atiyah-Singer index theorem, second ed., Publish or Perish, Wilmington, DE, 1984.

[27] J. Harris, Algebraic geometry, Springer, New York, 1992.

[28] N. Hitchin, Harmonic spinors, Adv. in Math. 14 (1974), 1-55.

[29] Z. Jin and J. L. Kazdan, On the rank of harmonic maps, Math. Z. 207 (1991), 535-537.

[30] R. V. Kadison and J. R. Ringrose, Fundamentals of the theory of operator algebras, Vol. I, Academic Press, New York, 1983.

[31] J. L. Kazdan, Unique continuation in geometry, Comm. Pure Appl. Math. 41 (1988), 667-681.

[32] J. L. Kazdan, Some topics in the study of elliptic equations, Proc. Fourth KIT workshop, Y. H. Choe and U. J. Choi (eds.), Korean Inst. Technology, Math. Research Center, Taeju, Korea, 1989, pp. 41-65.

[33] S. Kobayashi, Differential geometry of complex vector bundles, Princeton Univ. Press, Princeton, NJ, 1987.

[34] U. Koschorke, Infinite-dimensional K-theory and characteristic classes of Fredholm bundle maps, Proc. Symp. Pure Math. 15-1, F. E. Browder (ed.), Amer. Math. Soc., Providence, RI, 1970, pp. 95-133.

[35] D. Kotschick, The Seiberg-Witten equations on symplectic manifolds [after C. H. Taubes], Séminaire Bourbaki 1995/96. Exposés 805-819, Astérisque, vol. 241, Société Mathématique de France, Paris, 1997, Exposé 812, pp. 195220.

[36] P. B. Kronheimer, Embedded surfaces and gauge theory in three and four dimensions, Surveys in Differential Geometry, Vol. III (Cambridge, MA 1996), Internat. Press, Boston, MA, 1998, pp. 243-298. 
[37] P. B. Kronheimer and T. S. Mrowka, The genus of embedded surfaces in the projective plane, Math. Res. Lett. 1 (1994), 797-808.

[38] , Embedded surfaces and the structure of Donaldson's polynomial invariants, J. Differential Geom. 43 (1995), 573-734.

[39] $209-255$.

$[40]$ , Monopoles and contact structures, Invent. Math. 130 (1997),

[41] M. Kuranishi, New proof for the existence of locally complete families of complex structures, Proc. Conference on Complex Analysis, Minneapolis, A. Aeppli, E. Calabi, and H. Röhrl (eds.), Springer, New York, 1965, pp. 142-154.

[42] H. B. Lawson and M-L. Michelsohn, Spin geometry, Princeton Univ. Press, Princeton, NJ, 1988.

[43] J. Li and G. Tian, Virtual moduli cycles and Gromov-Witten invariants of general symplectic manifolds, Topics in sympletic 4-manifolds (Irvine, CA 1996), Internat. Press, Cambridge, MA , 1998, pp. 47-83; alg-geom/9602007.

[44] G. Liu and G. Tian, Arnold conjecture for general symplectic manifolds, J. Differential Geom. 49 (1998), 1-74.

[45] S. Maier, Generic metrics and connections on spin and spin ${ }^{c}$ manifolds, Comm. Math. Phys. 188 (1997), 407-437.

[46] M. Marcolli, Seiberg-Witten gauge theory, Hindustan Book Agency, New Delhi, 1999; Max-Planck-Institut für Mathematik Preprint 1998-85; http://www.mpim-bonn.mpg.de.

[47] G. Moore and E. Witten, Integration over the u-plane in Donaldson theory, Adv. Theor. Math. Phys. 1 (1997), 298-387; hep-th/9709193.

[48] J. W. Morgan, The Seiberg-Witten equations and applications to the topology of smooth four-manifolds, Princeton Univ. Press, Princeton, NJ, 1996.

[49] J. W. Morgan, T. S. Mrowka, and D. Ruberman, $L^{2}$ moduli spaces and a vanishing theorem for Donaldson polynomial invariants, Internat. Press, Cambridge, MA, 1994.

[50] C. B. Morrey, Multiple integrals in the calculus of variations, Springer, New York, 1966.

[51] T. S. Mrowka, P. S. Ozsváth, and B. Yu, Seiberg-Witten monopoles on Seifertfibered spaces, Comm. Anal. Geom. 5 (1997), 685-791; http://www.msri .org. 
[52] C. Okonek and A. Teleman, The coupled Seiberg-Witten equations, vortices, and moduli spaces of stable pairs, Internat. J. Math. 6 (1995), 893-910; alg-geom/9505012.

[53] _ Quaternionic monopoles, Comm. Math. Phys. 180 (1996), 363$388 ;$ alg-geom/9505029.

[54] Recent developments in Seiberg-Witten theory and complex geometry, alg-geom/9612015.

[55] R. S. Palais, Foundations of global non-linear analysis, Benjamin, New York, 1968.

[56] V. Y. Pidstrigach, From Seiberg-Witten to Donaldson: $\mathrm{SO}(3)$ monopole equations, Lecture at the Newton Institute, Cambridge, December 1994.

[57] Some glueing formulas for spin polynomials and a proof of the Van de Ven conjecture, Russian Acad. Sci. Izv. Math. 45 (1995) 529-543.

[58] V. Y. Pidstrigach and A. N. Tyurin, Invariants of the smooth structure of an algebraic surface arising from the Dirac operator, Russian Acad. Sci. Izv. Math. 40 (1993), 267-351.

[59] L L L L Lalization of Donaldson invariants along the Seiberg-Witten classes, dg-ga/9507004.

[60] Y. Ruan, Virtual neighborhoods and pseudoholomorphic curves, alg-geom/9611021.

[61] Y. Ruan and G. Tian, A mathematical theory of quantum cohomology, J. Differential Geom. 42 (1995), 259-367.

[62] W. Rudin, Functional analysis, McGraw-Hill, New York, 1973.

[63] D. Salamon, Spin geometry and Seiberg-Witten invariants, Birkhäuser, Boston, book to appear.

[64] B. Siebert, Gromov-Witten invariants for general symplectic manifolds, alg-geom/9608005.

[65] S. Smale, An infinite-dimensional version of Sard's theorem, Amer. J. Math. 87 (1973), 213-221.

[66] C. H. Taubes, Self-dual connections on 4-manifolds with indefinite intersection matrix, J. Differential Geom. 19 (1984), 517-560.

[67] , preprint, 1993 (preliminary version of [66]).

[68] Casson's invariant and gauge theory, J. Differential Geom. 31 (1990), 547-599. 
[69] $\longrightarrow$ The Seiberg-Witten invariants and symplectic forms, Math. Res. Lett. 1 (1994), 809-822.

[70] A. Teleman, Non-abelian Seiberg-Witten theory and projectively stable pairs, alg-geom/9609020.

[71] —, Moduli spaces of PU(2) monopoles, dg-ga/9702006.

[72] A. Teleman, Untitled preprint.

[73] A. N. Tyurin, Spin-polynomial invariants of smooth structures on algebraic surfaces, Russian Acad. Sci. Izv. Math. 42 (1994) 333-369.

[74] E. Witten, Monopoles and four-manifolds, Math. Res. Lett. 1 (1994), 769-796; hep-th/9411102.

ReCeived August 19, 1998.

Ohio State University

Columbus, $\mathrm{OH} 43210$

E-mail address: feehan@math.ohio-state.edu 